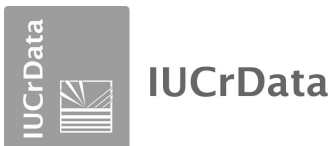

ISSN 2414-3146

Received 28 February 2018

Accepted 1 March 2018

Edited by T. J. Prior, University of Hull, England

Keywords: crystal structure; high-pressure sintering; intermetallic; X-ray diffraction.

CCDC reference: 1826904

Structural data: full structural data are available from iucrdata.iucr.org

\section{Crystal structure of the $\lambda$ - $\mathrm{Al}_{13} \mathrm{Fe}_{4}$-type intermetallic $(\mathrm{Al}, \mathrm{Cu})_{13}(\mathrm{Fe}, \mathrm{Cu})_{4}$}

\author{
Cong Liu and Changzeng Fan*
}

State Key Laboratory of Metastable Materials Science and Technology, Yanshan University, Qinhuangdao 066004, People's Republic of China. *Correspondence e-mail: chzfan@ysu.edu.cn

An $(\mathrm{Al}, \mathrm{Cu})_{13}(\mathrm{Fe}, \mathrm{Cu})_{4}$ phase of [trideca(aluminium/copper) tetra(iron/copper)] was obtained by the high-pressure sintering (HPS) process of a mixture with composition $\mathrm{Al}_{78} \mathrm{Cu}_{15} \mathrm{Fe}_{7}$. Structure analysis suggests that $\mathrm{Cu}$ atoms can replace both $\mathrm{Al}$ and $\mathrm{Fe}$ atoms. Such findings support previous anomalous X-ray scattering (AXS) results although deviate from other reports. The new phase adopts the $\lambda-\mathrm{Al}_{13} \mathrm{Fe}_{4}$ structure type where $\mathrm{Cu}$ atoms partially occupy the $2 c$ and $4 i$ sites for $\mathrm{Al}$ and $\mathrm{Fe}$ atoms, respectively, with occupancies of 0.233 (5) and 0.173 (16).

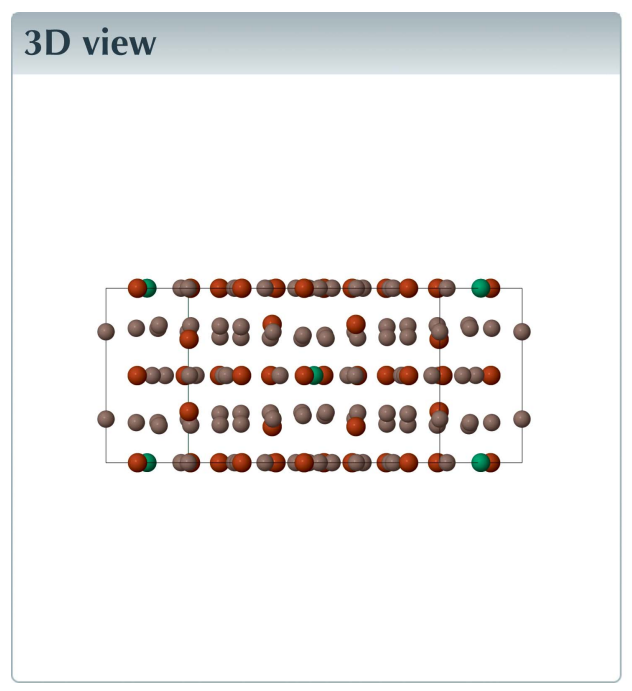

\section{Structure description}

An unnamed $\mathrm{Al}_{78} \mathrm{Cu}_{15} \mathrm{Fe}_{7}$ phase was identified in association with icosahedrite (Ma et al., 2016), the first naturally occurring quasicrystal (Bindi et al., 2009). In simulating its growth mechanism by high pressure sintering (HPS), a $\lambda$ - $\mathrm{Al}_{13} \mathrm{Fe}_{4}$-type phase with composition $\mathrm{Al}_{4.85} \mathrm{Cu}_{0.07} \mathrm{Fe}_{1.46}$ [trideca(aluminium/copper) tetra(iron/copper)] was found. The complex intermetallic compound $\lambda$-phase possesses more than 100 atoms in the unit cell along with $\mathrm{Fe}$ atoms arranged in $\mathrm{Fe}-\mathrm{Al}-\mathrm{Fe}$ groups, which are encapsulated by aluminium atoms (Armbrüster et al., 2012). The synthesized $\mathrm{Al}_{4.85} \mathrm{Cu}_{0.07} \mathrm{Fe}_{1.46}$ phase can also be viewed as a stacking of flat $(F)$ and puckered $(P)$ layers in a $F_{1} P_{1} F_{2} P_{2}$ sequence in the [010] direction (Matilainen et al., 2015). The puckered layers are mirrored against the $F_{2}$ layer. In total, the unit cell contains 102 atoms, where the flat layer is composed of 16 $\mathrm{Al}$ atoms and one commonly occupied $\mathrm{Al} / \mathrm{Cu}$ atom, $7 \mathrm{Fe}$ atoms and one commonly occupied $\mathrm{Fe} / \mathrm{Cu}$ atom. The puckered layer has $22 \mathrm{Al}$ and $4 \mathrm{Fe}$ atoms.

Fig. 1 shows the [010] projection of the crystal structure of the new ternary $\mathrm{Al}-\mathrm{Cu}-\mathrm{Fe}$ phase. The environment of the $\mathrm{Al} / \mathrm{Cu}$ atoms and $\mathrm{Fe} / \mathrm{Cu}$ atoms are shown in Fig. 2. The $\mathrm{Al} /$ $\mathrm{Cu}$ atoms share the $2 c$ site and are connected by $8 \mathrm{Al}$ atoms and $2 \mathrm{Fe}$ atoms. The $\mathrm{Fe} / \mathrm{Cu}$ atoms share the $4 i$ site and are connected by $11 \mathrm{Al}$ atoms. Therefore, the new phase can 

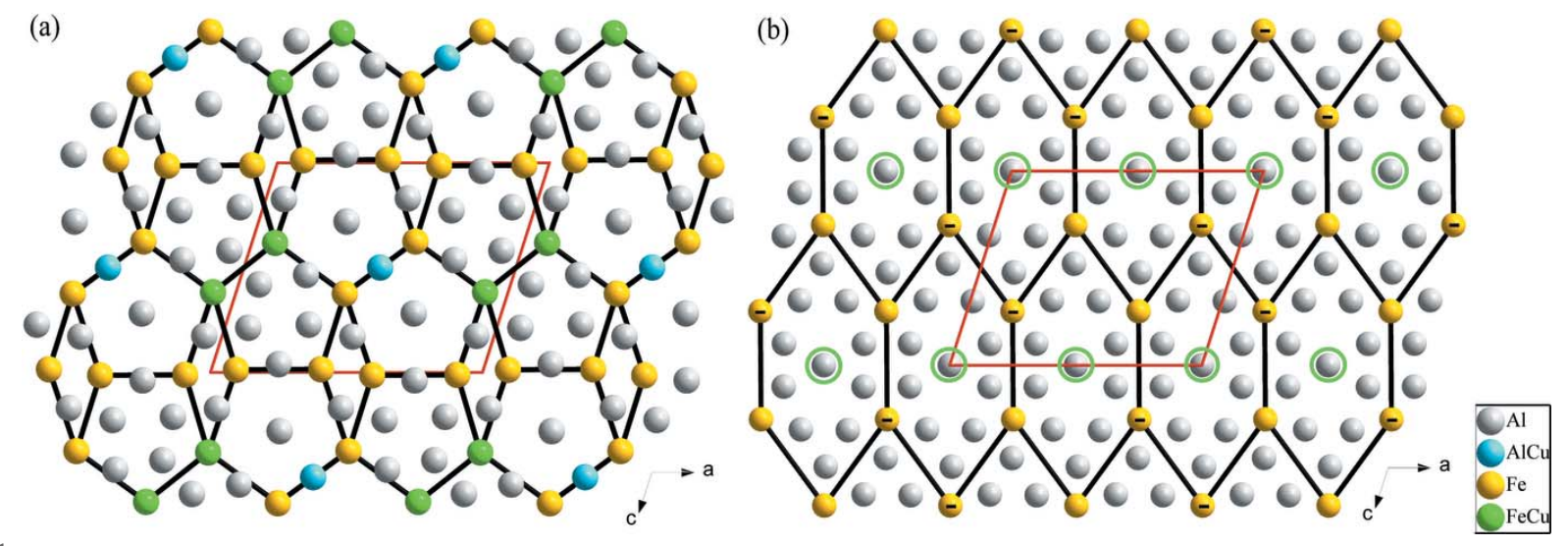

Figure 1

The [010] projection of the crystal structure of the new ternary $\mathrm{Al}-\mathrm{Cu}-\mathrm{Fe}$ phase. The monoclinic unit cell is outlined by a red parallelogram. The tiling formed by $(a)$ connecting transition metal atoms for the flat layer with pentagons, rhombi, and $(b)$ in the case of the puckered layer with elongated hexagons (edge length around $6.5 \AA$ ). The circled $\mathrm{Al}$ atoms refer to the glue atoms and the assigned minus $\mathrm{Fe}$ atoms mean that they are located below the middle plane position.

be described as $(\mathrm{Al}, \mathrm{Cu})_{13}(\mathrm{Fe}, \mathrm{Cu})_{4}$ where $\mathrm{Cu}$ atoms replace both $\mathrm{Al}$ and $\mathrm{Fe}$ atoms, in accordance with the AXS results (Genba et al., 2002) but different from conventional viewpoints (Freiburg \& Grushko, 1994).

\section{Synthesis and crystallization}

The pure elements $\mathrm{Al}$ (indicated purity 99.8\%), $\mathrm{Cu}$ (indicated purity $99.95 \%$ ) and $\mathrm{Fe}$ (indicated purity $99.8 \%$ ) were mixed in the stoichiometric ratio 78:15:7 and initially ground in an agate mortar. Blended powders were then placed in a grinding tool of diameter $5 \mathrm{~mm}$ and pressed into a tablet at about $4 \mathrm{MPa}$ slowly and continuously for about five minutes. A cylindrical block $5 \mathrm{~mm}$ in diameter and $8 \mathrm{~mm}$ in height was obtained without cracks or deformations. The cylindrical block was then inserted in a six-anvil high-pressure apparatus for HPS experiments. Inside the anvil, the block was encapsulated into an $h$-BN crucible covered by an $h$-BN gasket and wrapped in a graphite stove. Both ends of the graphite stove were filled with pyrophyllite and dolomite composite medium and sealed by conductive steel. Finally, all of these components were put into
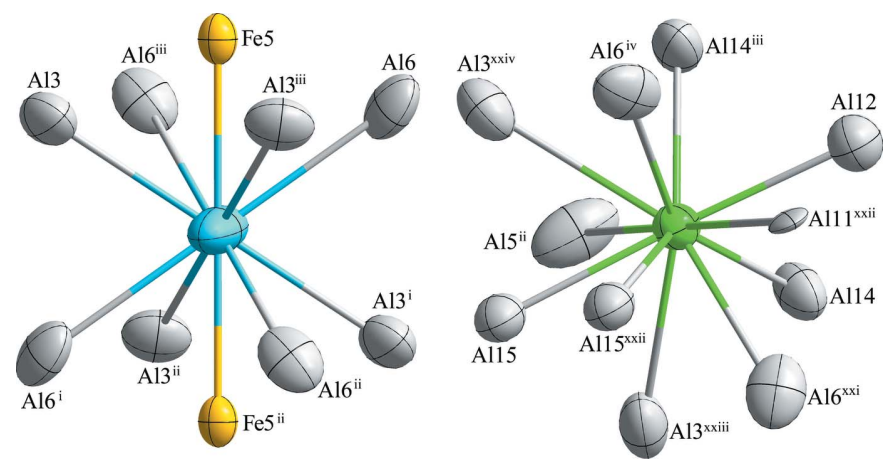

Figure 2

The environment of $\mathrm{Al} / \mathrm{Cu}$ (left) atoms and $\mathrm{Fe} / \mathrm{Cu}$ (right) atoms in detail. Displacement ellipsoids are given at the $99.8 \%$ probability level. [Symmetry codes: (i) $1-x, 1-y, 1-z$; (ii) $1-x, y, 1-z$; (iii) $x$, $1-y, z$; (iv) $\frac{1}{2}+x, \frac{3}{2}-y, z$; (xxi) $-\frac{1}{2}+x, \frac{1}{2}+y, z$; (xxii) $-x, 1-y, 1-z$; (xxiii) $-\frac{1}{2}+x, \frac{3}{2}-y, z ;\left(\right.$ xxiv) $-\frac{1}{2}+x,-\frac{1}{2}+y, z$.] pyrophyllite rock that served as pressure transition medium (see Supporting information). The sample was pressurized up to $5 \mathrm{GPa}$ and heated to $1473 \mathrm{~K}$ for 30 minutes (similiar conditions as employed by Hollister et al., 2014), cooled to $1073 \mathrm{~K}$ and held at this temperature for one hour, and then cooled down rapidly to room temperature. A fragment of dimension $0.08 \times 0.05 \times 0.03 \mathrm{~mm}^{3}$ was selected and mounted on a glass fiber for single-crystal X-ray diffraction measurements.

Table 1

Experimental details.

\begin{tabular}{|c|c|}
\hline \multicolumn{2}{|l|}{ Crystal data } \\
\hline Chemical formula & $\mathrm{Al}_{4.85} \mathrm{Cu}_{0.07} \mathrm{Fe}_{1.46}$ \\
\hline$M_{\mathrm{r}}$ & 216.70 \\
\hline Crystal system, space group & Monoclinic, $C 2 / m$ \\
\hline Temperature $(\mathrm{K})$ & 293 \\
\hline$a, b, c(\AA)$ & $\begin{array}{l}15.4749(10), 8.0913(5) \\
12.4882(9)\end{array}$ \\
\hline$\beta\left(^{\circ}\right)$ & $107.768(2)$ \\
\hline$V\left(\AA^{3}\right)$ & $1489.09(17)$ \\
\hline$Z$ & 16 \\
\hline Radiation type & Мо $K \alpha$ \\
\hline$\mu\left(\mathrm{mm}^{-1}\right)$ & 7.06 \\
\hline Crystal size $(\mathrm{mm})$ & $0.08 \times 0.05 \times 0.03$ \\
\hline \multicolumn{2}{|l|}{ Data collection } \\
\hline Diffractometer & $\begin{array}{l}\text { Bruker D8 Venture Photon } 100 \\
\text { CMOS }\end{array}$ \\
\hline Absorption correction & $\begin{array}{l}\text { Multi-scan (SADABS; Krause et } \\
\quad \text { al., 2015) }\end{array}$ \\
\hline$T_{\min }, T_{\max }$ & $0.576,0.746$ \\
\hline $\begin{array}{l}\text { No. of measured, independent and } \\
\text { observed }[I>2 \sigma(I)] \text { reflections }\end{array}$ & $12161,2319,1749$ \\
\hline$R_{\text {int }}$ & 0.044 \\
\hline$(\sin \theta / \lambda)_{\max }\left(\AA^{-1}\right)$ & 0.715 \\
\hline \multicolumn{2}{|l|}{ Refinement } \\
\hline$R\left[F^{2}>2 \sigma\left(F^{2}\right)\right], w R\left(F^{2}\right), S$ & $0.038,0.070,1.07$ \\
\hline No. of reflections & 2319 \\
\hline No. of parameters & 138 \\
\hline$\Delta \rho_{\max }, \Delta \rho_{\min }\left(\mathrm{e} \AA^{-3}\right)$ & $1.12,-0.99$ \\
\hline
\end{tabular}

Computer programs: APEX3 and SAINT (Bruker, 2015), SHELXT (Sheldrick, 2015a), SHELXL2014 (Sheldrick, 2015b), DIAMOND (Brandenburg \& Putz, 2017) and publCIF (Westrip, 2010). 


\section{Refinement}

Crystal data, data collection and structure refinement details are summarized in Table 1. After considering all atoms in the $\mathrm{Al}_{13} \mathrm{Fe}_{4}$ structure, the original Al1 atom (Wyckoff position 2c) was modelled as occupied with both $\mathrm{Al}$ and $\mathrm{Cu}$ atoms with occupancies of 0.767 (5) and 0.233 (5), respectively. In addition, the $\mathrm{Fe} 2$ position was modelled by partially occupied $\mathrm{Fe}$ atoms [occupancy $0.827(16)$ ] and $\mathrm{Cu}$ atoms [occupancy $0.173(16)]$. Although the refined formula $\mathrm{Al}_{4.85} \mathrm{Cu}_{0.07} \mathrm{Fe}_{1.46}$ shows significant deficiency in $\mathrm{Cu}$ compared to that of initial stoichiometry, it is in good agreement with the EDS measurements.

\section{Acknowledgements}

We greatly acknowledge the financial support from the Hebei Province Youth Top-notch Talent Program (2013-2018).

\section{References}

Armbrüster, M., Kovnir, K., Friedrich, M., Teschner, D., Wowsnick, G., Hahne, M., Gille, P., Szentmiklósi, L., Feuerbacher, M., Heggen, M., Girgsdies, F., Rosenthal, D., Schlögl, R. \& Grin, Y. (2012). Nat. Mater. 11, 690-693.
Bindi, L., Steinhardt, P. J., Yao, N. \& Lu, P. J. (2009). Science, 324, 1306-1309.

Brandenburg, K. \& Putz, H. (2017). DIAMOND. Crystal Impact GbR, Bonn, Germany.

Bruker (2015). APEX3 and SAINT Bruker AXS Inc. Madison, Wisconsin, USA.

Freiburg, C. \& Grushko, B. (1994). J. Alloys Compd. 210, 149-152.

Genba, M., Sugiyama, K., Hiraga, K. \& Yokoyama, Y. (2002). J. Alloys Compd. 342, 143-147.

Hollister, L. S., Bindi, L., Yao, N., Poirier, G. R., Andronicos, C. L., MacPherson, G. J., Lin, C., Distler, V. V., Eddy, M. P., Kostin, A., Kryachko, V., Steinhardt, W. M., Yudovskaya, M., Eiler, J. M., Guan, Y., Clarke, J. J. \& Steinhardt, P. J. (2014). Nat. Commun. 5, 4040-1.

Krause, L., Herbst-Irmer, R., Sheldrick, G. M. \& Stalke, D. (2015). J. Appl. Cryst. 48, 3-10.

Ma, C., Lin, C., Bindi, L. \& Steinhardt, P. J. (2016). LPI Contributions, 1921.

Matilainen, A., Pussi, K., Diehl, R. D., Hahne, M., Gille, P., Gaudry, É., Serkovic Loli, L. N., McGuirk, G. M., de Weerd, M.-C., Fournée, V. \& Ledieu, J. (2015). Phys. Rev. B, 92, 014109.

Sheldrick, G. M. (2015a). Acta Cryst. A71, 3-8.

Sheldrick, G. M. (2015b). Acta Cryst. C71, 3-8.

Westrip, S. P. (2010). J. Appl. Cryst. 43, 920-925. 


\section{full crystallographic data}

IUCrData (2018). 3, x180363 [https://doi.org/10.1107/S2414314618003632]

Crystal structure of the $\lambda$ - $\mathrm{Al}_{13} \mathrm{Fe}_{4}$-type intermetallic $(\mathrm{Al}, \mathrm{Cu})_{13}(\mathrm{Fe}, \mathrm{Cu})_{4}$

\section{Cong Liu and Changzeng Fan}

trideca(aluminium/copper) tetra(iron/copper)

Crystal data

$\mathrm{Al}_{4.85} \mathrm{Cu}_{0.07} \mathrm{Fe}_{1.46}$

$F(000)=1648$

$M_{r}=216.70$

Monoclinic, $C 2 / m$

$a=15.4749(10) \AA$

$b=8.0913(5) \AA$

$c=12.4882(9) \AA$

$\beta=107.768(2)^{\circ}$

$V=1489.09(17) \AA^{3}$

$D_{\mathrm{x}}=3.866 \mathrm{Mg} \mathrm{m}^{-3}$

$Z=16$

Mo $K \alpha$ radiation, $\lambda=0.71073 \AA$

Cell parameters from 1749 reflections

$\theta=2.8-30.5^{\circ}$

$\mu=7.06 \mathrm{~mm}^{-1}$

$T=293 \mathrm{~K}$

Grain, silver

$0.08 \times 0.05 \times 0.03 \mathrm{~mm}$

\section{Data collection}

Bruker D8 Venture Photon 100 CMOS

diffractometer

phi and $\omega$ scans

Absorption correction: multi-scan

(SADABS; Krause et al., 2015)

$T_{\min }=0.576, T_{\max }=0.746$

12161 measured reflections

2319 independent reflections 1749 reflections with $I>2 \sigma(I)$

$R_{\text {int }}=0.044$

$\theta_{\text {max }}=30.5^{\circ}, \theta_{\text {min }}=2.8^{\circ}$

$h=-21 \rightarrow 22$

$k=-11 \rightarrow 10$

$l=-14 \rightarrow 17$

\section{Refinement}

Refinement on $F^{2}$

Least-squares matrix: full

$R\left[F^{2}>2 \sigma\left(F^{2}\right)\right]=0.038$

$w R\left(F^{2}\right)=0.070$

$S=1.07$

2319 reflections

138 parameters

$$
\begin{aligned}
& 0 \text { restraints } \\
& w=1 /\left[\sigma^{2}\left(F_{\mathrm{o}}^{2}\right)+(0.030 P)^{2}+1.0855 P\right] \\
& \quad \text { where } P=\left(F_{\mathrm{o}}^{2}+2 F_{\mathrm{c}}^{2}\right) / 3 \\
& (\Delta / \sigma)_{\max }=0.001 \\
& \Delta \rho_{\max }=1.12 \mathrm{e} \AA^{-3} \\
& \Delta \rho_{\min }=-0.99 \mathrm{e} \AA^{-3}
\end{aligned}
$$

Special details

Geometry. All esds (except the esd in the dihedral angle between two 1.s. planes) are estimated using the full covariance matrix. The cell esds are taken into account individually in the estimation of esds in distances, angles and torsion angles; correlations between esds in cell parameters are only used when they are defined by crystal symmetry. An approximate (isotropic) treatment of cell esds is used for estimating esds involving l.s. planes.

Fractional atomic coordinates and isotropic or equivalent isotropic displacement parameters $\left(\AA^{2}\right)$

\begin{tabular}{llllll}
\hline & $x$ & $y$ & $z$ & $U_{\text {iso }} * U_{\text {eq }}$ & Occ. $(<1)$ \\
\hline Al1 & 0.500000 & 0.500000 & 0.500000 & $0.0098(5)$ & $0.767(5)$ \\
Cu1 & 0.500000 & 0.500000 & 0.500000 & $0.0098(5)$ & $0.233(5)$
\end{tabular}




\begin{tabular}{|c|c|c|c|c|c|}
\hline $\mathrm{A} 12$ & $0.67744(10)$ & 0.500000 & $0.71929(12)$ & $0.0130(3)$ & \\
\hline $\mathrm{Al} 3$ & $0.63633(7)$ & $0.71530(13)$ & $0.52217(8)$ & $0.0092(2)$ & \\
\hline Al4 & $0.80481(10)$ & 1.000000 & $0.77266(12)$ & $0.0077(3)$ & \\
\hline $\mathrm{A} 15$ & $0.76330(10)$ & 0.500000 & $0.46467(13)$ & $0.0126(3)$ & \\
\hline Al6 & $0.49067(7)$ & $0.27023(14)$ & $0.33038(8)$ & $0.0094(2)$ & \\
\hline $\mathrm{Al} 7$ & $0.52074(9)$ & 0.500000 & $0.17059(11)$ & $0.0078(3)$ & \\
\hline $\mathrm{A} 18$ & 0.500000 & $0.74935(18)$ & 0.000000 & $0.0081(3)$ & \\
\hline $\mathrm{A} 19$ & $0.36802(7)$ & $0.71228(13)$ & $0.11008(8)$ & $0.0077(2)$ & \\
\hline Al10 & $0.24072(10)$ & 0.500000 & $-0.03857(13)$ & $0.0118(3)$ & \\
\hline Al11 & $0.08628(9)$ & 0.500000 & $-0.21245(11)$ & $0.0041(3)$ & \\
\hline Al12 & $0.06612(10)$ & 0.500000 & $0.17389(11)$ & $0.0079(3)$ & \\
\hline Al13 & $0.18526(7)$ & $0.71741(13)$ & $0.11114(8)$ & $0.0067(2)$ & \\
\hline Al14 & $0.17782(7)$ & $0.72118(13)$ & $0.33455(8)$ & $0.0081(2)$ & \\
\hline Al15 & $0.07414(9)$ & 0.500000 & $0.58060(11)$ & $0.0068(3)$ & \\
\hline Fe1 & $0.40231(4)$ & 0.500000 & $-0.01441(5)$ & $0.00449(16)$ & \\
\hline $\mathrm{Fe} 2$ & $0.08507(4)$ & 0.500000 & $0.38222(5)$ & $0.0057(2)$ & $0.827(16)$ \\
\hline $\mathrm{Cu} 2$ & $0.08507(4)$ & 0.500000 & $0.38222(5)$ & $0.0057(2)$ & $0.173(16)$ \\
\hline $\mathrm{Fe} 3$ & $0.09041(4)$ & 0.500000 & $-0.01203(5)$ & $0.00434(16)$ & \\
\hline $\mathrm{Fe} 4$ & $0.68011(3)$ & $0.79257(6)$ & $0.72198(4)$ & $0.00551(13)$ & \\
\hline $\mathrm{Fe} 5$ & $0.59919(4)$ & 0.500000 & $0.37535(5)$ & $0.00489(15)$ & \\
\hline
\end{tabular}

Atomic displacement parameters $\left(\AA^{2}\right)$

\begin{tabular}{lllllll}
\hline & $U^{11}$ & $U^{22}$ & $U^{33}$ & $U^{12}$ & $U^{13}$ & $U^{23}$ \\
\hline $\mathrm{A} 11$ & $0.0104(9)$ & $0.0077(9)$ & $0.0144(9)$ & 0.000 & $0.0082(7)$ & 0.000 \\
$\mathrm{Cu} 1$ & $0.0104(9)$ & $0.0077(9)$ & $0.0144(9)$ & 0.000 & $0.0082(7)$ & 0.000 \\
$\mathrm{~A} 12$ & $0.0183(9)$ & $0.0072(8)$ & $0.0132(8)$ & 0.000 & $0.0040(7)$ & 0.000 \\
$\mathrm{~A} 13$ & $0.0100(5)$ & $0.0113(6)$ & $0.0066(5)$ & $-0.0016(5)$ & $0.0031(4)$ & $-0.0020(4)$ \\
$\mathrm{A} 14$ & $0.0066(7)$ & $0.0062(8)$ & $0.0090(7)$ & 0.000 & $0.0006(6)$ & 0.000 \\
$\mathrm{~A} 15$ & $0.0066(7)$ & $0.0088(8)$ & $0.0189(8)$ & 0.000 & $-0.0011(6)$ & 0.000 \\
$\mathrm{~A} 16$ & $0.0068(5)$ & $0.0108(6)$ & $0.0090(5)$ & $-0.0036(4)$ & $0.0001(4)$ & $0.0009(4)$ \\
$\mathrm{A} 17$ & $0.0062(7)$ & $0.0101(8)$ & $0.0059(7)$ & 0.000 & $0.0000(6)$ & 0.000 \\
$\mathrm{~A} 18$ & $0.0070(7)$ & $0.0064(8)$ & $0.0113(7)$ & 0.000 & $0.0031(6)$ & 0.000 \\
$\mathrm{~A} 19$ & $0.0076(5)$ & $0.0093(6)$ & $0.0060(5)$ & $0.0016(4)$ & $0.0016(4)$ & $-0.0008(4)$ \\
$\mathrm{A} 110$ & $0.0048(7)$ & $0.0097(8)$ & $0.0221(8)$ & 0.000 & $0.0061(6)$ & 0.000 \\
$\mathrm{~A} 111$ & $0.0069(7)$ & $0.0008(7)$ & $0.0050(6)$ & 0.000 & $0.0025(5)$ & 0.000 \\
$\mathrm{~A} 112$ & $0.0080(7)$ & $0.0084(8)$ & $0.0074(7)$ & 0.000 & $0.0023(6)$ & 0.000 \\
$\mathrm{~A} 113$ & $0.0059(5)$ & $0.0089(6)$ & $0.0050(5)$ & $-0.0015(4)$ & $0.0011(4)$ & $-0.0013(4)$ \\
$\mathrm{A} 114$ & $0.0097(5)$ & $0.0086(6)$ & $0.0072(5)$ & $-0.0023(4)$ & $0.0044(4)$ & $-0.0010(4)$ \\
$\mathrm{A} 115$ & $0.0078(7)$ & $0.0055(8)$ & $0.0068(7)$ & 0.000 & $0.0018(6)$ & 0.000 \\
$\mathrm{Fe} 1$ & $0.0036(3)$ & $0.0049(4)$ & $0.0050(3)$ & 0.000 & $0.0015(3)$ & 0.000 \\
$\mathrm{Fe} 2$ & $0.0058(4)$ & $0.0055(4)$ & $0.0062(3)$ & 0.000 & $0.0024(3)$ & 0.000 \\
$\mathrm{Cu} 2$ & $0.0058(4)$ & $0.0055(4)$ & $0.0062(3)$ & 0.000 & $0.0024(3)$ & 0.000 \\
$\mathrm{Fe} 3$ & $0.0037(3)$ & $0.0049(4)$ & $0.0044(3)$ & 0.000 & $0.0013(3)$ & 0.000 \\
$\mathrm{Fe} 4$ & $0.0045(2)$ & $0.0067(3)$ & $0.0053(2)$ & $0.0003(2)$ & $0.00140(18)$ & $-0.00024(18)$ \\
$\mathrm{Fe} 5$ & $0.0047(3)$ & $0.0043(4)$ & $0.0047(3)$ & 0.000 & $0.0000(2)$ & 0.000 \\
& & & & & & \\
\hline
\end{tabular}


Geometric parameters $\left(\AA,{ }^{\circ}\right)$

\begin{tabular}{|c|c|c|c|}
\hline $\mathrm{A} 11-\mathrm{Fe} 5$ & $2.4975(7)$ & $\mathrm{Al} 7-\mathrm{Al} 6$ & $2.8672(15)$ \\
\hline $\mathrm{A} 11-\mathrm{Fe} 5^{\mathrm{i}}$ & $2.4976(7)$ & $\mathrm{A} 17-\mathrm{Al} 6^{\mathrm{iii}}$ & $2.8672(15)$ \\
\hline $\mathrm{Al1}-\mathrm{Al} 6$ & $2.7889(11)$ & $\mathrm{A} 17-\mathrm{A} 18^{\mathrm{xiii}}$ & $2.8801(14)$ \\
\hline $\mathrm{A} 11-\mathrm{Al6}^{\mathrm{i}}$ & $2.7889(11)$ & Al7-A18 & $2.8801(14)$ \\
\hline $\mathrm{A} 11-\mathrm{Al}^{6 \mathrm{ii}}$ & $2.7889(11)$ & $\mathrm{A} 18-\mathrm{Fe}^{\mathrm{v}}$ & $2.4942(13)$ \\
\hline $\mathrm{All}-\mathrm{Al} 6^{\mathrm{iii}}$ & $2.7889(11)$ & $\mathrm{A} 18-\mathrm{Fe}^{\mathrm{xiv}}$ & $2.4942(13)$ \\
\hline $\mathrm{Cu} 1-\mathrm{Fe} 5$ & $2.4975(7)$ & $\mathrm{A} 18-\mathrm{Fe}^{\mathrm{xiii}}$ & $2.4944(13)$ \\
\hline $\mathrm{Cu} 1-\mathrm{Fe} 5^{\mathrm{i}}$ & $2.4976(7)$ & $\mathrm{A} 18-\mathrm{Fe} 1$ & $2.4945(13)$ \\
\hline $\mathrm{Cu} 1-\mathrm{Al} 6$ & $2.7889(11)$ & $\mathrm{Al} 8-\mathrm{A} 113^{\mathrm{iv}}$ & $2.7909(10)$ \\
\hline $\mathrm{Cu} 1-\mathrm{Al6}^{\mathrm{i}}$ & $2.7889(11)$ & $\mathrm{Al} 8-\mathrm{Al1} 3^{\mathrm{xiv}}$ & $2.7909(10)$ \\
\hline $\mathrm{Cu} 1-\mathrm{Al}^{6 \mathrm{ii}}$ & $2.7889(11)$ & $\mathrm{Al} 8-\mathrm{A} 19$ & $2.8044(10)$ \\
\hline $\mathrm{Cu} 1-\mathrm{Al}^{\mathrm{iii}}$ & $2.7889(11)$ & $\mathrm{A} 18-\mathrm{A} 19^{\mathrm{xv}}$ & $2.8044(10)$ \\
\hline $\mathrm{A} 12-\mathrm{Fe} 4$ & $2.3677(5)$ & $\mathrm{Al} 8-\mathrm{A} 112^{\mathrm{v}}$ & $2.9188(14)$ \\
\hline $\mathrm{Al} 2-\mathrm{Fe} 4^{\mathrm{iii}}$ & $2.3677(5)$ & $\mathrm{Al} 8-\mathrm{Al} 12^{\mathrm{xiv}}$ & $2.9188(14)$ \\
\hline $\mathrm{A} 12-\mathrm{Al} 3$ & $2.9229(16)$ & $\mathrm{A} 19-\mathrm{Fe} 1$ & $2.4804(11)$ \\
\hline $\mathrm{A} 12-\mathrm{Al} 3^{\mathrm{iii}}$ & $2.9229(16)$ & $\mathrm{A} 19-\mathrm{Fe} 4^{\mathrm{ii}}$ & $2.5157(11)$ \\
\hline $\mathrm{A} 12-\mathrm{A} 19^{\mathrm{ii}}$ & $2.9834(16)$ & $\mathrm{A} 19-\mathrm{A} 111^{\mathrm{xiv}}$ & $2.6462(12)$ \\
\hline $\mathrm{A} 12-\mathrm{A} 19^{\mathrm{i}}$ & $2.9834(16)$ & $\mathrm{A} 19-\mathrm{Al1} 3^{\mathrm{xiv}}$ & $2.6918(14)$ \\
\hline $\mathrm{Al} 3-\mathrm{Fe} 4$ & $2.4585(11)$ & $\mathrm{A} 19-\mathrm{Fe}^{\mathrm{xiv}}$ & $2.7952(11)$ \\
\hline $\mathrm{A} 13-\mathrm{Fe} 5$ & $2.4665(11)$ & A19-Al13 & $2.8327(14)$ \\
\hline $\mathrm{Al} 3-\mathrm{Al1} 4^{\mathrm{iv}}$ & $2.6616(13)$ & Al9-Al10 & $2.8364(16)$ \\
\hline $\mathrm{A} 13-\mathrm{A} 115^{\mathrm{v}}$ & $2.6821(12)$ & $\mathrm{A} 19-\mathrm{Al1} 0^{\mathrm{xiv}}$ & $2.8516(13)$ \\
\hline $\mathrm{Al} 3-\mathrm{Cu} 1$ & $2.6843(10)$ & $\mathrm{A} 110-\mathrm{Fe} 1$ & $2.4257(15)$ \\
\hline $\mathrm{Al} 3-\mathrm{Al} 1$ & $2.6843(10)$ & $\mathrm{Al} 10-\mathrm{Fe} 3$ & $2.4475(15)$ \\
\hline $\mathrm{A} 13-\mathrm{A} 16^{\mathrm{iii}}$ & $2.7452(14)$ & Al10-Al11 & $2.694(2)$ \\
\hline $\mathrm{Al} 3-\mathrm{A} 15^{\mathrm{vi}}$ & $2.7550(13)$ & $\mathrm{Al} 10-\mathrm{Al} 13^{\mathrm{xiv}}$ & $2.8277(13)$ \\
\hline $\mathrm{Al} 3-\mathrm{Fe}^{\mathrm{v}}$ & $2.8538(11)$ & $\mathrm{Al} 10-\mathrm{Al} 13^{\mathrm{xvi}}$ & $2.8277(13)$ \\
\hline $\mathrm{A} 13-\mathrm{A} 15$ & $2.8762(16)$ & $\mathrm{Al} 10-\mathrm{A} 113^{\mathrm{iii}}$ & $2.8820(15)$ \\
\hline $\mathrm{A} 13-\mathrm{A} 114^{\mathrm{ii}}$ & $2.8870(15)$ & Al10-Al13 & $2.8820(15)$ \\
\hline $\mathrm{Al} 4-\mathrm{Fe} 4^{\mathrm{vii}}$ & $2.4891(11)$ & $\mathrm{A} 111-\mathrm{Fe} 3$ & $2.4842(14)$ \\
\hline $\mathrm{A} 14-\mathrm{Fe} 4$ & $2.4891(11)$ & $\mathrm{Al} 11-\mathrm{Fe} 4^{\mathrm{xvii}}$ & $2.5142(11)$ \\
\hline $\mathrm{Al} 4-\mathrm{Al} 7^{\mathrm{vi}}$ & $2.574(2)$ & $\mathrm{Al} 111-\mathrm{Fe} 4^{\mathrm{xviii}}$ & $2.5142(11)$ \\
\hline $\mathrm{A} 14-\mathrm{Fe} 1^{\mathrm{viii}}$ & $2.6265(15)$ & Al11-A115 & $2.5333(19)$ \\
\hline $\mathrm{A} 14-\mathrm{A} 114^{\mathrm{ii}}$ & $2.6785(13)$ & $\mathrm{A} 111-\mathrm{A} 112^{\mathrm{xx}}$ & $2.547(2)$ \\
\hline $\mathrm{A} 14-\mathrm{A} 114^{\mathrm{ix}}$ & $2.6785(13)$ & $\mathrm{A} 111-\mathrm{Fe} 2^{\mathrm{xx}}$ & $2.8452(15)$ \\
\hline $\mathrm{A} 14-\mathrm{Al}^{3} 3^{\mathrm{ix}}$ & $2.6872(12)$ & $\mathrm{Al} 12-\mathrm{Fe} 3$ & $2.4627(15)$ \\
\hline $\mathrm{A} 14-\mathrm{A} 113^{\mathrm{ii}}$ & $2.6872(12)$ & $\mathrm{A} 112-\mathrm{Cu} 2$ & $2.5276(15)$ \\
\hline $\mathrm{A} 14-\mathrm{Fe}^{\mathrm{vi}}$ & $2.7018(16)$ & $\mathrm{Al} 12-\mathrm{Fe} 2$ & $2.5276(15)$ \\
\hline $\mathrm{A} 14-\mathrm{A} 110^{\mathrm{viii}}$ & $2.823(2)$ & $\mathrm{A} 112-\mathrm{Fe}^{\mathrm{xx}}$ & $2.6391(16)$ \\
\hline $\mathrm{Al} 4-\mathrm{Al}^{\mathrm{vi}}$ & $2.827(2)$ & Al12-Al13 & $2.8265(15)$ \\
\hline $\mathrm{Al} 5-\mathrm{Fe} 5$ & $2.4423(16)$ & $\mathrm{Al12}-\mathrm{Al13} 3^{\mathrm{iii}}$ & $2.8265(15)$ \\
\hline $\mathrm{A} 15-\mathrm{Fe} 2^{\mathrm{i}}$ & $2.5344(16)$ & Al12-Al14 & $2.8460(15)$ \\
\hline $\mathrm{A} 15-\mathrm{A} 115^{\mathrm{i}}$ & $2.741(2)$ & $\mathrm{Al12}-\mathrm{Al1} 4^{\mathrm{iii}}$ & $2.8460(15)$ \\
\hline A15-Al14 & $2.8580(14)$ & $\mathrm{A} 113-\mathrm{Fe} 3$ & $2.4974(11)$ \\
\hline Al5-Al14iv & $2.8580(14)$ & $\mathrm{Al} 13-\mathrm{Fe} 4^{\mathrm{ii}}$ & $2.5305(11)$ \\
\hline $\mathrm{Al} 5-\mathrm{Al14} 4^{\mathrm{ii}}$ & $2.9875(16)$ & $\mathrm{Al} 13-\mathrm{Fe}^{\mathrm{xiv}}$ & $2.7431(11)$ \\
\hline
\end{tabular}




\begin{tabular}{|c|c|}
\hline $\mathrm{A} 15-\mathrm{A} 114^{\mathrm{i}}$ & $2.9875(16)$ \\
\hline $\mathrm{Al} 6-\mathrm{Fe} 5$ & $2.4528(11)$ \\
\hline $\mathrm{Al} 6-\mathrm{Fe} 4^{\mathrm{i}}$ & $2.5722(11)$ \\
\hline $\mathrm{A} 16-\mathrm{Fe} 2^{\mathrm{x}}$ & $2.5994(11)$ \\
\hline $\mathrm{A} 16-\mathrm{A} 111^{\mathrm{xi}}$ & $2.7013(13)$ \\
\hline $\mathrm{A} 16-\mathrm{A} 115^{\mathrm{xii}}$ & $2.7755(13)$ \\
\hline $\mathrm{Al} 6-\mathrm{A} 19^{\mathrm{iii}}$ & $2.8292(14)$ \\
\hline $\mathrm{A} 16-\mathrm{A} 114^{\mathrm{x}}$ & $2.9077(15)$ \\
\hline $\mathrm{A} 17-\mathrm{Fe} 1$ & $2.4723(15)$ \\
\hline $\mathrm{A} 17-\mathrm{Fe} 5$ & $2.4734(15)$ \\
\hline $\mathrm{A} 17-\mathrm{Fe} 1^{\mathrm{xiii}}$ & $2.5768(15)$ \\
\hline Al7-A19 & $2.8313(15)$ \\
\hline $\mathrm{A} 17-\mathrm{A} 19^{\mathrm{iii}}$ & $2.8313(15)$ \\
\hline $\mathrm{Fe} 4^{\mathrm{vii}}-\mathrm{A} 14-\mathrm{Fe} 4$ & $84.80(5)$ \\
\hline $\mathrm{Fe}^{\mathrm{vii}}-\mathrm{Al} 4-\mathrm{Al}^{\mathrm{vi}}$ & $137.45(3)$ \\
\hline $\mathrm{Fe} 4-\mathrm{Al} 4-\mathrm{Al} 7^{\mathrm{vi}}$ & $137.45(3)$ \\
\hline $\mathrm{Fe} 4^{\mathrm{vii}}-\mathrm{A} 14-\mathrm{Fe} 1^{\mathrm{viii}}$ & $114.75(4)$ \\
\hline $\mathrm{Fe} 4-\mathrm{Al} 4-\mathrm{Fe}^{\mathrm{viii}}$ & $114.75(4)$ \\
\hline $\mathrm{Al} 7^{\mathrm{vi}}-\mathrm{A} 14-\mathrm{Fe} 1^{\mathrm{viii}}$ & $59.40(5)$ \\
\hline $\mathrm{Fe} 4^{\mathrm{vii}}-\mathrm{Al} 4-\mathrm{Al} 14^{\mathrm{ii}}$ & $128.98(6)$ \\
\hline $\mathrm{Fe} 4-\mathrm{A} 14-\mathrm{Al} 14^{\mathrm{ii}}$ & $59.55(3)$ \\
\hline $\mathrm{Al} 7^{\mathrm{vi}}-\mathrm{A} 14-\mathrm{Al} 14^{\mathrm{ii}}$ & $83.15(4)$ \\
\hline $\mathrm{Fe}^{\mathrm{viii}}-\mathrm{A} 14-\mathrm{Al1} 4^{\mathrm{ii}}$ & $113.07(4)$ \\
\hline $\mathrm{Fe} 4^{\mathrm{vii}}-\mathrm{Al} 4-\mathrm{A} 114^{\mathrm{ix}}$ & $59.55(3)$ \\
\hline $\mathrm{Fe} 4-\mathrm{A} 14-\mathrm{A} 114^{\mathrm{ix}}$ & $128.98(6)$ \\
\hline $\mathrm{A} 17^{\mathrm{vi}}-\mathrm{A} 14-\mathrm{A} 114^{\mathrm{ix}}$ & $83.15(4)$ \\
\hline $\mathrm{Fe}^{\mathrm{viii}}-\mathrm{A} 14-\mathrm{A} 114^{\mathrm{ix}}$ & $113.07(4)$ \\
\hline $\mathrm{A} 114^{\mathrm{ii}}-\mathrm{A} 14-\mathrm{A} 114^{\mathrm{ix}}$ & $114.76(7)$ \\
\hline $\mathrm{Fe} 4^{\mathrm{vii}}-\mathrm{Al} 4-\mathrm{Al} 13^{\mathrm{ix}}$ & $58.38(3)$ \\
\hline $\mathrm{Fe} 4-\mathrm{Al} 4-\mathrm{A} 113^{\mathrm{ix}}$ & $128.55(6)$ \\
\hline $\mathrm{Al} 7^{\mathrm{vi}}-\mathrm{A} 14-\mathrm{A} 113^{\mathrm{ix}}$ & $88.22(4)$ \\
\hline $\mathrm{Fe}^{\mathrm{viii}}-\mathrm{A} 14-\mathrm{A} 113^{\mathrm{ix}}$ & $62.15(3)$ \\
\hline $\mathrm{A} 114^{\mathrm{ii}}-\mathrm{A} 14-\mathrm{A} 113^{\mathrm{ix}}$ & $171.37(7)$ \\
\hline $\mathrm{A} 114^{\mathrm{ix}}-\mathrm{A} 14-\mathrm{A} 113^{\mathrm{ix}}$ & $63.60(3)$ \\
\hline $\mathrm{Fe} 4^{\mathrm{vii}}-\mathrm{Al} 4-\mathrm{Al} 13^{\mathrm{ii}}$ & $128.55(6)$ \\
\hline $\mathrm{Fe} 4-\mathrm{Al} 4-\mathrm{Al} 13^{\mathrm{ii}}$ & $58.38(3)$ \\
\hline $\mathrm{Al} 7^{\mathrm{vi}}-\mathrm{A} 14-\mathrm{A} 113^{\mathrm{ii}}$ & $88.22(4)$ \\
\hline 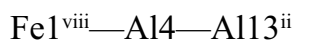 & $62.15(3)$ \\
\hline $\mathrm{A} 114^{\mathrm{ii}}-\mathrm{A} 14-\mathrm{A} 113^{\mathrm{ii}}$ & $63.60(3)$ \\
\hline $\mathrm{A} 114^{\mathrm{ix}}-\mathrm{A} 14-\mathrm{Al} 13^{\mathrm{ii}}$ & $171.37(7)$ \\
\hline $\mathrm{A} 113^{\mathrm{ix}}-\mathrm{A} 14-\mathrm{A} 113^{\mathrm{ii}}$ & $116.62(7)$ \\
\hline $\mathrm{Fe} 4^{\mathrm{vii}}-\mathrm{Al} 4-\mathrm{Fe} 5^{\mathrm{vi}}$ & $111.81(4)$ \\
\hline $\mathrm{Fe} 4-\mathrm{A} 14-\mathrm{Fe}^{\mathrm{vi}}$ & $111.81(4)$ \\
\hline $\mathrm{A} 17^{\mathrm{vi}}-\mathrm{A} 14-\mathrm{Fe}^{\mathrm{vi}}$ & $55.86(4)$ \\
\hline $\mathrm{Fe} 1^{\mathrm{viii}}-\mathrm{A} 14-\mathrm{Fe}^{\mathrm{vi}}$ & $115.26(6)$ \\
\hline $\mathrm{Al} 14^{\mathrm{ii}}-\mathrm{A} 14-\mathrm{Fe}^{\mathrm{vi}}$ & $59.86(3)$ \\
\hline $\mathrm{A} 114^{\mathrm{ix}}-\mathrm{A} 14-\mathrm{Fe}^{\mathrm{vi}}$ & $59.86(3)$ \\
\hline
\end{tabular}

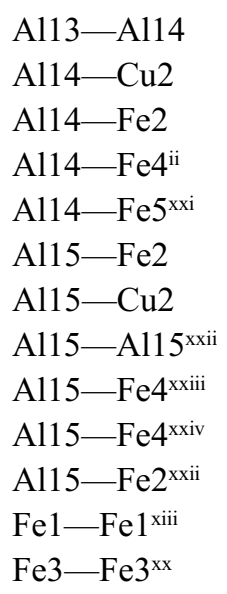

$2.8274(14)$

$2.4776(11)$

$2.4776(11)$

2.5715 (11)

2.6847 (11)

$2.5342(15)$

$2.5342(15)$

2.549 (3)

$2.6185(12)$

$2.6185(12)$

$2.6428(15)$

$2.9337(13)$

$2.9040(13)$

$75.23(5)$

$119.23(4)$

$119.23(4)$

$83.76(5)$

177.35 (7)

$62.50(4)$

$62.50(4)$

$63.26(5)$

136.19 (3)

136.19 (3)

114.09 (7)

65.94 (3)

$58.29(3)$

130.46 (6)

114.74 (4)

92.01 (4)

65.94 (3)

130.46 (6)

58.29 (3)

114.74 (4)

92.01 (4)

123.22 (7)

56.23 (4)

78.70 (4)

78.70 (4)

$126.41(7)$

119.50 (7)

64.54 (3)

64.54 (3)

114.88 (4)

124.41 (6)

58.97 (3)

63.94 (4)

79.83 (4) 


\begin{tabular}{|c|c|}
\hline $\mathrm{Al} 13^{\mathrm{ix}}-\mathrm{A} 14-\mathrm{Fe} 5^{\mathrm{vi}}$ & $114.62(4)$ \\
\hline $\mathrm{Al}^{3} 3^{\mathrm{ii}}-\mathrm{Al} 4-\mathrm{Fe}^{\mathrm{vi}}$ & $114.62(4)$ \\
\hline $\mathrm{Fe} 4^{\mathrm{vii}}-\mathrm{A} 14-\mathrm{A} 110^{\mathrm{viii}}$ & $76.68(4)$ \\
\hline $\mathrm{Fe} 4-\mathrm{Al} 4-\mathrm{Al1} 0^{\mathrm{viii}}$ & $76.68(4)$ \\
\hline $\mathrm{A} 17^{\mathrm{vi}}-\mathrm{A} 14-\mathrm{A} 110^{\mathrm{viii}}$ & $112.11(6)$ \\
\hline $\mathrm{Fe}^{\mathrm{viii}}-\mathrm{A} 14-\mathrm{A} 110^{\mathrm{viii}}$ & $52.72(4)$ \\
\hline $\mathrm{A} 114^{\mathrm{ii}}-\mathrm{A} 14-\mathrm{A} 110^{\mathrm{viii}}$ & $122.14(3)$ \\
\hline $\mathrm{A} 114^{\mathrm{ix}}-\mathrm{A} 14-\mathrm{A} 110^{\mathrm{viii}}$ & $122.14(3)$ \\
\hline $\mathrm{A} 113^{\mathrm{ix}}-\mathrm{A} 14-\mathrm{A} 110^{\text {viii }}$ & $61.70(3)$ \\
\hline $\mathrm{Al}_{13} 3^{\mathrm{ii}}-\mathrm{A} 14-\mathrm{A} 110^{\mathrm{viii}}$ & $61.70(3)$ \\
\hline $\mathrm{Fe}^{\mathrm{vi}}-\mathrm{A} 14-\mathrm{A} 110^{\mathrm{viii}}$ & $167.97(7)$ \\
\hline $\mathrm{Fe}^{\mathrm{vii}}-\mathrm{A} 14-\mathrm{Al}^{\mathrm{vi}}$ & $73.82(4)$ \\
\hline $\mathrm{Fe} 4-\mathrm{Al} 4-\mathrm{Al}^{\mathrm{vi}}$ & $73.82(4)$ \\
\hline $\mathrm{A} 17^{\mathrm{vi}}-\mathrm{A} 14-\mathrm{Al}^{\mathrm{vi}}$ & $108.23(6)$ \\
\hline $\mathrm{Fe}^{\mathrm{viii}}-\mathrm{A} 14-\mathrm{A} 15^{\mathrm{vi}}$ & $167.63(7)$ \\
\hline $\mathrm{A} 114^{\mathrm{ii}}-\mathrm{A} 14-\mathrm{Al} 5^{\mathrm{vi}}$ & $62.48(4)$ \\
\hline $\mathrm{A} 114^{\mathrm{ix}}-\mathrm{A} 14-\mathrm{A} 15^{\mathrm{vi}}$ & $62.48(4)$ \\
\hline $\mathrm{Al} 13^{\mathrm{ix}}-\mathrm{A} 14-\mathrm{A} 15^{\mathrm{vi}}$ & $120.52(3)$ \\
\hline $\mathrm{A} 113^{\mathrm{ii}}-\mathrm{A} 14-\mathrm{A} 15^{\mathrm{vi}}$ & $120.52(3)$ \\
\hline $\mathrm{Fe}^{\mathrm{vi}}-\mathrm{Al} 4-\mathrm{Al} 5^{\mathrm{vi}}$ & $52.38(4)$ \\
\hline $\mathrm{A} 110^{\mathrm{viii}}-\mathrm{A} 14-\mathrm{A} 15^{\mathrm{vi}}$ & $139.65(7)$ \\
\hline $\mathrm{Fe} 4-\mathrm{Al} 2-\mathrm{Fe} 4^{\mathrm{iii}}$ & $177.84(8)$ \\
\hline $\mathrm{Fe} 4-\mathrm{Al} 2-\mathrm{Al} 3$ & $54.16(3)$ \\
\hline 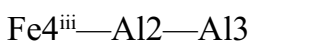 & $127.32(6)$ \\
\hline $\mathrm{Fe} 4-\mathrm{A} 12-\mathrm{Al} 3^{\mathrm{iii}}$ & $127.32(6)$ \\
\hline $\mathrm{Fe} 4^{\mathrm{iii}}-\mathrm{A} 12-\mathrm{A} 13^{\mathrm{iii}}$ & $54.16(3)$ \\
\hline $\mathrm{Al} 3-\mathrm{Al} 2-\mathrm{Al}^{\mathrm{iii}}$ & $73.17(5)$ \\
\hline $\mathrm{Fe} 4-\mathrm{A} 12-\mathrm{A} 19^{\mathrm{ii}}$ & $54.63(3)$ \\
\hline $\mathrm{Fe} 4^{\mathrm{iii}}-\mathrm{A} 12-\mathrm{A} 19^{\mathrm{ii}}$ & $124.91(6)$ \\
\hline $\mathrm{Al} 3-\mathrm{A} 12-\mathrm{Al}^{\mathrm{ii}}$ & $102.72(3)$ \\
\hline $\mathrm{Al} 3^{\mathrm{iii}}-\mathrm{A} 12-\mathrm{A} 19^{\mathrm{ii}}$ & $155.03(7)$ \\
\hline $\mathrm{Fe} 4-\mathrm{A} 12-\mathrm{A} 19^{\mathrm{i}}$ & $124.91(6)$ \\
\hline 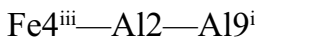 & $54.63(3)$ \\
\hline $\mathrm{A} 13-\mathrm{A} 12-\mathrm{A} 19^{\mathrm{i}}$ & $155.02(7)$ \\
\hline $\mathrm{A} 13^{\mathrm{iii}}-\mathrm{A} 12-\mathrm{A} 19^{\mathrm{i}}$ & $102.72(3)$ \\
\hline $\mathrm{A} 19^{\mathrm{ii}}-\mathrm{A} 12-\mathrm{A} 19^{\mathrm{i}}$ & $70.30(5)$ \\
\hline $\mathrm{Fe} 4-\mathrm{Al} 3-\mathrm{Fe} 5$ & $149.72(5)$ \\
\hline $\mathrm{Fe} 4-\mathrm{Al} 3-\mathrm{Al14} 4^{\mathrm{iv}}$ & $141.13(5)$ \\
\hline $\mathrm{Fe} 5-\mathrm{Al} 3-\mathrm{All}^{\mathrm{iv}}$ & $63.00(3)$ \\
\hline $\mathrm{Fe} 4-\mathrm{Al} 3-\mathrm{Al}_{15} 5^{\mathrm{V}}$ & $61.06(4)$ \\
\hline $\mathrm{Fe} 5-\mathrm{Al} 3-\mathrm{Al} 15^{\mathrm{v}}$ & $141.94(5)$ \\
\hline $\mathrm{A} 114^{\mathrm{iv}}-\mathrm{Al} 3-\mathrm{A} 115^{\mathrm{v}}$ & $106.06(5)$ \\
\hline $\mathrm{Fe} 4-\mathrm{Al} 3-\mathrm{Cu} 1$ & $103.55(4)$ \\
\hline $\mathrm{Fe} 5-\mathrm{Al} 3-\mathrm{Cu} 1$ & $57.82(3)$ \\
\hline $\mathrm{Al} 14^{\mathrm{iv}}-\mathrm{Al} 3-\mathrm{Cu} 1$ & $115.32(4)$ \\
\hline $\mathrm{A} 115^{\mathrm{v}}-\mathrm{A} 13-\mathrm{Cu} 1$ & $104.45(4)$ \\
\hline $\mathrm{Fe} 4-\mathrm{Al} 3-\mathrm{Al} 1$ & $103.55(4)$ \\
\hline $\mathrm{Fe} 5-\mathrm{Al} 3-\mathrm{Al} 1$ & $57.82(3)$ \\
\hline
\end{tabular}

\begin{tabular}{|c|c|}
\hline $\mathrm{A} 19^{\mathrm{xiv}}-\mathrm{A} 111-\mathrm{Al}^{6 \mathrm{xi}}$ & $169.68(6)$ \\
\hline $\mathrm{A} 19^{\mathrm{xvi}}-\mathrm{A} 111-\mathrm{Al} 6^{\mathrm{xi}}$ & $63.87(3)$ \\
\hline $\mathrm{A} 110-\mathrm{A} 111-\mathrm{Al}^{\mathrm{xi}}$ & $125.06(3)$ \\
\hline $\mathrm{Fe} 3-\mathrm{A} 111-\mathrm{A} 16^{\mathrm{xxvi}}$ & $114.88(4)$ \\
\hline $\mathrm{Fe} 4^{\mathrm{xvii}}-\mathrm{A} 111-\mathrm{Al} 6^{\mathrm{xxvi}}$ & $58.97(3)$ \\
\hline $\mathrm{Fe} 4^{\mathrm{xviii}}-\mathrm{A} 111-\mathrm{Al} 6^{\mathrm{xxvi}}$ & $124.41(6)$ \\
\hline $\mathrm{A} 115^{\mathrm{xix}}-\mathrm{A} 111-\mathrm{A} 16^{\mathrm{xxvi}}$ & $63.95(4)$ \\
\hline $\mathrm{A} 112^{\mathrm{xx}}-\mathrm{A} 111-\mathrm{A} 16^{\mathrm{xxvi}}$ & $79.83(4)$ \\
\hline $\mathrm{A} 19^{\mathrm{xiv}}-\mathrm{A} 111-\mathrm{A} 16^{\mathrm{xxvi}}$ & $63.87(3)$ \\
\hline $\mathrm{A} 19^{\mathrm{xvi}}-\mathrm{A} 111-\mathrm{A} 16^{\mathrm{xxvi}}$ & $169.68(6)$ \\
\hline $\mathrm{A} 110-\mathrm{A} 111-\mathrm{A} 16^{\mathrm{xxvi}}$ & $125.06(3)$ \\
\hline $\mathrm{A} 16^{\mathrm{xi}}-\mathrm{A} 111-\mathrm{A} 16^{\mathrm{xxvi}}$ & $108.08(6)$ \\
\hline $\mathrm{Fe} 3-\mathrm{Al} 11-\mathrm{Fe}^{\mathrm{xx}}$ & $118.84(5)$ \\
\hline $\mathrm{Fe} 4^{\mathrm{xvii}}-\mathrm{A} 111-\mathrm{Fe}^{\mathrm{xx}}$ & $104.89(4)$ \\
\hline 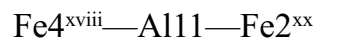 & $104.89(4)$ \\
\hline $\mathrm{A} 115^{\mathrm{xix}}-\mathrm{A} 111-\mathrm{Fe} 2^{\mathrm{xx}}$ & $58.52(4)$ \\
\hline $\mathrm{A} 112^{\mathrm{xx}}-\mathrm{A} 111-\mathrm{Fe} 2^{\mathrm{xx}}$ & $55.57(4)$ \\
\hline $\mathrm{A} 19^{\mathrm{xiv}}-\mathrm{A} 111-\mathrm{Fe} 2^{\mathrm{xx}}$ & $114.26(4)$ \\
\hline $\mathrm{A} 19^{\mathrm{xvi}}-\mathrm{A} 111-\mathrm{Fe} 2^{\mathrm{xx}}$ & $114.26(4)$ \\
\hline $\mathrm{A} 110-\mathrm{A} 111-\mathrm{Fe} 2^{\mathrm{xx}}$ & $175.07(6)$ \\
\hline $\mathrm{A} 16^{\mathrm{xi}}-\mathrm{A} 111-\mathrm{Fe}^{\mathrm{xx}}$ & $55.82(3)$ \\
\hline $\mathrm{A} 16^{\mathrm{xxvi}}-\mathrm{A} 111-\mathrm{Fe}^{\mathrm{xx}}$ & $55.82(3)$ \\
\hline $\mathrm{Fe} 3-\mathrm{Al12}-\mathrm{Cu} 2$ & $165.30(7)$ \\
\hline $\mathrm{Fe} 3-\mathrm{A} 112-\mathrm{Fe} 2$ & $165.30(7)$ \\
\hline $\mathrm{Fe} 3-\mathrm{A} 112-\mathrm{A} 111^{\mathrm{xx}}$ & $126.50(7)$ \\
\hline $\mathrm{Fe} 2-\mathrm{A} 112-\mathrm{A} 111^{\mathrm{xx}}$ & $68.20(5)$ \\
\hline $\mathrm{Fe} 3-\mathrm{Al} 12-\mathrm{Fe}^{\mathrm{xx}}$ & $69.29(4)$ \\
\hline $\mathrm{Fe} 2-\mathrm{A} 112-\mathrm{Fe}^{\mathrm{xx}}$ & $125.41(6)$ \\
\hline $\mathrm{A} 111^{\mathrm{xx}}-\mathrm{A} 112-\mathrm{Fe} 3^{\mathrm{xx}}$ & $57.21(5)$ \\
\hline $\mathrm{Fe} 3-\mathrm{A} 112-\mathrm{A} 113$ & $55.84(3)$ \\
\hline $\mathrm{Cu} 2-\mathrm{Al} 12-\mathrm{A} 113$ & $113.87(5)$ \\
\hline $\mathrm{Fe} 2-\mathrm{A} 112-\mathrm{Al13}$ & $113.87(5)$ \\
\hline $\mathrm{A} 111^{\mathrm{xx}}-\mathrm{A} 112-\mathrm{A} 113$ & $140.57(3)$ \\
\hline $\mathrm{Fe}^{\mathrm{xx}}-\mathrm{A} 112-\mathrm{A} 113$ & $108.15(5)$ \\
\hline $\mathrm{Fe} 3-\mathrm{A} 112-\mathrm{A} 113^{\mathrm{iii}}$ & $55.84(3)$ \\
\hline $\mathrm{Fe} 2-\mathrm{A} 112-\mathrm{A} 113^{\mathrm{iii}}$ & $113.87(5)$ \\
\hline $\mathrm{A} 111^{\mathrm{xx}}-\mathrm{A} 112-\mathrm{A} 113^{\mathrm{iii}}$ & $140.57(3)$ \\
\hline $\mathrm{Fe} 3^{\mathrm{xx}}-\mathrm{A} 112-\mathrm{A} 113^{\mathrm{iii}}$ & $108.15(5)$ \\
\hline $\mathrm{Al13}-\mathrm{A} 112-\mathrm{Al} 13^{\mathrm{iii}}$ & $76.98(5)$ \\
\hline $\mathrm{Fe} 3-\mathrm{Al12}-\mathrm{Al14}$ & $115.47(5)$ \\
\hline $\mathrm{Cu} 2-\mathrm{Al} 12-\mathrm{Al} 14$ & $54.52(3)$ \\
\hline $\mathrm{Fe} 2-\mathrm{Al12}-\mathrm{Al} 14$ & $54.52(3)$ \\
\hline $\mathrm{A} 111^{\mathrm{xx}}-\mathrm{A} 112-\mathrm{A} 114$ & $105.36(5)$ \\
\hline $\mathrm{Fe}^{\mathrm{xx}}-\mathrm{A} 112-\mathrm{Al} 14$ & $139.29(3)$ \\
\hline Al13-Al12-Al14 & $59.79(4)$ \\
\hline $\mathrm{A} 113^{\mathrm{iii}-\mathrm{A} 112-\mathrm{A} 114}$ & $106.24(6)$ \\
\hline $\mathrm{Fe} 3-\mathrm{Al12}-\mathrm{Al1} 4^{\mathrm{iii}}$ & $115.47(5)$ \\
\hline $\mathrm{Fe} 2-\mathrm{Al} 12-\mathrm{Al14} 4^{\mathrm{iii}}$ & $54.52(3)$ \\
\hline
\end{tabular}




\begin{tabular}{|c|c|}
\hline Al14证-Al3-Al1 & $115.32(4)$ \\
\hline $\mathrm{A} 115^{\mathrm{v}}-\mathrm{A} 13-\mathrm{A} 11$ & $104.45(4)$ \\
\hline $\mathrm{Fe} 4-\mathrm{Al} 3-\mathrm{Al} 6^{\mathrm{iii}}$ & $140.65(5)$ \\
\hline $\mathrm{Fe} 5-\mathrm{Al} 3-\mathrm{Al} 6^{\mathrm{iii}}$ & $55.84(3)$ \\
\hline $\mathrm{Al} 14^{\mathrm{iv}}-\mathrm{Al} 3-\mathrm{Al} 6^{\mathrm{iii}}$ & $65.04(4)$ \\
\hline $\mathrm{A} 115^{\mathrm{v}}-\mathrm{Al} 3-\mathrm{Al} 6^{\mathrm{iii}}$ & $86.19(5)$ \\
\hline $\mathrm{A} 11-\mathrm{Al} 3-\mathrm{A} 16^{\mathrm{iii}}$ & $61.80(3)$ \\
\hline $\mathrm{Fe} 4-\mathrm{Al} 3-\mathrm{A} 15^{\mathrm{vi}}$ & $75.59(4)$ \\
\hline $\mathrm{Fe} 5-\mathrm{Al} 3-\mathrm{A} 15^{\mathrm{vi}}$ & $129.35(5)$ \\
\hline $\mathrm{A} 114^{\mathrm{iv}}-\mathrm{Al} 3-\mathrm{A} 15^{\mathrm{vi}}$ & $66.92(5)$ \\
\hline $\mathrm{A} 115^{\mathrm{v}}-\mathrm{Al} 3-\mathrm{Al} 5^{\mathrm{vi}}$ & $60.54(4)$ \\
\hline $\mathrm{A} 11-\mathrm{A} 13-\mathrm{A} 15^{\mathrm{vi}}$ & $163.70(5)$ \\
\hline $\mathrm{Al} 6^{\mathrm{iii}}-\mathrm{Al} 3-\mathrm{Al} 5^{\mathrm{vi}}$ & $108.31(5)$ \\
\hline $\mathrm{Fe} 4-\mathrm{A} 13-\mathrm{Fe} 2^{\mathrm{v}}$ & $110.81(4)$ \\
\hline $\mathrm{Fe} 5-\mathrm{Al} 3-\mathrm{Fe} 2^{\mathrm{v}}$ & $99.01(4)$ \\
\hline $\mathrm{Al} 14^{\mathrm{iv}}-\mathrm{Al} 3-\mathrm{Fe} 2^{\mathrm{v}}$ & $53.25(3)$ \\
\hline $\mathrm{A} 115^{\mathrm{v}}-\mathrm{A} 13-\mathrm{Fe} 2^{\mathrm{v}}$ & $54.38(4)$ \\
\hline $\mathrm{A} 11-\mathrm{Al} 3-\mathrm{Fe}^{\mathrm{v}}$ & $113.45(4)$ \\
\hline $\mathrm{A} 16^{\mathrm{iii}}-\mathrm{A} 13-\mathrm{Fe}^{\mathrm{v}}$ & $55.28(3)$ \\
\hline $\mathrm{A} 15^{\mathrm{vi}}-\mathrm{Al} 3-\mathrm{Fe}^{\mathrm{v}}$ & $53.69(4)$ \\
\hline $\mathrm{Fe} 4-\mathrm{Al} 3-\mathrm{Al} 5$ & $114.19(5)$ \\
\hline $\mathrm{Fe} 5-\mathrm{Al} 3-\mathrm{Al} 5$ & $53.74(4)$ \\
\hline $\mathrm{Al} 14^{\mathrm{iv}}-\mathrm{Al} 3-\mathrm{Al} 5$ & $62.00(4)$ \\
\hline $\mathrm{A} 115^{\mathrm{v}}-\mathrm{A} 13-\mathrm{A} 15$ & $156.54(5)$ \\
\hline $\mathrm{Cu} 1-\mathrm{Al} 3-\mathrm{A} 15$ & $99.00(4)$ \\
\hline $\mathrm{A} 11-\mathrm{Al3}-\mathrm{A} 15$ & $99.00(4)$ \\
\hline $\mathrm{Al} 6^{\mathrm{iii}}-\mathrm{A} 13-\mathrm{A} 15$ & $104.45(5)$ \\
\hline $\mathrm{A} 15^{\mathrm{vi}}-\mathrm{Al} 3-\mathrm{A} 15$ & $96.06(3)$ \\
\hline $\mathrm{Fe} 2^{\mathrm{v}}-\mathrm{A} 13-\mathrm{A} 15$ & $114.79(4)$ \\
\hline $\mathrm{Fe} 4-\mathrm{Al} 3-\mathrm{Al} 14^{\mathrm{ii}}$ & $56.83(3)$ \\
\hline $\mathrm{Fe} 5-\mathrm{Al} 3-\mathrm{A} 114^{\mathrm{ii}}$ & $115.75(5)$ \\
\hline $\mathrm{A} 114^{\mathrm{iv}}-\mathrm{A} 13-\mathrm{A} 114^{\mathrm{ii}}$ & $94.59(4)$ \\
\hline $\mathrm{Al1} 15^{\mathrm{v}}-\mathrm{A} 13-\mathrm{A} 114^{\mathrm{ii}}$ & $100.92(5)$ \\
\hline $\mathrm{Al} 1-\mathrm{Al} 3-\mathrm{Al} 14^{\mathrm{ii}}$ & $132.65(4)$ \\
\hline $\mathrm{Al}^{\mathrm{iii}}-\mathrm{A} 13-\mathrm{A} 114^{\mathrm{ii}}$ & $159.63(5)$ \\
\hline $\mathrm{Al} 5^{\mathrm{vi}}-\mathrm{Al} 3-\mathrm{A} 114^{\mathrm{ii}}$ & $60.82(4)$ \\
\hline $\mathrm{Fe} 2^{\mathrm{v}}-\mathrm{Al} 3-\mathrm{A} 114^{\mathrm{ii}}$ & $113.85(4)$ \\
\hline $\mathrm{Al} 5-\mathrm{Al} 3-\mathrm{Al} 14^{\mathrm{ii}}$ & $62.45(4)$ \\
\hline $\mathrm{Fe} 4-\mathrm{Al} 3-\mathrm{A} 12$ & $51.32(3)$ \\
\hline $\mathrm{Fe} 5-\mathrm{Al} 3-\mathrm{Al} 2$ & $98.43(4)$ \\
\hline $\mathrm{A} 114^{\mathrm{iv}}-\mathrm{A} 13-\mathrm{A} 12$ & $143.85(5)$ \\
\hline $\mathrm{A} 115^{\mathrm{v}}-\mathrm{A} 13-\mathrm{A} 12$ & $106.66(5)$ \\
\hline $\mathrm{Cu} 1-\mathrm{Al} 3-\mathrm{Al} 2$ & $70.26(4)$ \\
\hline $\mathrm{A} 11-\mathrm{A} 13-\mathrm{Al} 2$ & $70.26(4)$ \\
\hline $\mathrm{Al} 6^{\mathrm{iii}}-\mathrm{Al} 3-\mathrm{A} 12$ & $132.07(5)$ \\
\hline $\mathrm{A} 15^{\mathrm{vi}}-\mathrm{Al} 3-\mathrm{A} 12$ & $118.31(5)$ \\
\hline $\mathrm{Fe} 2^{\mathrm{v}}-\mathrm{Al} 3-\mathrm{Al} 2$ & $160.96(5)$ \\
\hline $\mathrm{A} 15-\mathrm{A} 13-\mathrm{A} 12$ & $81.93(5)$ \\
\hline
\end{tabular}

Al11 ${ }^{\mathrm{xx}}$-Al12-Al14 $4^{\mathrm{iii}} \quad 105.36$ (5)

$\mathrm{Fe}^{\mathrm{xx}}$-Al12-Al14 $4^{\mathrm{iii}} \quad 139.29$ (3)

Al13-Al12-Al14 ${ }^{\mathrm{iii}} \quad 106.24$ (6)

Al13 $3^{\text {iii_-Al12-Al14 }}{ }^{\text {iii }} \quad 59.79$ (3)

Al14-Al12-Al14 iii 77.93 (6)

$\mathrm{Fe} 3$-Al12-A18 ${ }^{\text {xiv }} \quad 54.43$ (3)

$\mathrm{Fe} 2-\mathrm{A} 112-\mathrm{A} 18^{\mathrm{xxiv}} \quad 132.07$ (3)

Al11 $1^{\mathrm{xx}}$-Al12-Al8 ${ }^{\mathrm{xxiv}} \quad 90.35$ (4)

$\mathrm{Fe}^{\mathrm{xx}}-\mathrm{A} 112-\mathrm{Al} 8^{\mathrm{xxiv}} \quad 53.04$ (3)

Al13-Al12-A18 ${ }^{\text {xxiv }} \quad 109.66(5)$

Al13 $3^{\mathrm{iii}}$-A112-A18 ${ }^{\text {xiv }} \quad 58.10$ (3)

Al14-A112-A18 ${ }^{\text {xxiv }} \quad 163.95$ (6)

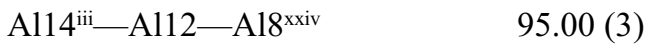

$\mathrm{Fe} 3$-Al12-Al8 ${ }^{\text {xiv }} \quad 54.43$ (3)

$\mathrm{Fe} 2-\mathrm{A} 112-\mathrm{A} 18^{\mathrm{xiv}}$

Al11 ${ }^{\mathrm{xx}}-\mathrm{A} 112-\mathrm{A} 18^{\mathrm{xiv}} \quad 90.35$ (4)

$\mathrm{Fe}^{\mathrm{xx}}-\mathrm{Al} 12-\mathrm{A} 18^{\mathrm{xiv}} \quad 53.04$ (3)

Al13-Al12-Al8 ${ }^{\text {xiv }} \quad 58.10$ (3)

Al13 $3^{\mathrm{iii}}-\mathrm{A} 112-\mathrm{A} 18^{\mathrm{xiv}} \quad 109.66(5)$

Al14-Al12-Al8 ${ }^{\text {xiv }} \quad 95.00$ (3)

Al14 ${ }^{\mathrm{iii}-\mathrm{A}} \mathrm{A} 112-\mathrm{A} 18^{\mathrm{xiv}} \quad 163.95(6)$

Al8 ${ }^{\text {xxiv }}-\mathrm{A} 112-\mathrm{A} 18^{\text {xiv }} \quad 88.03$ (6)

$\mathrm{Fe} 3-\mathrm{Al13}-\mathrm{Fe} 4^{\mathrm{ii}} \quad 149.09$ (5)

$\mathrm{Fe} 3-\mathrm{Al13}$-A14 ${ }^{\mathrm{ix}} \quad 149.09$ (5)

$\mathrm{Fe} 4^{\mathrm{ii}}$-Al13-Al4 ${ }^{\mathrm{ix}} \quad 56.89$ (4)

Fe3-Al13-A19 ${ }^{\text {xiv }} \quad 65.06$ (3)

$\mathrm{Fe}^{\mathrm{ii}}-\mathrm{A} 113-\mathrm{A} 19^{\mathrm{xiv}} \quad 135.38(5)$

Al4 ${ }^{\text {ix }}$-Al13-A19 $9^{\text {xiv }} \quad 108.87$ (5)

$\mathrm{Fe} 3-\mathrm{Al13}-\mathrm{Fe}^{\mathrm{xiv}} \quad 101.39$ (4)

$\mathrm{Fe}^{\mathrm{ii}}-\mathrm{A} 113-\mathrm{Fe}^{\mathrm{xiv}} \quad 109.53$ (4)

$\mathrm{Al} 4^{\mathrm{ix}}-\mathrm{A} 113-\mathrm{Fe}^{\mathrm{xiv}} \quad 57.84$ (4)

$\mathrm{A} 19^{\mathrm{xiv}}-\mathrm{A} 113-\mathrm{Fe}^{\mathrm{xiv}} \quad 54.30$ (3)

$\mathrm{Fe} 3$-Al13-Al8 ${ }^{\mathrm{xiv}} \quad 55.95$ (4)

$\mathrm{Fe} 4^{\mathrm{ii}}-\mathrm{Al13}-\mathrm{Al} 8^{\mathrm{xiv}} \quad 148.11(5)$

$\mathrm{A} 14^{\mathrm{ix}}$-Al13-A18 $8^{\mathrm{xiv}} \quad 93.86$ (5)

$\mathrm{A} 19^{\mathrm{xiv}}-\mathrm{A} 113-\mathrm{A} 18^{\mathrm{xiv}} \quad 61.50(3)$

$\mathrm{Fe}^{\text {xiv }}$-Al13-Al8 $8^{\text {xiv }} \quad 53.57$ (4)

$\mathrm{Fe} 3$-Al13-Al12 54.68 (3)

$\mathrm{Fe}^{\mathrm{ii}}$-Al13-Al12 111.35 (5)

Al4 $4^{\mathrm{ix}}$-Al13-Al12 108.33 (5)

$\mathrm{A} 19^{\mathrm{xiv}}$-Al13-Al12 113.25 (5)

$\mathrm{Fe}^{\mathrm{xiv}}$-Al13-Al12 111.38 (4)

Al8 ${ }^{\mathrm{xiv}}$-Al13-Al12 62.61 (4)

$\mathrm{Fe} 3$-Al13-Al14 114.97 (4)

$\mathrm{Fe} 4$ ii-Al13-Al14 57.04 (3)

Al4 $4^{\mathrm{ix}}$-Al13-Al14 58.05 (4)

A19 ${ }^{\text {xiv }}$-Al13-Al14 $156.71(5)$

$\mathrm{Fe}^{\mathrm{xiv}}$-Al13-Al14 105.20 (4) 


\begin{tabular}{|c|c|}
\hline $\mathrm{A} 114^{\mathrm{ii}}-\mathrm{A} 13-\mathrm{A} 12$ & $64.42(4)$ \\
\hline $\mathrm{Fe} 5-\mathrm{A} 15-\mathrm{Fe}^{\mathrm{i}}$ & $159.85(7)$ \\
\hline $\mathrm{Fe} 5-\mathrm{Al} 5-\mathrm{A} 115^{\mathrm{i}}$ & $142.89(7)$ \\
\hline $\mathrm{Fe} 2^{\mathrm{i}}-\mathrm{A} 15-\mathrm{Al1} 5^{\mathrm{i}}$ & $57.25(4)$ \\
\hline $\mathrm{Fe} 5-\mathrm{Al} 5-\mathrm{Al}^{\mathrm{vi}}$ & $121.06(4)$ \\
\hline $\mathrm{Fe}^{\mathrm{i}}-\mathrm{A} 15-\mathrm{Al}^{\mathrm{vi}}$ & $65.15(4)$ \\
\hline $\mathrm{A} 115^{\mathrm{i}}-\mathrm{A} 15-\mathrm{Al} 3^{\mathrm{vi}}$ & $58.41(3)$ \\
\hline $\mathrm{Fe} 5-\mathrm{Al} 5-\mathrm{Al}^{\mathrm{xxv}}$ & $121.06(4)$ \\
\hline $\mathrm{Fe} 2^{\mathrm{i}}-\mathrm{Al} 15-\mathrm{A} 13^{\mathrm{xxv}}$ & $65.15(4)$ \\
\hline $\mathrm{A} 115^{\mathrm{i}}-\mathrm{A} 15-\mathrm{A} 13^{\mathrm{xxv}}$ & $58.41(3)$ \\
\hline $\mathrm{A} 13^{\mathrm{vi}}-\mathrm{A} 15-\mathrm{A} 13^{\mathrm{xxv}}$ & $113.47(7)$ \\
\hline $\mathrm{Fe} 5-\mathrm{Al} 5-\mathrm{Al} 4^{\mathrm{vi}}$ & $61.19(5)$ \\
\hline $\mathrm{Fe} 2^{\mathrm{i}}-\mathrm{A} 15-\mathrm{A} 14^{\mathrm{vi}}$ & $138.96(7)$ \\
\hline $\mathrm{A} 115^{\mathrm{i}}-\mathrm{A} 15-\mathrm{A} 14^{\mathrm{vi}}$ & $81.70(6)$ \\
\hline $\mathrm{Al} 3^{\mathrm{vi}}-\mathrm{Al} 5-\mathrm{Al} 4^{\mathrm{vi}}$ & $94.91(5)$ \\
\hline $\mathrm{A} 13^{\mathrm{xxv}}-\mathrm{A} 15-\mathrm{A} 14^{\mathrm{vi}}$ & $94.91(5)$ \\
\hline $\mathrm{Fe} 5-\mathrm{A} 15-\mathrm{A} 114^{\mathrm{x}}$ & $60.26(3)$ \\
\hline $\mathrm{Fe} 2^{\mathrm{i}}-\mathrm{A} 15-\mathrm{A} 114^{\mathrm{x}}$ & $126.18(3)$ \\
\hline $\mathrm{A} 115^{\mathrm{i}}-\mathrm{A} 15-\mathrm{A} 114^{\mathrm{x}}$ & $100.22(5)$ \\
\hline $\mathrm{A} 13^{\mathrm{vi}}-\mathrm{A} 15-\mathrm{A} 114^{\mathrm{x}}$ & $148.06(7)$ \\
\hline $\mathrm{Al} 3^{\mathrm{xxy}}-\mathrm{A} 15-\mathrm{A} 114^{\mathrm{x}}$ & $61.87(3)$ \\
\hline $\mathrm{A} 14^{\mathrm{vi}}-\mathrm{A} 15-\mathrm{A} 114^{\mathrm{x}}$ & $56.22(3)$ \\
\hline $\mathrm{Fe} 5-\mathrm{Al} 5-\mathrm{Al} 14^{\mathrm{iv}}$ & $60.26(3)$ \\
\hline $\mathrm{Fe} 2^{\mathrm{i}}-\mathrm{A} 15-\mathrm{A} 114^{\mathrm{iv}}$ & $126.18(3)$ \\
\hline $\mathrm{A} 115^{\mathrm{i}}-\mathrm{A} 15-\mathrm{A} 114^{\mathrm{iv}}$ & $100.22(5)$ \\
\hline $\mathrm{Al} 3^{\mathrm{vi}}-\mathrm{A} 15-\mathrm{A} 114^{\mathrm{iv}}$ & $61.87(3)$ \\
\hline $\mathrm{A} 13^{\mathrm{xxv}}-\mathrm{A} 15-\mathrm{A} 114^{\mathrm{iv}}$ & $148.06(7)$ \\
\hline $\mathrm{Al} 4^{\mathrm{vi}}-\mathrm{Al} 5-\mathrm{Al} 14^{\mathrm{iv}}$ & $56.22(3)$ \\
\hline $\mathrm{A} 114^{\mathrm{x}}-\mathrm{A} 15-\mathrm{A} 114^{\mathrm{iv}}$ & $104.25(6)$ \\
\hline $\mathrm{Fe} 5-\mathrm{Al} 5-\mathrm{Al} 3^{\mathrm{iii}}$ & $54.52(4)$ \\
\hline $\mathrm{Fe} 2^{\mathrm{i}}-\mathrm{A} 15-\mathrm{A} 13^{\mathrm{iii}}$ & $110.94(5)$ \\
\hline $\mathrm{Al} 15^{\mathrm{i}}-\mathrm{Al} 5-\mathrm{Al} 3^{\mathrm{iii}}$ & $142.30(3)$ \\
\hline $\mathrm{Al} 3^{\mathrm{vi}}-\mathrm{Al} 5-\mathrm{Al}^{3 \mathrm{iii}}$ & $155.12(6)$ \\
\hline $\mathrm{Al} 3^{\mathrm{xxv}}-\mathrm{A} 15-\mathrm{A} 13^{\mathrm{iii}}$ & $83.93(3)$ \\
\hline $\mathrm{A} 14^{\mathrm{vi}}-\mathrm{A} 15-\mathrm{A} 13^{\mathrm{iii}}$ & $101.38(5)$ \\
\hline $\mathrm{Al} 14^{\mathrm{x}}-\mathrm{A} 15-\mathrm{Al} 3^{\mathrm{iii}}$ & $55.31(3)$ \\
\hline $\mathrm{A} 114^{\mathrm{iv}}-\mathrm{A} 15-\mathrm{A} 13^{\mathrm{iii}}$ & $112.77(6)$ \\
\hline $\mathrm{Fe} 5-\mathrm{A} 15-\mathrm{Al} 3$ & $54.52(4)$ \\
\hline $\mathrm{Fe} 2 \mathrm{i}-\mathrm{A} 15-\mathrm{A} 13$ & $110.94(5)$ \\
\hline $\mathrm{A} 115^{\mathrm{i}}-\mathrm{A} 15-\mathrm{A} 13$ & $142.30(3)$ \\
\hline $\mathrm{Al} 3^{\mathrm{vi}}-\mathrm{A} 15-\mathrm{Al} 3$ & $83.93(3)$ \\
\hline $\mathrm{Al} 3^{\mathrm{xxy}}-\mathrm{A} 15-\mathrm{A} 13$ & $155.12(6)$ \\
\hline $\mathrm{Al} 4^{\mathrm{vi}}-\mathrm{Al} 5-\mathrm{Al} 3$ & $101.38(5)$ \\
\hline $\mathrm{A} 114^{\mathrm{x}}-\mathrm{A} 15-\mathrm{A} 13$ & $112.77(6)$ \\
\hline $\mathrm{Al} 14^{\mathrm{iv}}-\mathrm{A} 15-\mathrm{A} 13$ & $55.31(3)$ \\
\hline 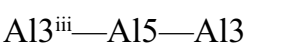 & $74.56(6)$ \\
\hline $\mathrm{Fe} 5-\mathrm{Al} 5-\mathrm{Al} 14^{\mathrm{ii}}$ & $113.06(5)$ \\
\hline $\mathrm{Fe} 2^{\mathrm{i}}-\mathrm{A} 15-\mathrm{A} 114^{\mathrm{ii}}$ & $52.54(3)$ \\
\hline
\end{tabular}

\begin{tabular}{|c|c|}
\hline $\mathrm{A} 18^{\mathrm{xiv}}-\mathrm{A} 113-\mathrm{A} 114$ & $98.32(4)$ \\
\hline Al12-Al13-Al14 & $60.45(4)$ \\
\hline $\mathrm{Fe} 3-\mathrm{A} 113-\mathrm{A} 110^{\mathrm{xiv}}$ & $126.27(5)$ \\
\hline $\mathrm{Fe} 4^{\mathrm{ii}}-\mathrm{A} 113-\mathrm{A} 110^{\mathrm{xiv}}$ & $75.96(4)$ \\
\hline $\mathrm{A} 14^{\mathrm{ix}}-\mathrm{A} 113-\mathrm{A} 110^{\mathrm{xiv}}$ & $61.51(4)$ \\
\hline $\mathrm{A} 19^{\mathrm{xiv}}-\mathrm{A} 113-\mathrm{A} 110^{\mathrm{xiv}}$ & $61.79(4)$ \\
\hline $\mathrm{Fe}^{\mathrm{xiv}}-\mathrm{A} 113-\mathrm{A} 110^{\mathrm{xiv}}$ & $51.60(3)$ \\
\hline $\mathrm{A} 18^{\mathrm{xiv}}-\mathrm{A} 113-\mathrm{A} 110^{\mathrm{xiv}}$ & $102.58(5)$ \\
\hline $\mathrm{A} 112-\mathrm{Al} 13-\mathrm{A} 110^{\mathrm{xiv}}$ & $162.64(5)$ \\
\hline $\mathrm{Al14}-\mathrm{Al} 13-\mathrm{Al} 10^{\mathrm{xiv}}$ & $116.75(5)$ \\
\hline $\mathrm{Fe} 3-\mathrm{Al13}-\mathrm{Al} 9$ & $112.75(4)$ \\
\hline $\mathrm{Fe} 44^{\mathrm{ii}}-\mathrm{Al13}-\mathrm{Al} 9$ & $55.60(3)$ \\
\hline $\mathrm{A} 14^{\mathrm{ix}}-\mathrm{A} 113-\mathrm{A} 19$ & $97.03(5)$ \\
\hline $\mathrm{A} 19^{\mathrm{xiv}}-\mathrm{A} 113-\mathrm{A} 19$ & $89.53(4)$ \\
\hline $\mathrm{Fe}^{\mathrm{xiv}}-\mathrm{A} 113-\mathrm{A} 19$ & $111.71(4)$ \\
\hline $\mathrm{A} 18^{\mathrm{xiv}}-\mathrm{A} 113-\mathrm{A} 19$ & $151.00(4)$ \\
\hline $\mathrm{Al12}-\mathrm{A} 113-\mathrm{Al} 9$ & $136.79(5)$ \\
\hline Al14-Al13-Al9 & $110.25(4)$ \\
\hline $\mathrm{A} 110^{\mathrm{xiv}}-\mathrm{A} 113-\mathrm{A} 19$ & $60.50(4)$ \\
\hline $\mathrm{Fe} 3-\mathrm{A} 113-\mathrm{A} 110$ & $53.55(3)$ \\
\hline $\mathrm{Fe} 4{ }^{\mathrm{ii}}-\mathrm{A} 113-\mathrm{Al} 10$ & $110.34(5)$ \\
\hline $\mathrm{A} 14^{\mathrm{ix}}-\mathrm{A} 113-\mathrm{A} 110$ & $152.92(5)$ \\
\hline $\mathrm{A} 19^{\mathrm{xiv}}-\mathrm{A} 113-\mathrm{A} 110$ & $61.43(4)$ \\
\hline $\mathrm{Fe} 1^{\mathrm{xiv}}-\mathrm{A} 113-\mathrm{A} 110$ & $115.34(4)$ \\
\hline $\mathrm{A} 18^{\mathrm{xiv}}-\mathrm{A} 113-\mathrm{A} 110$ & $101.55(5)$ \\
\hline Al12-Al13-Al10 & $98.54(4)$ \\
\hline Al14-Al13-Al10 & $139.16(5)$ \\
\hline $\mathrm{A} 110^{\mathrm{xiv}}-\mathrm{A} 113-\mathrm{A} 110$ & $93.19(3)$ \\
\hline Al9-Al13-Al10 & $59.51(4)$ \\
\hline $\mathrm{Fe} 2-\mathrm{Al} 14-\mathrm{Fe} 4^{\mathrm{ii}}$ & $146.58(5)$ \\
\hline $\mathrm{Fe} 2-\mathrm{Al} 14-\mathrm{Al} 3^{\mathrm{xxiii}}$ & $67.35(4)$ \\
\hline $\mathrm{Fe} 4^{\mathrm{ii}}-\mathrm{A} 114-\mathrm{A} 13^{\mathrm{xxiii}}$ & $130.88(5)$ \\
\hline $\mathrm{Fe} 2-\mathrm{A} 114-\mathrm{A} 14^{\mathrm{ix}}$ & $151.93(6)$ \\
\hline $\mathrm{Fe} 4^{\mathrm{ii}}-\mathrm{A} 114-\mathrm{A} 14^{\mathrm{ix}}$ & $56.56(4)$ \\
\hline $\mathrm{Al} 3^{\mathrm{xxiii}}-\mathrm{A} 114-\mathrm{Al} 4^{\mathrm{ix}}$ & $111.43(5)$ \\
\hline $\mathrm{Fe} 2-\mathrm{Al} 14-\mathrm{Fe}^{\mathrm{xxi}}$ & $103.42(4)$ \\
\hline $\mathrm{Fe} 4^{\mathrm{ii}}-\mathrm{Al} 14-\mathrm{Fe}^{\mathrm{xxi}}$ & $109.77(4)$ \\
\hline $\mathrm{Al} 3^{\mathrm{xxiii}}-\mathrm{A} 114-\mathrm{Fe} 5^{\mathrm{xxi}}$ & $54.95(3)$ \\
\hline $\mathrm{A} 14^{\mathrm{ix}}-\mathrm{A} 114-\mathrm{Fe} 5^{\mathrm{xxi}}$ & $60.50(4)$ \\
\hline $\mathrm{Cu} 2-\mathrm{Al} 14-\mathrm{Al} 13$ & $115.49(4)$ \\
\hline $\mathrm{Fe} 2-\mathrm{Al14}-\mathrm{A} 113$ & $115.49(4)$ \\
\hline $\mathrm{Fe} 4^{\mathrm{ii}}-\mathrm{Al14}-\mathrm{Al13}$ & $55.66(3)$ \\
\hline $\mathrm{Al} 3^{\mathrm{xxiii}}-\mathrm{A} 114-\mathrm{Al} 13$ & $164.61(5)$ \\
\hline $\mathrm{A} 14^{\mathrm{ix}}-\mathrm{A} 114-\mathrm{A} 113$ & $58.35(4)$ \\
\hline $\mathrm{Fe}^{\mathrm{xxi}}-\mathrm{Al14}-\mathrm{Al13}$ & $110.71(4)$ \\
\hline $\mathrm{Cu} 2-\mathrm{Al14}-\mathrm{Al12}$ & $56.18(4)$ \\
\hline $\mathrm{Fe} 2-\mathrm{Al} 14-\mathrm{A} 112$ & $56.18(4)$ \\
\hline $\mathrm{Fe} 4 \mathrm{ii}-\mathrm{A} 114-\mathrm{Al} 12$ & $109.50(5)$ \\
\hline
\end{tabular}




\begin{tabular}{|c|c|}
\hline $\mathrm{A} 115^{\mathrm{i}}-\mathrm{A} 15-\mathrm{A} 114^{\mathrm{ii}}$ & $96.26(5)$ \\
\hline $\mathrm{Al}^{\mathrm{vi}}-\mathrm{A} 15-\mathrm{A} 114^{\mathrm{ii}}$ & $55.04(3)$ \\
\hline $\mathrm{Al}^{\mathrm{xxv}}-\mathrm{Al} 5-\mathrm{A} 114^{\mathrm{ii}}$ & $115.39(6)$ \\
\hline 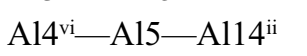 & $143.20(3)$ \\
\hline $\mathrm{All1} 4^{\mathrm{x}}-\mathrm{A} 15-\mathrm{Al} 14^{\mathrm{ii}}$ & $156.89(6)$ \\
\hline $\mathrm{A} 114^{\mathrm{iv}}-\mathrm{A} 15-\mathrm{A} 114^{\mathrm{ii}}$ & $88.51(3)$ \\
\hline 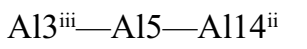 & $102.12(5)$ \\
\hline $\mathrm{Al} 3-\mathrm{Al} 5-\mathrm{Al1} 4^{\mathrm{ii}}$ & $58.95(4)$ \\
\hline $\mathrm{Fe} 5-\mathrm{A} 15-\mathrm{A} 114^{\mathrm{i}}$ & $113.06(5)$ \\
\hline $\mathrm{Fe} 2^{\mathrm{i}}-\mathrm{A} 15-\mathrm{A} 114^{\mathrm{i}}$ & $52.54(3)$ \\
\hline $\mathrm{Al} 15^{\mathrm{i}}-\mathrm{Al} 5-\mathrm{Al} 14^{\mathrm{i}}$ & $96.26(5)$ \\
\hline $\mathrm{Al}^{\mathrm{vi}}-\mathrm{Al} 5-\mathrm{Al} 14^{\mathrm{i}}$ & $115.39(6)$ \\
\hline $\mathrm{Al} 3^{\mathrm{xxv}}-\mathrm{A} 15-\mathrm{A} 114^{\mathrm{i}}$ & $55.04(3)$ \\
\hline $\mathrm{A} 14^{\mathrm{vi}}-\mathrm{A} 15-\mathrm{A} 114^{\mathrm{i}}$ & $143.20(3)$ \\
\hline $\mathrm{Al} 14^{\mathrm{x}}-\mathrm{Al} 5-\mathrm{A} 114^{\mathrm{i}}$ & $88.51(3)$ \\
\hline $\mathrm{A} 114^{\mathrm{iv}}-\mathrm{A} 15-\mathrm{A} 114^{\mathrm{i}}$ & $156.89(6)$ \\
\hline 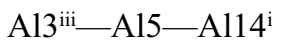 & $58.95(4)$ \\
\hline $\mathrm{Al} 3-\mathrm{Al} 5-\mathrm{A} 114^{\mathrm{i}}$ & $102.12(5)$ \\
\hline $\mathrm{Al1} 4^{\mathrm{ii}}-\mathrm{A} 15-\mathrm{A} 114^{\mathrm{i}}$ & $73.60(5)$ \\
\hline $\mathrm{Fe} 5-\mathrm{A} 11-\mathrm{Fe}^{\mathrm{i}}$ & 180.0 \\
\hline $\mathrm{Fe} 5-\mathrm{Al1}-\mathrm{Al} 3$ & $56.71(2)$ \\
\hline $\mathrm{Fe} 5^{\mathrm{i}}-\mathrm{A} 11-\mathrm{A} 13$ & $123.29(2)$ \\
\hline $\mathrm{Fe} 5-\mathrm{A} 11-\mathrm{Al}^{\mathrm{i}}$ & $123.29(2)$ \\
\hline $\mathrm{Fe} 5^{\mathrm{i}}-\mathrm{Al} 1-\mathrm{Al} 3^{\mathrm{i}}$ & $56.71(2)$ \\
\hline $\mathrm{Al} 3-\mathrm{Al} 1-\mathrm{Al}^{\mathrm{i}}$ & 180.0 \\
\hline $\mathrm{Fe} 5-\mathrm{A} 11-\mathrm{Al} 3^{\mathrm{iii}}$ & $56.71(2)$ \\
\hline $\mathrm{Fe} 5^{\mathrm{i}}-\mathrm{A} 11-\mathrm{A} 13^{\mathrm{iii}}$ & $123.29(2)$ \\
\hline $\mathrm{A} 13-\mathrm{A} 11-\mathrm{Al} 3^{\mathrm{iii}}$ & $80.93(5)$ \\
\hline $\mathrm{Al} 3^{\mathrm{i}}-\mathrm{A} 11-\mathrm{Al} 3^{\mathrm{iii}}$ & $99.07(5)$ \\
\hline $\mathrm{Fe} 5-\mathrm{Al} 1-\mathrm{Al} 3^{\mathrm{ii}}$ & $123.29(2)$ \\
\hline $\mathrm{Fe} 5^{\mathrm{i}}-\mathrm{A} 11-\mathrm{Al} 3^{\mathrm{ii}}$ & $56.71(2)$ \\
\hline $\mathrm{A} 13-\mathrm{A} 11-\mathrm{A} 13^{\mathrm{ii}}$ & $99.07(5)$ \\
\hline $\mathrm{Al} 3^{\mathrm{i}}-\mathrm{A} 11-\mathrm{Al} 3^{\mathrm{ii}}$ & $80.93(5)$ \\
\hline $\mathrm{A} 13^{\mathrm{iii}}-\mathrm{A} 11-\mathrm{A} 13^{\mathrm{ii}}$ & $180.00(4)$ \\
\hline $\mathrm{Fe} 5-\mathrm{Al} 1-\mathrm{Al} 6$ & $54.96(2)$ \\
\hline $\mathrm{Fe} 5^{\mathrm{i}}-\mathrm{A} 11-\mathrm{Al} 6$ & $125.04(2)$ \\
\hline $\mathrm{Al3}-\mathrm{Al1}-\mathrm{Al} 6$ & $111.58(3)$ \\
\hline $\mathrm{A} 13^{\mathrm{i}}-\mathrm{A} 11-\mathrm{Al} 6$ & $68.42(3)$ \\
\hline $\mathrm{A} 13^{\mathrm{iii}}-\mathrm{A} 11-\mathrm{Al} 6$ & $60.17(3)$ \\
\hline $\mathrm{A} 13^{\mathrm{ii}}-\mathrm{A} 11-\mathrm{Al} 6$ & $119.83(3)$ \\
\hline $\mathrm{Fe} 5-\mathrm{A} 11-\mathrm{A} 16^{\mathrm{i}}$ & $125.04(2)$ \\
\hline $\mathrm{Fe} 5^{\mathrm{i}}-\mathrm{A} 11-\mathrm{Al}^{\mathrm{i}}$ & $54.96(2)$ \\
\hline $\mathrm{A} 13-\mathrm{A} 11-\mathrm{Al}^{\mathrm{i}}$ & $68.42(3)$ \\
\hline $\mathrm{A} 13^{\mathrm{i}}-\mathrm{A} 11-\mathrm{A} 16^{\mathrm{i}}$ & $111.58(3)$ \\
\hline $\mathrm{A} 13^{\mathrm{iii}}-\mathrm{A} 11-\mathrm{A} 16^{\mathrm{i}}$ & $119.83(3)$ \\
\hline $\mathrm{A} 13^{\mathrm{ii}}-\mathrm{A} 11-\mathrm{Al}^{\mathrm{i}}$ & $60.17(3)$ \\
\hline $\mathrm{A} 16-\mathrm{A} 11-\mathrm{Al} 6^{\mathrm{i}}$ & 180.0 \\
\hline $\mathrm{Fe} 5-\mathrm{A} 11-\mathrm{Al} 6^{\mathrm{ii}}$ & 125.04 \\
\hline
\end{tabular}

Al3 ${ }^{\text {xxiii-Al14-Al12 } 118.96(5)}$

Al4 ${ }^{\mathrm{ix}}$-Al14-Al12

$\mathrm{Fe} 5^{\text {xxi-Al14-Al12 }}$

Al13-Al14-Al12

$\mathrm{Fe} 2-\mathrm{A} 114-\mathrm{Al}^{\text {xxi }}$

$\mathrm{Fe} 4$ ii-Al14-Al5 ${ }^{\mathrm{xxi}}$

Al3 ${ }^{\text {xiii }}-\mathrm{A} 114-\mathrm{A} 15^{\mathrm{xxi}}$

$\mathrm{A} 14^{\mathrm{ix}}-\mathrm{A} 114-\mathrm{A} 15^{\mathrm{xxi}}$

$\mathrm{Fe} 5^{\text {xxi }}-\mathrm{A} 114-\mathrm{A} 15^{\mathrm{xxi}}$

Al13-A114-A15 ${ }^{\text {xi }}$

Al12-Al14-Al5 ${ }^{\text {xi }}$

$\mathrm{Fe} 2-\mathrm{A} 114-\mathrm{Al}^{3 i \mathrm{i}}$

$\mathrm{Fe} 4^{\mathrm{ii}}-\mathrm{Al14}-\mathrm{Al} 3^{\mathrm{ii}}$

$\mathrm{Al} 3^{\mathrm{xxii}}-\mathrm{A} 114-\mathrm{Al}^{3 \mathrm{ii}}$

$\mathrm{A} 14^{\mathrm{ix}}-\mathrm{A} 114-\mathrm{A} 13^{\mathrm{ii}}$

$\mathrm{Fe}^{5 \times \mathrm{xi}}-\mathrm{A} 114-\mathrm{Al}^{\mathrm{ii}}$

Al13-Al14-Al3 ${ }^{\mathrm{ii}}$

Al12-Al14-Al3 ${ }^{\mathrm{ii}}$

$\mathrm{Al5}{ }^{\mathrm{xxi}}-\mathrm{A} 114-\mathrm{Al}^{3 \mathrm{ii}}$

$\mathrm{Fe} 2-\mathrm{A} 114-\mathrm{Al6}^{\mathrm{xxi}}$

$\mathrm{Fe} 4^{\mathrm{ii}}-\mathrm{A} 114-\mathrm{Al}^{\mathrm{xxi}}$

Al3 ${ }^{\text {xxiii-A }}$ Al14-Al6 ${ }^{\text {xxi }}$

$\mathrm{A} 14^{\mathrm{ix}}-\mathrm{A} 114-\mathrm{Al} 6^{\mathrm{xxi}}$

$\mathrm{Fe} 5^{\text {xxi }}$-Al14-Al6 ${ }^{\text {xxi }}$

Al13-Al14-Al6 ${ }^{\text {xxi }}$

Al12-Al14-Al6 ${ }^{\text {xxi }}$

A15 ${ }^{\text {xxi }}$-A114-A16 ${ }^{\text {xxi }}$

$\mathrm{Al} 3^{\mathrm{ii}}-\mathrm{A} 114-\mathrm{Al}^{\mathrm{xxi}}$

$\mathrm{Fe} 2-\mathrm{Al} 14-\mathrm{Al} 5^{\mathrm{i}}$

$\mathrm{Fe} 4^{\mathrm{ii}}$-Al14-Al5

$\mathrm{Al} 3^{\text {xxii }}-\mathrm{Al} 14-\mathrm{Al} 5^{\mathrm{i}}$

$\mathrm{A} 14^{\mathrm{ix}}-\mathrm{A} 114-\mathrm{A} 15^{\mathrm{i}}$

$\mathrm{Fe}^{\mathrm{xxi}}-\mathrm{A} 114-\mathrm{A} 15^{\mathrm{i}}$

Al13-Al14-A15 ${ }^{\mathrm{i}}$

$\mathrm{A} 112-\mathrm{A} 114-\mathrm{Al} 5^{\mathrm{i}}$

$\mathrm{A} 15^{\mathrm{xxi}}-\mathrm{A} 114-\mathrm{Al}^{\mathrm{i}}$

$\mathrm{Al} 3^{\mathrm{ii}}$-A $114-\mathrm{A} 15^{\mathrm{i}}$

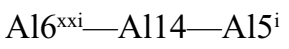

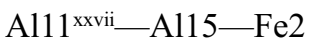

Al11 ${ }^{\text {xxvi }-A 115-C u 2 ~}$

Al11 $1^{\text {xxvi }-A 115-A 115 ~}{ }^{\text {xxii }}$

$108.01(5)$

$116.70(5)$

$59.76(4)$

$129.43(5)$

$72.10(4)$

$62.69(5)$

$61.30(4)$

$52.17(3)$

$114.73(5)$

$166.75(5)$

112.32 (4)

$53.16(3)$

$85.41(4)$

$95.21(5)$

108.64 (4)

106.17 (4)

$134.62(5)$

$57.31(4)$

57.05 (3)

$153.47(5)$

$58.87(4)$

$97.32(5)$

$51.84(3)$

$108.91(4)$

$71.69(4)$

$100.84(5)$

144.27 (5)

54.29 (3)

$107.32(5)$

$58.03(4)$

150.77 (5)

112.58 (4)

$136.71(5)$

100.31 (4)

91.49 (3)

58.60 (4)

98.27 (4)

$172.31(7)$

$172.31(7)$

125.05 (9)

62.65 (6)

58.39 (4)

116.38 (4)

139.47 (3)

58.39 (4)

116.38 (4)

139.47 (3) 


\begin{tabular}{|c|c|}
\hline $\mathrm{Fe} 5^{\mathrm{i}}-\mathrm{Al} 1-\mathrm{A} 16^{\mathrm{ii}}$ & $54.96(2)$ \\
\hline $\mathrm{A} 13-\mathrm{A} 11-\mathrm{A} 16^{\mathrm{ii}}$ & $119.83(3)$ \\
\hline $\mathrm{A} 13^{\mathrm{i}}-\mathrm{A} 11-\mathrm{A} 16^{\mathrm{ii}}$ & $60.17(3)$ \\
\hline 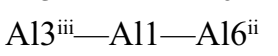 & $68.42(3)$ \\
\hline $\mathrm{Al} 3^{\mathrm{ii}}-\mathrm{A} 11-\mathrm{Al} 6^{\mathrm{ii}}$ & $111.58(3)$ \\
\hline $\mathrm{A} 16-\mathrm{A} 11-\mathrm{Al} 6^{\mathrm{ii}}$ & $96.39(4)$ \\
\hline $\mathrm{A} 16^{\mathrm{i}}-\mathrm{A} 11-\mathrm{A} 16^{\mathrm{ii}}$ & $83.62(4)$ \\
\hline $\mathrm{Fe} 5-\mathrm{All}-\mathrm{Al} 6^{\mathrm{iii}}$ & $54.96(2)$ \\
\hline $\mathrm{Fe} 5^{\mathrm{i}}-\mathrm{A} 11-\mathrm{A} 16^{\mathrm{iii}}$ & $125.04(2)$ \\
\hline $\mathrm{A} 13-\mathrm{A} 11-\mathrm{A} 16^{\mathrm{iii}}$ & $60.17(3)$ \\
\hline $\mathrm{A} 13^{\mathrm{i}}-\mathrm{A} 11-\mathrm{A} 16^{\mathrm{iii}}$ & $119.83(3)$ \\
\hline $\mathrm{A} 13^{\mathrm{iii}-\mathrm{A}} 11-\mathrm{A} 16^{\mathrm{iii}}$ & $111.58(3)$ \\
\hline $\mathrm{A} 13^{\mathrm{ii}}-\mathrm{A} 11-\mathrm{A} 16^{\mathrm{iii}}$ & $68.42(3)$ \\
\hline $\mathrm{A} 16-\mathrm{A} 11-\mathrm{Al} 6^{\mathrm{iii}}$ & $83.61(4)$ \\
\hline $\mathrm{A} 16^{\mathrm{i}}-\mathrm{A} 11-\mathrm{A} 16^{\mathrm{iii}}$ & $96.38(4)$ \\
\hline $\mathrm{A} 16^{\mathrm{ii}}-\mathrm{A} 11-\mathrm{A} 16^{\mathrm{iii}}$ & 180.0 \\
\hline $\mathrm{Fe} 5-\mathrm{Cu} 1-\mathrm{Fe} 5^{\mathrm{i}}$ & 180.0 \\
\hline $\mathrm{Fe} 5-\mathrm{Cu} 1-\mathrm{Al} 3$ & $56.71(2)$ \\
\hline $\mathrm{Fe} 5$ - $-\mathrm{Cu} 1-\mathrm{A} 13$ & $123.29(2)$ \\
\hline $\mathrm{Fe} 5-\mathrm{Cu} 1-\mathrm{Al}^{\mathrm{i}}$ & $123.29(2)$ \\
\hline $\mathrm{Fe} 5^{\mathrm{i}}-\mathrm{Cu} 1-\mathrm{Al} 3^{\mathrm{i}}$ & $56.71(2)$ \\
\hline $\mathrm{A} 13-\mathrm{Cu} 1-\mathrm{Al} 3^{\mathrm{i}}$ & 180.0 \\
\hline $\mathrm{Fe} 5-\mathrm{Cu} 1-\mathrm{Al}^{3}{ }^{\mathrm{iii}}$ & $56.71(2)$ \\
\hline 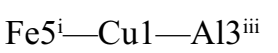 & $123.29(2)$ \\
\hline $\mathrm{Al} 3-\mathrm{Cu} 1-\mathrm{A} 13^{\mathrm{iii}}$ & $80.93(5)$ \\
\hline $\mathrm{Al} 3^{\mathrm{i}}-\mathrm{Cu} 1-\mathrm{A} 13^{\mathrm{iii}}$ & $99.07(5)$ \\
\hline $\mathrm{Fe} 5-\mathrm{Cu} 1-\mathrm{Al}^{3 \mathrm{ii}}$ & $123.29(2)$ \\
\hline $\mathrm{Fe} 5^{\mathrm{i}}-\mathrm{Cu} 1-\mathrm{A} 13^{\mathrm{ii}}$ & $56.71(2)$ \\
\hline $\mathrm{Al} 3-\mathrm{Cu} 1-\mathrm{Al}^{\mathrm{ii}}$ & $99.07(5)$ \\
\hline $\mathrm{Al} 3^{\mathrm{i}}-\mathrm{Cu} 1-\mathrm{A} 13^{\mathrm{ii}}$ & $80.93(5)$ \\
\hline $\mathrm{Al} 3^{\mathrm{iii}}-\mathrm{Cu} 1-\mathrm{A} 13^{\mathrm{ii}}$ & $180.00(4)$ \\
\hline $\mathrm{Fe} 5-\mathrm{Cu} 1-\mathrm{Al} 6$ & $54.96(2)$ \\
\hline $\mathrm{Fe} 5-\mathrm{Cu} 1-\mathrm{Al} 6$ & $125.04(2)$ \\
\hline $\mathrm{Al} 3-\mathrm{Cu} 1-\mathrm{Al} 6$ & $111.58(3)$ \\
\hline $\mathrm{Al} 3^{\mathrm{i}}-\mathrm{Cu} 1-\mathrm{Al} 6$ & $68.42(3)$ \\
\hline 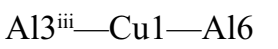 & $60.17(3)$ \\
\hline $\mathrm{Al} 3^{\mathrm{ii}}-\mathrm{Cu} 1-\mathrm{A} 16$ & $119.83(3)$ \\
\hline $\mathrm{Fe} 5-\mathrm{Cu} 1-\mathrm{A} 16^{\mathrm{i}}$ & $125.04(2)$ \\
\hline $\mathrm{Fe} 5^{\mathrm{i}}-\mathrm{Cu} 1-\mathrm{Al}^{\mathrm{i}}$ & $54.96(2)$ \\
\hline $\mathrm{Al} 3-\mathrm{Cu} 1-\mathrm{Al} 6^{\mathrm{i}}$ & $68.42(3)$ \\
\hline $\mathrm{Al} 3^{\mathrm{i}}-\mathrm{Cu} 1-\mathrm{A} 16^{\mathrm{i}}$ & $111.58(3)$ \\
\hline $\mathrm{Al} 3^{\mathrm{iii}}-\mathrm{Cu} 1-\mathrm{A} 16^{\mathrm{i}}$ & $119.83(3)$ \\
\hline $\mathrm{A} 13^{\mathrm{ii}}-\mathrm{Cu} 1-\mathrm{A} 16^{\mathrm{i}}$ & $60.17(3)$ \\
\hline $\mathrm{A} 16-\mathrm{Cu} 1-\mathrm{A} 16^{\mathrm{i}}$ & 180.0 \\
\hline $\mathrm{Fe} 5-\mathrm{Cu} 1-\mathrm{Al} 6^{\mathrm{ii}}$ & $125.04(2)$ \\
\hline $\mathrm{Fe} 5^{\mathrm{i}}-\mathrm{Cu} 1-\mathrm{A} 16^{\mathrm{ii}}$ & $54.96(2)$ \\
\hline $\mathrm{Al} 3-\mathrm{Cu} 1-\mathrm{Al} 6^{\mathrm{ii}}$ & $119.83(3)$ \\
\hline $\mathrm{Al} 3^{\mathrm{i}}-\mathrm{Cu} 1-\mathrm{A} 16^{\mathrm{ii}}$ & $60.17(3)$ \\
\hline
\end{tabular}

\begin{tabular}{|c|c|}
\hline $\mathrm{Fe} 4^{\mathrm{xxiii}}-\mathrm{A} 115-\mathrm{Fe} 4^{\mathrm{xxiv}}$ & $79.73(4)$ \\
\hline $\mathrm{A} 111^{\mathrm{xxvii}}-\mathrm{A} 115-\mathrm{Fe} 2^{\mathrm{xxii}}$ & $66.65(5)$ \\
\hline $\mathrm{Fe} 2-\mathrm{A} 115-\mathrm{Fe} 2^{\mathrm{xxii}}$ & $121.04(5)$ \\
\hline $\mathrm{A} 115^{\mathrm{xxi}}-\mathrm{A} 115-\mathrm{Fe} 2^{\mathrm{xxii}}$ & $58.40(5)$ \\
\hline $\mathrm{Fe} 4^{\mathrm{xxii}}-\mathrm{A} 115-\mathrm{Fe}^{2 \mathrm{xii}}$ & $107.89(4)$ \\
\hline $\mathrm{Fe} 4^{\mathrm{xxiv}}-\mathrm{A} 115-\mathrm{Fe} 2^{\mathrm{xxii}}$ & $107.89(4)$ \\
\hline $\mathrm{Al11}{ }^{\mathrm{xxvii}}-\mathrm{A} 115-\mathrm{Al} 3^{\mathrm{xxiii}}$ & $110.88(4)$ \\
\hline $\mathrm{Fe} 2-\mathrm{A} 115-\mathrm{Al} 3^{\mathrm{xxiii}}$ & $66.27(3)$ \\
\hline $\mathrm{Al} 15^{\mathrm{xxii}}-\mathrm{A} 115-\mathrm{Al} 3^{\mathrm{xxiii}}$ & $95.53(5)$ \\
\hline $\mathrm{Fe} 4^{\mathrm{xxiii}}-\mathrm{Al} 15-\mathrm{Al} 3^{\mathrm{xxiii}}$ & $55.25(3)$ \\
\hline $\mathrm{Fe} 4^{\mathrm{xxiv}}-\mathrm{Al} 15-\mathrm{Al} 3^{\mathrm{xxiii}}$ & $122.08(6)$ \\
\hline $\mathrm{Fe} 2^{\mathrm{xxii}}-\mathrm{A} 115-\mathrm{Al} 3^{\mathrm{xxiii}}$ & $118.62(3)$ \\
\hline $\mathrm{A} 111^{\mathrm{xxvii}}-\mathrm{A} 115-\mathrm{A} 13^{\mathrm{xxiv}}$ & $110.88(4)$ \\
\hline $\mathrm{Fe} 2-\mathrm{A} 115-\mathrm{Al} 3^{\mathrm{xxiv}}$ & $66.26(3)$ \\
\hline $\mathrm{A} 115^{\mathrm{xxii}}-\mathrm{A} 115-\mathrm{Al} 3^{\mathrm{xxiv}}$ & $95.53(5)$ \\
\hline $\mathrm{Fe} 4^{\mathrm{xxiii}}-\mathrm{A} 115-\mathrm{A} 13^{\mathrm{xxiv}}$ & $122.08(6)$ \\
\hline $\mathrm{Fe} 4^{\text {xiiv }}-\mathrm{A} 115-\mathrm{Al} 3^{\text {xiv }}$ & $55.25(3)$ \\
\hline $\mathrm{Fe} 2^{\mathrm{xxii}}-\mathrm{A} 115-\mathrm{Al} 3^{\mathrm{xxiv}}$ & $118.62(3)$ \\
\hline $\mathrm{A} 13^{\mathrm{xxiii}}-\mathrm{A} 115-\mathrm{A} 13^{\mathrm{xxiv}}$ & $118.38(7)$ \\
\hline $\mathrm{A} 111^{\mathrm{xxvii}}-\mathrm{A} 115-\mathrm{A} 15^{\mathrm{i}}$ & $115.05(7)$ \\
\hline $\mathrm{Fe} 2-\mathrm{Al} 15-\mathrm{Al} 5^{\mathrm{i}}$ & $57.26(4)$ \\
\hline Al15 ${ }^{\mathrm{xxii}}-\mathrm{A} 115-\mathrm{A} 15^{\mathrm{i}}$ & $119.91(8)$ \\
\hline $\mathrm{Fe} 4^{\mathrm{xxiii}}-\mathrm{A} 115-\mathrm{A} 15^{\mathrm{i}}$ & $73.37(4)$ \\
\hline $\mathrm{Fe} 4^{\mathrm{xiv}}-\mathrm{A} 115-\mathrm{A} 15^{\mathrm{i}}$ & $73.37(4)$ \\
\hline $\mathrm{Fe} 2^{\mathrm{xxii}}-\mathrm{A} 115-\mathrm{A} 15^{\mathrm{i}}$ & $178.30(7)$ \\
\hline $\mathrm{Al}^{\mathrm{xxiii}}-\mathrm{A} 115-\mathrm{A} 15^{\mathrm{i}}$ & $61.05(3)$ \\
\hline $\mathrm{Al}^{\mathrm{xxiv}}-\mathrm{A} 115-\mathrm{A} 15^{\mathrm{i}}$ & $61.05(3)$ \\
\hline Al11 ${ }^{\mathrm{xxvii}}-\mathrm{A} 115-\mathrm{A} 16^{\mathrm{xxviii}}$ & $60.97(4)$ \\
\hline $\mathrm{Fe} 2-\mathrm{Al} 15-\mathrm{Al} 6^{\mathrm{xxviii}}$ & $122.12(4)$ \\
\hline $\mathrm{Al15} 5^{\mathrm{xxii}}-\mathrm{A} 115-\mathrm{A} 16^{\mathrm{xxviii}}$ & $88.17(5)$ \\
\hline $\mathrm{Fe} 4^{\mathrm{xxiii}}-\mathrm{A} 115-\mathrm{Al} 6^{\mathrm{xxviii}}$ & $56.87(3)$ \\
\hline $\mathrm{Fe} 4^{\mathrm{xxi}}-\mathrm{A} 115-\mathrm{A} 16^{\mathrm{xxviii}}$ & $117.61(5)$ \\
\hline $\mathrm{Fe} 2^{\mathrm{xxii}}-\mathrm{A} 115-\mathrm{Al} 6^{\mathrm{xxviii}}$ & $57.27(3)$ \\
\hline $\mathrm{Al} 3^{\mathrm{xxiii}}-\mathrm{A} 115-\mathrm{A} 16^{\mathrm{xxviii}}$ & $68.65(3)$ \\
\hline $\mathrm{A} 13^{\mathrm{xxi}}-\mathrm{A} 115-\mathrm{A} 16^{\mathrm{xxviii}}$ & $171.54(6)$ \\
\hline $\mathrm{A} 15^{\mathrm{i}}-\mathrm{A} 115-\mathrm{A} 16^{\mathrm{xxviii}}$ & $123.31(4)$ \\
\hline Al11 $1_{x v i i}-A 115-A 16^{x i i}$ & $60.97(4)$ \\
\hline $\mathrm{Fe} 2-\mathrm{A} 115-\mathrm{Al} 6^{\mathrm{xii}}$ & $122.12(4)$ \\
\hline $\mathrm{A} 115^{\mathrm{xxii}}-\mathrm{A} 115-\mathrm{A} 16^{\mathrm{xii}}$ & $88.17(5)$ \\
\hline $\mathrm{Fe} 4^{\mathrm{xxiii}}-\mathrm{A} 115-\mathrm{A} 16^{\mathrm{xii}}$ & $117.61(5)$ \\
\hline $\mathrm{Fe} 4^{\mathrm{xxiv}}-\mathrm{A} 115-\mathrm{A} 16^{\mathrm{xii}}$ & $56.87(3)$ \\
\hline $\mathrm{Fe} 2^{\mathrm{xxii}}-\mathrm{A} 115-\mathrm{Al} 6^{\mathrm{xii}}$ & $57.27(3)$ \\
\hline $\mathrm{A} 13^{\mathrm{xxiii}}-\mathrm{A} 115-\mathrm{Al} 6^{\mathrm{xii}}$ & $171.54(6)$ \\
\hline $\mathrm{A} 13^{\mathrm{xiv}}-\mathrm{A} 115-\mathrm{A} 16^{\mathrm{xii}}$ & $68.65(3)$ \\
\hline $\mathrm{A} 15^{\mathrm{i}}-\mathrm{A} 115-\mathrm{A} 16^{\mathrm{xii}}$ & $123.31(4)$ \\
\hline $\mathrm{A} 16^{\mathrm{xxvii}}-\mathrm{A} 115-\mathrm{A} 16^{\mathrm{xii}}$ & $103.96(6)$ \\
\hline $\mathrm{A} 12-\mathrm{Fe} 4-\mathrm{A} 13$ & $74.52(5)$ \\
\hline $\mathrm{A} 12-\mathrm{Fe} 4-\mathrm{A} 14$ & $133.36(5)$ \\
\hline
\end{tabular}




\begin{tabular}{|c|c|}
\hline $\mathrm{Al} 3^{\mathrm{iii}}-\mathrm{Cu} 1-\mathrm{Al}^{\mathrm{ii}}$ & $68.42(3)$ \\
\hline $\mathrm{A} 13^{\mathrm{ii}}-\mathrm{Cu} 1-\mathrm{A} 16^{\mathrm{ii}}$ & $111.58(3)$ \\
\hline $\mathrm{A} 16-\mathrm{Cu} 1-\mathrm{Al} 6^{\mathrm{ii}}$ & $96.39(4)$ \\
\hline $\mathrm{A} 16^{\mathrm{i}}-\mathrm{Cu} 1-\mathrm{A} 16^{\mathrm{ii}}$ & $83.62(4)$ \\
\hline $\mathrm{Fe} 5-\mathrm{Cu} 1-\mathrm{Al} 6^{\mathrm{iii}}$ & $54.96(2)$ \\
\hline $\mathrm{Fe} 5^{\mathrm{i}}-\mathrm{Cu} 1-\mathrm{A} 16^{\mathrm{iii}}$ & $125.04(2)$ \\
\hline $\mathrm{Al} 3-\mathrm{Cu} 1-\mathrm{Al} 6^{\mathrm{iii}}$ & $60.17(3)$ \\
\hline $\mathrm{Al} 3^{\mathrm{i}}-\mathrm{Cu} 1-\mathrm{Al} 6^{\mathrm{iii}}$ & $119.83(3)$ \\
\hline $\mathrm{Al} 3^{\mathrm{iii}}-\mathrm{Cu} 1-\mathrm{Al} 6^{\mathrm{iii}}$ & $111.58(3)$ \\
\hline $\mathrm{Al} 3^{\mathrm{ii}}-\mathrm{Cu} 1-\mathrm{Al} 6^{\mathrm{iii}}$ & $68.42(3)$ \\
\hline $\mathrm{Al} 6-\mathrm{Cu} 1-\mathrm{Al} 6^{\mathrm{iii}}$ & $83.61(4)$ \\
\hline $\mathrm{A} 16^{\mathrm{i}}-\mathrm{Cu} 1-\mathrm{A} 16^{\mathrm{iii}}$ & $96.38(4)$ \\
\hline $\mathrm{A} 16^{\mathrm{ii}}-\mathrm{Cu} 1-\mathrm{A} 16^{\mathrm{iii}}$ & 180.0 \\
\hline $\mathrm{Fe} 1-\mathrm{Al} 7-\mathrm{Fe} 5$ & $162.97(7)$ \\
\hline $\mathrm{Fe} 1-\mathrm{Al} 7-\mathrm{Al} 4^{\mathrm{vi}}$ & $132.34(7)$ \\
\hline $\mathrm{Fe} 5-\mathrm{Al} 7-\mathrm{Al} 4^{\mathrm{vi}}$ & $64.70(5)$ \\
\hline $\mathrm{Fe} 1-\mathrm{A} 17-\mathrm{Fe}^{\mathrm{xiii}}$ & $71.01(4)$ \\
\hline $\mathrm{Fe} 5-\mathrm{Al} 7-\mathrm{Fe}^{\mathrm{xiii}}$ & $126.02(6)$ \\
\hline $\mathrm{Al} 4^{\mathrm{vi}}-\mathrm{Al} 7-\mathrm{Fe}^{\mathrm{xiii}}$ & $61.32(5)$ \\
\hline $\mathrm{Fe} 1-\mathrm{Al} 7-\mathrm{Al} 9$ & $55.27(3)$ \\
\hline $\mathrm{Fe} 5-\mathrm{Al} 7-\mathrm{A} 19$ & $112.48(5)$ \\
\hline $\mathrm{A} 14^{\mathrm{vi}}-\mathrm{A} 17-\mathrm{A} 19$ & $142.52(3)$ \\
\hline $\mathrm{Fe}^{\mathrm{xiii}}-\mathrm{A} 17-\mathrm{A} 19$ & $109.81(5)$ \\
\hline $\mathrm{Fe} 1-\mathrm{A} 17-\mathrm{A} 19^{\mathrm{iii}}$ & $55.27(3)$ \\
\hline Fe5-A17-A19 ${ }^{\mathrm{iii}}$ & $112.48(5)$ \\
\hline $\mathrm{Al} 4^{\mathrm{vi}}-\mathrm{A} 17-\mathrm{A} 19^{\mathrm{iii}}$ & $142.52(3)$ \\
\hline $\mathrm{Fe} 1^{\mathrm{xii}}-\mathrm{A} 17-\mathrm{A} 19^{\mathrm{iii}}$ & $109.81(5)$ \\
\hline $\mathrm{Al} 9-\mathrm{Al} 7-\mathrm{A} 19^{\mathrm{iii}}$ & $74.70(5)$ \\
\hline Fe1-Al7-Al6 & $114.77(5)$ \\
\hline Fe5-A17-A16 & $54.07(3)$ \\
\hline $\mathrm{A} 14^{\mathrm{vi}}-\mathrm{A} 17-\mathrm{A} 16$ & $100.82(5)$ \\
\hline $\mathrm{Fe}^{\mathrm{xiii}}$-A17-Al6 & $137.51(3)$ \\
\hline A19-A17-Al6 & $106.24(5)$ \\
\hline $\mathrm{A} 19^{\mathrm{iii}}-\mathrm{Al} 7-\mathrm{Al} 6$ & $59.53(3)$ \\
\hline $\mathrm{Fe} 1-\mathrm{Al} 7-\mathrm{Al} 6^{\mathrm{iii}}$ & $114.77(5)$ \\
\hline $\mathrm{Fe} 5-\mathrm{Al} 7-\mathrm{Al} 6^{\mathrm{iii}}$ & $54.07(3)$ \\
\hline $\mathrm{Al} 4^{\mathrm{vi}}-\mathrm{A} 17-\mathrm{Al}^{\mathrm{iii}}$ & $100.82(5)$ \\
\hline $\mathrm{Fe} 1^{\mathrm{xii}}-\mathrm{Al} 7-\mathrm{Al} 6^{\mathrm{iii}}$ & $137.51(3)$ \\
\hline $\mathrm{Al} 9-\mathrm{A} 17-\mathrm{Al}^{\mathrm{iii}}$ & $59.53(3)$ \\
\hline $\mathrm{Al} 9^{\mathrm{iii}}-\mathrm{Al} 7-\mathrm{Al} 6^{\mathrm{iii}}$ & $106.24(5)$ \\
\hline Al6-Al7-Al6iii & $80.85(6)$ \\
\hline $\mathrm{Fe} 1-\mathrm{A} 17-\mathrm{A} 18^{\mathrm{xiii}}$ & $54.92(3)$ \\
\hline $\mathrm{Fe} 5-\mathrm{A} 17-\mathrm{Al} 8^{\mathrm{xiii}}$ & $132.33(3)$ \\
\hline $\mathrm{Al} 4^{\mathrm{vi}}-\mathrm{A} 17-\mathrm{Al} 8^{\mathrm{xiii}}$ & $94.27(4)$ \\
\hline $\mathrm{Fe} 1^{\mathrm{xii}}-\mathrm{A} 17-\mathrm{A} 18^{\mathrm{xiii}}$ & $54.06(3)$ \\
\hline $\mathrm{A} 19-\mathrm{A} 17-\mathrm{A} 18^{\mathrm{xiii}}$ & $109.39(5)$ \\
\hline $\mathrm{A} 19^{\mathrm{iii}}-\mathrm{A} 17-\mathrm{A} 18^{\mathrm{xiii}}$ & $58.81(3)$ \\
\hline $\mathrm{A} 16-\mathrm{A} 17-\mathrm{A} 18^{\mathrm{xiii}}$ & $93.18(3)$ \\
\hline
\end{tabular}

$\mathrm{Al3}-\mathrm{Fe} 4-\mathrm{Al} 4 \quad 112.44$ (4)

Al2-Fe4-Al11 ${ }^{\text {vii }} 131.51(5)$

$\mathrm{Al3}-\mathrm{Fe} 4-\mathrm{A} 111^{\mathrm{vii}} \quad 119.51$ (4)

Al4-Fe4-Al11 viii 86.96 (4)

$\mathrm{A} 12-\mathrm{Fe} 4-\mathrm{A} 19^{\mathrm{ii}} \quad 75.25(5)$

$\mathrm{Al}-\mathrm{Fe} 4-\mathrm{Al}^{\mathrm{ii}} \quad 136.09$ (4)

$\mathrm{A} 14-\mathrm{Fe} 4-\mathrm{A} 19^{\mathrm{ii}} \quad 111.46(4)$

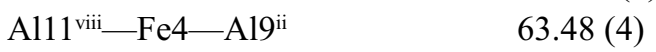

$\mathrm{A} 12-\mathrm{Fe} 4-\mathrm{Al}_{11}{ }^{\mathrm{ii}} \quad 77.16(5)$

$\mathrm{Al} 3-\mathrm{Fe} 4-\mathrm{Al13} 3^{\mathrm{ii}} \quad 132.64$ (4)

$\mathrm{A} 14-\mathrm{Fe} 4-\mathrm{Al13} 3^{\mathrm{ii}} \quad 64.73$ (4)

Al11 $1^{\text {vii_-Fe4-Al13 }}{ }^{\text {ii }} \quad 107.72(4)$

$\mathrm{A} 19^{\mathrm{ii}}-\mathrm{Fe} 4-\mathrm{Al}_{1} 3^{\mathrm{ii}} \quad 68.30$ (3)

$\mathrm{A} 12-\mathrm{Fe} 4-\mathrm{Al} 14^{\mathrm{ii}} \quad 77.54$ (5)

$\mathrm{Al3}-\mathrm{Fe} 4-\mathrm{Al14} 4^{\mathrm{ii}} \quad 70.01(4)$

Al4-Fe4-Al14ii $\quad 63.89$ (4)

Al11 $1^{\text {vii_-Fe4-Al14ii }} \quad 150.05$ (4)

$\mathrm{A} 19^{\mathrm{ii}}-\mathrm{Fe} 4-\mathrm{Al1} 4^{\mathrm{ii}} \quad 131.78$ (4)

Al13 $3^{\mathrm{ii}}-\mathrm{Fe} 4-\mathrm{A} 114^{\mathrm{ii}} \quad 67.30$ (3)

$\mathrm{A} 12-\mathrm{Fe}^{2}-\mathrm{Al}^{\mathrm{i}} \quad 77.69$ (5)

$\mathrm{Al} 3-\mathrm{Fe} 4-\mathrm{Al}^{\mathrm{i}} \quad 75.42$ (4)

Al4-Fe4-Al6 148.71 (4)

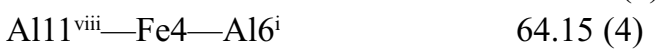

$\mathrm{A} 19^{\mathrm{ii}}-\mathrm{Fe} 4-\mathrm{Al}^{\mathrm{i}} \quad 67.56(3)$

$\mathrm{A} 113^{\mathrm{ii}}-\mathrm{Fe} 4-\mathrm{Al}^{\mathrm{i}} \quad 133.29$ (4)

$\mathrm{A} 114^{\mathrm{ii}}-\mathrm{Fe} 4-\mathrm{Al}^{\mathrm{i}} \quad 141.63$ (4)

$\mathrm{Al} 2-\mathrm{Fe} 4-\mathrm{Al}_{15} \mathrm{v} \quad 128.80(5)$

$\mathrm{Al} 3-\mathrm{Fe} 4-\mathrm{Al}_{15}^{\mathrm{v}} \quad 63.69$ (4)

$\mathrm{Al} 4-\mathrm{Fe} 4-\mathrm{A} 115^{\mathrm{v}} \quad 90.95$ (4)

Al11 ${ }^{\text {viii_-Fe4-Al15 }}{ }^{\mathrm{v}} \quad 59.11$ (4)

$\mathrm{A} 19^{\mathrm{ii}}-\mathrm{Fe} 4-\mathrm{Al}^{\mathrm{v}} \quad 116.35$ (4)

Al13 $3^{\mathrm{ii}}-\mathrm{Fe} 4-\mathrm{A} 115^{\mathrm{v}} \quad 153.92(4)$

Al14 1 - Fe4-Al15 $5^{\mathrm{v}} \quad 111.77$ (4)

$\mathrm{Al6}-\mathrm{Fe} 4-\mathrm{Al15} 5^{\mathrm{V}} \quad 64.64$ (4)

$\mathrm{A} 15-\mathrm{Fe} 5-\mathrm{Al} 6^{\mathrm{iii}} \quad 130.42(3)$

Al5-Fe5-Al6 130.42 (3)

Al6 1 iii-Fe5-Al6 98.57 (5)

$\mathrm{A} 15-\mathrm{Fe} 5-\mathrm{Al} 3^{\mathrm{iii}} \quad 71.73$ (4)

$\mathrm{Al}^{6 \mathrm{iii}}-\mathrm{Fe} 5-\mathrm{Al}{ }^{\mathrm{iii}} \quad 133.91$ (4)

$\mathrm{Al} 6-\mathrm{Fe} 5-\mathrm{Al} 3^{\mathrm{iii}} \quad 67.84$ (4)

$\mathrm{Al} 5-\mathrm{Fe} 5$ - $\mathrm{Al} 3 \quad 71.73(4)$

$\mathrm{Al6}$ iii-Fe5-Al3 67.84 (4)

Al6-Fe5-Al3 133.91 (4)

$\mathrm{Al} 3{ }^{\mathrm{iii}}-\mathrm{Fe} 5-\mathrm{Al} 3 \quad 89.87(5)$

$\mathrm{A} 15-\mathrm{Fe} 5-\mathrm{A} 17 \quad 125.88(6)$

Al6 ${ }^{\text {iii }-F e 5-A l 7 ~} \quad 71.19$ (3)

Al6-Fe5-Al7 71.18 (3)

$\mathrm{Al}{ }^{\mathrm{iii}-\mathrm{Fe} 5-\mathrm{A} 17} 134.27$ (3) 


\begin{tabular}{|c|c|}
\hline $\mathrm{Al} 6^{\mathrm{iii}}-\mathrm{Al} 7-\mathrm{Al} 8^{\mathrm{xiii}}$ & $164.56(6)$ \\
\hline $\mathrm{Fe} 1-\mathrm{Al} 7-\mathrm{A} 18$ & $54.92(3)$ \\
\hline $\mathrm{Fe} 5-\mathrm{Al} 7-\mathrm{A} 18$ & $132.33(3)$ \\
\hline $\mathrm{A} 14^{\mathrm{vi}}-\mathrm{A} 17-\mathrm{A} 18$ & $94.27(4)$ \\
\hline 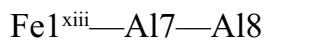 & $54.06(3)$ \\
\hline A19-Al7-Al8 & $58.81(3)$ \\
\hline $\mathrm{A} 19^{\mathrm{iii}-\mathrm{A} 17-\mathrm{A} 18}$ & $109.39(5)$ \\
\hline A16-A17-A18 & $164.56(6)$ \\
\hline $\mathrm{A} 16^{\mathrm{iii}}-\mathrm{A} 17-\mathrm{A} 18$ & $93.18(3)$ \\
\hline Al8 ${ }^{\text {xiii_-Al7-Al8 }}$ & $88.94(6)$ \\
\hline $\mathrm{Fe} 5-\mathrm{Al} 6-\mathrm{Fe}^{\mathrm{i}}$ & $141.86(5)$ \\
\hline $\mathrm{Fe} 5-\mathrm{Al} 16-\mathrm{Fe}^{\mathrm{x}}$ & $106.68(4)$ \\
\hline $\mathrm{Fe} 4^{\mathrm{i}}-\mathrm{Al} 6-\mathrm{Fe}^{\mathrm{x}}$ & $110.67(4)$ \\
\hline $\mathrm{Fe} 5-\mathrm{Al} 6-\mathrm{A} 111^{\mathrm{xi}}$ & $153.57(6)$ \\
\hline $\mathrm{Fe} 4^{\mathrm{i}}-\mathrm{Al} 6-\mathrm{Al} 11^{\mathrm{xi}}$ & $56.88(4)$ \\
\hline $\mathrm{Fe} 2^{\mathrm{x}}-\mathrm{A} 16-\mathrm{A} 111^{\mathrm{xi}}$ & $64.89(4)$ \\
\hline $\mathrm{Fe} 5-\mathrm{Al} 6-\mathrm{Al} 3^{\mathrm{iii}}$ & $56.32(3)$ \\
\hline $\mathrm{Fe} 4^{\mathrm{i}}-\mathrm{A} 16-\mathrm{A} 13^{\mathrm{iii}}$ & $137.44(5)$ \\
\hline $\mathrm{Fe} 2^{\mathrm{x}}-\mathrm{A} 16-\mathrm{A} 13^{\mathrm{iii}}$ & $64.48(3)$ \\
\hline $\mathrm{All1} 1^{\mathrm{xi}}-\mathrm{Al} 6-\mathrm{Al} 3^{\mathrm{iii}}$ & $128.18(5)$ \\
\hline $\mathrm{Fe} 5-\mathrm{Al} 6-\mathrm{Al15} 5^{\mathrm{xii}}$ & $144.22(5)$ \\
\hline $\mathrm{Fe} 4^{\mathrm{i}}-\mathrm{A} 16-\mathrm{A} 115^{\mathrm{xii}}$ & $58.49(4)$ \\
\hline $\mathrm{Fe}^{\mathrm{x}}-\mathrm{Al} 16-\mathrm{A} 115^{\mathrm{xii}}$ & $58.80(4)$ \\
\hline $\mathrm{Al} 11^{\mathrm{xi}}-\mathrm{A} 16-\mathrm{Al} 15^{\mathrm{xii}}$ & $55.08(4)$ \\
\hline $\mathrm{Al} 3^{\mathrm{iii}}-\mathrm{A} 16-\mathrm{Al} 15^{\mathrm{xii}}$ & $89.14(5)$ \\
\hline $\mathrm{Fe} 5-\mathrm{Al} 6-\mathrm{Cu} 1$ & $56.47(3)$ \\
\hline $\mathrm{Fe} 4 \mathrm{i}-\mathrm{A} 16-\mathrm{Cu} 1$ & $97.80(4)$ \\
\hline $\mathrm{Al} 11^{\mathrm{xi}}-\mathrm{Al} 1-\mathrm{Cu} 1$ & $149.96(5)$ \\
\hline $\mathrm{Al} 3{ }^{\mathrm{iii}}-\mathrm{Al} 6-\mathrm{Cu} 1$ & $58.03(3)$ \\
\hline $\mathrm{Al} 15^{\mathrm{xii}}-\mathrm{A} 16-\mathrm{Cu} 1$ & $99.33(4)$ \\
\hline $\mathrm{Fe} 5-\mathrm{Al} 6-\mathrm{A} 11$ & $56.47(3)$ \\
\hline $\mathrm{Fe} 4 \mathrm{i}-\mathrm{A} 16-\mathrm{A} 11$ & $97.80(4)$ \\
\hline $\mathrm{Fe}^{\mathrm{x}}-\mathrm{Al} 6-\mathrm{A} 11$ & $118.47(4)$ \\
\hline $\mathrm{Al11}{ }^{\mathrm{xi}}-\mathrm{A} 16-\mathrm{Al} 1$ & $149.96(5)$ \\
\hline $\mathrm{A} 13^{\mathrm{iii}}-\mathrm{A} 16-\mathrm{A} 11$ & $58.03(3)$ \\
\hline Al15 ${ }^{\mathrm{xii}}-\mathrm{A} 16-\mathrm{A} 11$ & $99.33(4)$ \\
\hline $\mathrm{Fe} 5-\mathrm{A} 16-\mathrm{A} 19^{\mathrm{iii}}$ & $113.21(5)$ \\
\hline $\mathrm{Fe} 4^{\mathrm{i}}-\mathrm{A} 16-\mathrm{A} 19^{\mathrm{iii}}$ & $55.27(3)$ \\
\hline $\mathrm{Fe} 2^{\mathrm{x}}-\mathrm{A} 16-\mathrm{A} 19^{\mathrm{iii}}$ & $116.33(5)$ \\
\hline $\mathrm{A} 111^{\mathrm{xi}}-\mathrm{A} 16-\mathrm{Al}^{\mathrm{iii}}$ & $57.12(4)$ \\
\hline $\mathrm{Al}^{3 \mathrm{ii}}-\mathrm{Al} 6-\mathrm{A} 19^{\mathrm{iii}}$ & $167.13(5)$ \\
\hline 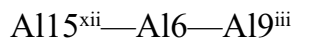 & $102.22(5)$ \\
\hline $\mathrm{A} 11-\mathrm{Al} 6-\mathrm{A} 19^{\mathrm{iii}}$ & $124.69(4)$ \\
\hline Fe5-Al6-A17 & $54.74(4)$ \\
\hline $\mathrm{Fe} 4 \mathrm{i}-\mathrm{Al} 6-\mathrm{A} 17$ & 108.88 \\
\hline $\mathrm{Fe} 2^{\mathrm{x}}-\mathrm{Al} 6-\mathrm{A} 17$ & $121.37(5)$ \\
\hline $\mathrm{A} 111^{\mathrm{xi}}-\mathrm{A} 16-\mathrm{A} 17$ & $106.48(5)$ \\
\hline $\mathrm{Al} 3{ }^{\mathrm{iii}}-\mathrm{A} 16-\mathrm{A} 17$ & $108.37(5)$ \\
\hline
\end{tabular}

\begin{tabular}{|c|c|}
\hline $\mathrm{Al} 3-\mathrm{Fe} 5-\mathrm{Al} 7$ & $134.27(3)$ \\
\hline $\mathrm{A} 15-\mathrm{Fe} 5-\mathrm{A} 11$ & $117.81(4)$ \\
\hline 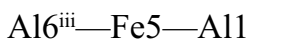 & $68.57(3)$ \\
\hline $\mathrm{Al6}-\mathrm{Fe} 5-\mathrm{Al1}$ & $68.57(3)$ \\
\hline 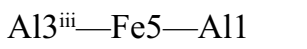 & $65.47(3)$ \\
\hline $\mathrm{A} 13-\mathrm{Fe} 5-\mathrm{A} 11$ & $65.47(3)$ \\
\hline $\mathrm{Al} 7-\mathrm{Fe} 5-\mathrm{Al} 1$ & $116.31(4)$ \\
\hline $\mathrm{A} 15-\mathrm{Fe} 5-\mathrm{Cu} 1$ & $117.81(4)$ \\
\hline 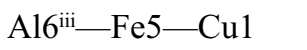 & $68.57(3)$ \\
\hline $\mathrm{Al} 6-\mathrm{Fe} 5-\mathrm{Cu} 1$ & $68.57(3)$ \\
\hline 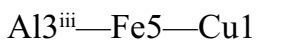 & $65.47(3)$ \\
\hline $\mathrm{A} 13-\mathrm{Fe} 5-\mathrm{Cu} 1$ & $65.47(3)$ \\
\hline $\mathrm{Al} 7-\mathrm{Fe} 5-\mathrm{Cu} 1$ & $116.31(4)$ \\
\hline $\mathrm{Al} 5-\mathrm{Fe} 5-\mathrm{Al} 14^{\mathrm{iv}}$ & $67.57(3)$ \\
\hline 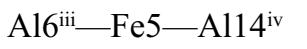 & $68.77(3)$ \\
\hline $\mathrm{Al} 6-\mathrm{Fe} 5-\mathrm{A} 114^{\mathrm{iv}}$ & $155.74(4)$ \\
\hline 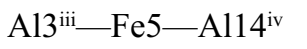 & $135.91(4)$ \\
\hline $\mathrm{Al} 3-\mathrm{Fe} 5-\mathrm{A} 114^{\mathrm{iv}}$ & $62.05(3)$ \\
\hline $\mathrm{A} 17-\mathrm{Fe} 5-\mathrm{A} 114^{\mathrm{iv}}$ & $84.94(3)$ \\
\hline $\mathrm{A} 11-\mathrm{Fe} 5-\mathrm{A} 114^{\mathrm{iv}}$ & $121.24(2)$ \\
\hline $\mathrm{A} 15-\mathrm{Fe} 5-\mathrm{A} 114^{\mathrm{x}}$ & $67.57(3)$ \\
\hline 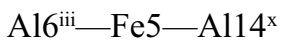 & $155.74(4)$ \\
\hline $\mathrm{A} 16-\mathrm{Fe} 5-\mathrm{Al14} 4^{\mathrm{x}}$ & $68.77(3)$ \\
\hline 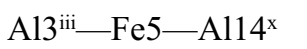 & $62.05(3)$ \\
\hline $\mathrm{Al} 3-\mathrm{Fe} 5-\mathrm{A} 114^{\mathrm{x}}$ & $135.91(4)$ \\
\hline $\mathrm{Al} 7-\mathrm{Fe} 5-\mathrm{Al1} 4^{\mathrm{x}}$ & $84.94(3)$ \\
\hline Al1-Fe5-Al14x & $121.24(2)$ \\
\hline $\mathrm{A} 114^{\mathrm{iv}}-\mathrm{Fe} 5-\mathrm{A} 114^{\mathrm{x}}$ & $114.34(5)$ \\
\hline $\mathrm{A} 15-\mathrm{Fe} 5-\mathrm{Al} 4^{\mathrm{vi}}$ & $66.44(5)$ \\
\hline $\mathrm{Al} 6^{\mathrm{iii}}-\mathrm{Fe} 5-\mathrm{Al} 4^{\mathrm{vi}}$ & $108.92(4)$ \\
\hline $\mathrm{Al} 6-\mathrm{Fe} 5-\mathrm{A} 14^{\mathrm{vi}}$ & $108.92(4)$ \\
\hline $\mathrm{A} 13^{\mathrm{iii}}-\mathrm{Fe} 5-\mathrm{A} 14^{\mathrm{vi}}$ & $117.16(4)$ \\
\hline $\mathrm{Al} 3-\mathrm{Fe} 5-\mathrm{A} 14^{\mathrm{vi}}$ & $117.17(4)$ \\
\hline $\mathrm{A} 17-\mathrm{Fe} 5-\mathrm{A} 14^{\mathrm{vi}}$ & $59.44(5)$ \\
\hline $\mathrm{Al} 1-\mathrm{Fe} 5-\mathrm{A} 14^{\mathrm{vi}}$ & $175.75(4)$ \\
\hline $\mathrm{Al} 14^{\mathrm{iv}}-\mathrm{Fe} 5-\mathrm{Al} 4^{\mathrm{vi}}$ & $59.64(3)$ \\
\hline $\mathrm{A} 114^{\mathrm{x}}-\mathrm{Fe} 5-\mathrm{Al}^{\mathrm{vi}}$ & $59.64(3)$ \\
\hline $\mathrm{A} 110-\mathrm{Fe} 1-\mathrm{Al} 7$ & $123.94(6)$ \\
\hline $\mathrm{A} 110-\mathrm{Fe} 1-\mathrm{A} 19^{\mathrm{iii}}$ & $70.63(4)$ \\
\hline $\mathrm{A} 17-\mathrm{Fe} 1-\mathrm{A} 19^{\mathrm{iii}}$ & $69.73(4)$ \\
\hline $\mathrm{A} 110-\mathrm{Fe} 1-\mathrm{A} 19$ & $70.63(4)$ \\
\hline Al7-Fe1-A19 & $69.73(4)$ \\
\hline 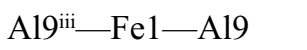 & $87.66(5)$ \\
\hline $\mathrm{A} 110-\mathrm{Fe} 1-\mathrm{A} 18^{\mathrm{xiii}}$ & $126.02(2)$ \\
\hline $\mathrm{A} 17-\mathrm{Fe} 1-\mathrm{A} 18^{\mathrm{xiii}}$ & $70.88(2)$ \\
\hline $\mathrm{A} 19^{\mathrm{iii}}-\mathrm{Fe} 1-\mathrm{A} 18^{\mathrm{xiii}}$ & $68.63(3)$ \\
\hline $\mathrm{A} 19-\mathrm{Fe} 1-\mathrm{A} 18^{\mathrm{xiii}}$ & $139.09(4)$ \\
\hline $\mathrm{A} 110-\mathrm{Fe} 1-\mathrm{A} 18$ & $126.02(2)$ \\
\hline
\end{tabular}




\begin{tabular}{|c|c|}
\hline $\mathrm{Al} 15^{\mathrm{xii}}-\mathrm{Al} 6-\mathrm{Al} 7$ & $160.87(5)$ \\
\hline $\mathrm{Cu} 1-\mathrm{Al} 6-\mathrm{Al} 7$ & $96.58(4)$ \\
\hline $\mathrm{All}-\mathrm{Al6}-\mathrm{Al} 7$ & $96.58(4)$ \\
\hline $\mathrm{A} 19^{\mathrm{iii}}-\mathrm{Al} 6-\mathrm{Al} 7$ & $59.60(4)$ \\
\hline $\mathrm{Fe} 5-\mathrm{A} 16-\mathrm{Al14} 4^{\mathrm{x}}$ & $59.39(3)$ \\
\hline $\mathrm{Fe} 4^{\mathrm{i}}-\mathrm{A} 16-\mathrm{A} 114^{\mathrm{x}}$ & $156.65(5)$ \\
\hline $\mathrm{Fe} 2^{\mathrm{x}}-\mathrm{Al} 6-\mathrm{A} 114^{\mathrm{x}}$ & $53.11(3)$ \\
\hline $\mathrm{Al11} 1^{\mathrm{xi}}-\mathrm{A} 16-\mathrm{Al} 14^{\mathrm{x}}$ & $99.81(5)$ \\
\hline $\mathrm{Al}^{\mathrm{iii}}-\mathrm{A} 16-\mathrm{A} 114^{\mathrm{x}}$ & $56.09(3)$ \\
\hline $\mathrm{Al15} 5^{\mathrm{xii}}-\mathrm{Al6}-\mathrm{Al14} 4^{\mathrm{x}}$ & $111.41(5)$ \\
\hline $\mathrm{Al1}-\mathrm{Al6}-\mathrm{Al14} 4^{\mathrm{x}}$ & $104.89(4)$ \\
\hline $\mathrm{A} 19^{\mathrm{iii}}-\mathrm{A} 16-\mathrm{A} 114^{\mathrm{x}}$ & $113.16(5)$ \\
\hline $\mathrm{Al} 7-\mathrm{Al} 6-\mathrm{Al14} 4^{\mathrm{x}}$ & $74.26(4)$ \\
\hline $\mathrm{Fe}^{\mathrm{v}}-\mathrm{A} 18-\mathrm{Fe}^{\mathrm{xiv}}$ & $71.20(5)$ \\
\hline $\mathrm{Fe} 3^{\mathrm{v}}-\mathrm{A} 18-\mathrm{Fe}^{\mathrm{xiii}}$ & $108.862(18)$ \\
\hline $\mathrm{Fe} 3^{\mathrm{xiv}}-\mathrm{A} 18-\mathrm{Fe} 1^{\mathrm{xiii}}$ & $172.76(2)$ \\
\hline $\mathrm{Fe} 3^{\mathrm{v}}-\mathrm{A} 18-\mathrm{Fe} 1$ & $172.76(2)$ \\
\hline $\mathrm{Fe}^{\mathrm{xiv}}-\mathrm{A} 18-\mathrm{Fe} 1$ & $108.861(18)$ \\
\hline 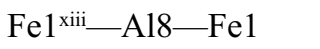 & $72.04(5)$ \\
\hline $\mathrm{Fe} 3^{\mathrm{v}}-\mathrm{A} 18-\mathrm{Al} 13^{\mathrm{iv}}$ & $56.06(3)$ \\
\hline $\mathrm{Fe}^{\mathrm{xiv}}-\mathrm{A} 18-\mathrm{A} 113^{\mathrm{iv}}$ & $113.68(5)$ \\
\hline $\mathrm{Fe}^{\mathrm{xii}}-\mathrm{A} 18-\mathrm{A} 113^{\mathrm{iv}}$ & $62.23(3)$ \\
\hline $\mathrm{Fe} 1-\mathrm{Al} 18-\mathrm{A} 113^{\mathrm{iv}}$ & $128.44(5)$ \\
\hline $\mathrm{Fe} 3^{\mathrm{v}}-\mathrm{A} 18-\mathrm{A} 113^{\mathrm{xiv}}$ & $113.68(5)$ \\
\hline $\mathrm{Fe} 3^{\mathrm{xiv}}-\mathrm{A} 18-\mathrm{Al} 13^{\mathrm{xiv}}$ & $56.06(3)$ \\
\hline $\mathrm{Fe}^{\mathrm{xiii}}-\mathrm{A} 18-\mathrm{A} 113^{\mathrm{xiv}}$ & $128.44(5)$ \\
\hline $\mathrm{Fe} 1-\mathrm{A} 18-\mathrm{A} 113^{\mathrm{xiv}}$ & $62.23(3)$ \\
\hline $\mathrm{A} 113^{\mathrm{iv}}-\mathrm{A} 18-\mathrm{A} 113^{\mathrm{xiv}}$ & $168.94(7)$ \\
\hline $\mathrm{Fe}^{\mathrm{v}}-\mathrm{A} 18-\mathrm{A} 19$ & $128.48(5)$ \\
\hline $\mathrm{Fe}^{\mathrm{xiv}}-\mathrm{A} 18-\mathrm{A} 19$ & $63.36(3)$ \\
\hline $\mathrm{Fe}^{\mathrm{xiii}}-\mathrm{A} 18-\mathrm{A} 19$ & $113.21(5)$ \\
\hline $\mathrm{Fe} 1-\mathrm{Al} 18-\mathrm{Al} 9$ & $55.45(3)$ \\
\hline $\mathrm{Al} 13^{\mathrm{iv}}-\mathrm{A} 18-\mathrm{A} 19$ & $123.90(3)$ \\
\hline $\mathrm{Al} 13^{\mathrm{xiv}}-\mathrm{A} 18-\mathrm{Al} 9$ & $57.51(3)$ \\
\hline $\mathrm{Fe}^{\mathrm{v}}-\mathrm{A} 18-\mathrm{A} 19^{\mathrm{xv}}$ & $63.36(3)$ \\
\hline $\mathrm{Fe}{ }^{\mathrm{xiv}}-\mathrm{A} 18-\mathrm{A} 19^{\mathrm{xv}}$ & $128.48(5)$ \\
\hline $\mathrm{Fe}^{\mathrm{xiii}}-\mathrm{A} 18-\mathrm{A} 19^{\mathrm{xv}}$ & $55.45(3)$ \\
\hline $\mathrm{Fe} 1-\mathrm{A} 18-\mathrm{Al}^{\mathrm{xv}}$ & $113.21(5)$ \\
\hline $\mathrm{Al}_{11} 3^{\mathrm{iv}}-\mathrm{A} 18-\mathrm{A} 19^{\mathrm{xv}}$ & $57.51(3)$ \\
\hline $\mathrm{A} 113^{\mathrm{xiv}}-\mathrm{A} 18-\mathrm{A} 19^{\mathrm{xv}}$ & $123.90(3)$ \\
\hline $\mathrm{A} 19-\mathrm{A} 18-\mathrm{A} 19^{\mathrm{xv}}$ & $167.72(7)$ \\
\hline $\mathrm{Fe}^{\mathrm{v}}-\mathrm{Al} 8-\mathrm{Al} 7^{\mathrm{xiii}}$ & $117.58(3)$ \\
\hline $\mathrm{Fe} 3^{\mathrm{xiv}}-\mathrm{A} 18-\mathrm{A} 17^{\mathrm{xiii}}$ & $132.55(3)$ \\
\hline $\mathrm{Fe} 1^{\mathrm{xiii}}-\mathrm{A} 18-\mathrm{Al} 7^{\mathrm{xiii}}$ & $54.20(4)$ \\
\hline $\mathrm{Fe} 1-\mathrm{Al} 8-\mathrm{Al} 7^{\mathrm{xiii}}$ & $56.75(4)$ \\
\hline $\mathrm{A} 113^{\mathrm{iv}}-\mathrm{A} 18-\mathrm{A} 17^{\mathrm{xiii}}$ & $107.52(4)$ \\
\hline $\mathrm{Al} 13^{\mathrm{xiv}}-\mathrm{Al} 8-\mathrm{Al} 7^{\mathrm{xiii}}$ & $80.44(4)$ \\
\hline A19-A18-A17 & $110.75(5)$ \\
\hline
\end{tabular}

\begin{tabular}{|c|c|}
\hline $\mathrm{A} 17-\mathrm{Fe} 1-\mathrm{A} 18$ & $70.88(2)$ \\
\hline $\mathrm{A} 19^{\mathrm{iii}}-\mathrm{Fe} 1-\mathrm{A} 18$ & $139.09(4)$ \\
\hline $\mathrm{A} 19-\mathrm{Fe} 1-\mathrm{A} 18$ & $68.62(3)$ \\
\hline $\mathrm{A} 18^{\mathrm{xiii}}-\mathrm{Fe} 1-\mathrm{A} 18$ & $107.96(5)$ \\
\hline $\mathrm{Al} 10-\mathrm{Fe} 1-\mathrm{Al} 7^{\mathrm{xiii}}$ & $127.08(5)$ \\
\hline $\mathrm{Al} 7-\mathrm{Fe} 1-\mathrm{Al} 7^{\mathrm{xiii}}$ & $108.99(4)$ \\
\hline $\mathrm{A} 19^{\mathrm{iii}}-\mathrm{Fe} 1-\mathrm{A} 17^{\mathrm{xiii}}$ & $135.32(3)$ \\
\hline $\mathrm{A} 19-\mathrm{Fe} 1-\mathrm{Al} 7^{\mathrm{xiii}}$ & $135.32(3)$ \\
\hline $\mathrm{A} 18^{\mathrm{xiii}}-\mathrm{Fe} 1-\mathrm{A} 17^{\mathrm{xiii}}$ & $69.19(2)$ \\
\hline $\mathrm{A} 18-\mathrm{Fe} 1-\mathrm{Al} 7^{\mathrm{xiii}}$ & $69.19(2)$ \\
\hline $\mathrm{A} 110-\mathrm{Fe} 1-\mathrm{A} 14^{\mathrm{xviii}}$ & $67.80(5)$ \\
\hline $\mathrm{Al} 7-\mathrm{Fe} 1-\mathrm{Al} 4^{\mathrm{xviii}}$ & $168.26(5)$ \\
\hline $\mathrm{A} 19^{\mathrm{iii}-\mathrm{Fe} 1-\mathrm{A} 14^{\mathrm{xviii}}}$ & $117.90(4)$ \\
\hline $\mathrm{A} 19-\mathrm{Fe} 1-\mathrm{A} 14^{\mathrm{xviii}}$ & $117.90(4)$ \\
\hline $\mathrm{A} 18^{\mathrm{xii}}-\mathrm{Fe} 1-\mathrm{A} 14^{\mathrm{xviii}}$ & $102.79(3)$ \\
\hline $\mathrm{A} 18-\mathrm{Fe} 1-\mathrm{A} 14^{\mathrm{xviii}}$ & 102.79 \\
\hline $\mathrm{Al} 7^{\mathrm{xii}}-\mathrm{Fe} 1-\mathrm{A} 14^{\mathrm{xviii}}$ & $59.28(5)$ \\
\hline $\mathrm{Al} 10-\mathrm{Fe} 1-\mathrm{A} 113^{\mathrm{xvi}}$ & $66.00(3)$ \\
\hline $\mathrm{Al} 7-\mathrm{Fe} 1-\mathrm{Al} 13^{\mathrm{xvi}}$ & $122.50(2)$ \\
\hline $\mathrm{A} 19^{\mathrm{iii}}-\mathrm{Fe} 1-\mathrm{A} 113^{\mathrm{xvi}}$ & $61.80(3)$ \\
\hline $\mathrm{A} 19-\mathrm{Fe} 1-\mathrm{A} 113^{\mathrm{xvi}}$ & $132.99(4)$ \\
\hline $\mathrm{A} 18^{\mathrm{xii}}-\mathrm{Fe} 1-\mathrm{A} 113^{\mathrm{xvi}}$ & $64.19(3)$ \\
\hline $\mathrm{A} 18-\mathrm{Fe} 1-\mathrm{A} 113^{\mathrm{xvi}}$ & $155.93(4)$ \\
\hline $\mathrm{A} 17^{\mathrm{xiii}}-\mathrm{Fe} 1-\mathrm{A} 113^{\mathrm{xvi}}$ & $86.96(3)$ \\
\hline $\mathrm{A} 14^{\mathrm{xviii}}-\mathrm{Fe} 1-\mathrm{A} 113^{\mathrm{xvi}}$ & $60.01(3)$ \\
\hline $\mathrm{Al} 10-\mathrm{Fe} 1-\mathrm{Al} 13^{\mathrm{xiv}}$ & $66.00(3)$ \\
\hline $\mathrm{A} 17-\mathrm{Fe} 1-\mathrm{A} 113^{\mathrm{xiv}}$ & $122.50(2)$ \\
\hline $\mathrm{A} 19^{\mathrm{iii}}-\mathrm{Fe} 1-\mathrm{A} 113^{\mathrm{xiv}}$ & $132.99(4)$ \\
\hline $\mathrm{A} 19-\mathrm{Fe} 1-\mathrm{A} 113^{\mathrm{xiv}}$ & $61.80(3)$ \\
\hline $\mathrm{A} 18^{\mathrm{xii}}-\mathrm{Fe} 1-\mathrm{A} 113^{\mathrm{xiv}}$ & $155.93(3)$ \\
\hline $\mathrm{A} 18-\mathrm{Fe} 1-\mathrm{A} 113^{\mathrm{xiv}}$ & $64.19(3)$ \\
\hline $\mathrm{A} 17^{\mathrm{xiii}}-\mathrm{Fe} 1-\mathrm{A} 113^{\mathrm{xiv}}$ & $86.96(3)$ \\
\hline $\mathrm{A} 14^{\mathrm{xviii}}-\mathrm{Fe} 1-\mathrm{A} 113^{\mathrm{xiv}}$ & $60.01(2)$ \\
\hline $\mathrm{Al} 13^{\mathrm{xvi}}-\mathrm{Fe} 1-\mathrm{Al} 13^{\mathrm{xiv}}$ & $112.93(5)$ \\
\hline Al10-Fe1-Fe1 $1^{\text {xiii }}$ & $179.91(5)$ \\
\hline $\mathrm{A} 17-\mathrm{Fe} 1-\mathrm{Fe} 1^{\mathrm{xiii}}$ & $56.16(4)$ \\
\hline $\mathrm{A} 19^{\mathrm{iii}}-\mathrm{Fe} 1-\mathrm{Fe} 1^{\mathrm{xiii}}$ & $109.43(3)$ \\
\hline $\mathrm{A} 19-\mathrm{Fe} 1-\mathrm{Fe} 1^{\mathrm{xiii}}$ & $109.43(3)$ \\
\hline $\mathrm{A} 18^{\mathrm{xiii}}-\mathrm{Fe} 1-\mathrm{Fe} 1^{\mathrm{xiii}}$ & $53.98(2)$ \\
\hline $\mathrm{A} 18-\mathrm{Fe} 1-\mathrm{Fe} 1^{\mathrm{xii}}$ & $53.98(2)$ \\
\hline $\mathrm{A} 17^{\mathrm{xiii}}-\mathrm{Fe} 1-\mathrm{Fe} 1^{\mathrm{xiii}}$ & $52.83(4)$ \\
\hline $\mathrm{Al} 1{ }^{\mathrm{x} v i i i}-\mathrm{Fe} 1-\mathrm{Fe} 1^{\text {xiii }}$ & $112.11(4)$ \\
\hline $\mathrm{A} 113^{\mathrm{xvi}}-\mathrm{Fe} 1-\mathrm{Fe} 1^{\mathrm{xiii}}$ & $113.96(3)$ \\
\hline $\mathrm{A} 113^{\mathrm{xiv}}-\mathrm{Fe} 1-\mathrm{Fe} 1^{\mathrm{xiii}}$ & $113.96(3)$ \\
\hline $\mathrm{Al} 10-\mathrm{Fe} 3-\mathrm{Al} 12$ & $123.53(6)$ \\
\hline Al10-Fe3-Al11 & $66.23(5)$ \\
\hline $\mathrm{A} 112-\mathrm{Fe} 3-\mathrm{Al} 11$ & $170.24(6)$ \\
\hline $\mathrm{A} 110-\mathrm{Fe} 3-\mathrm{A} 18^{\mathrm{xxiv}}$ & $125.58(2)$ \\
\hline
\end{tabular}




\begin{tabular}{|c|c|}
\hline $\mathrm{A} 19^{\mathrm{xv}}-\mathrm{A} 18-\mathrm{A} 17^{\mathrm{xiii}}$ & $59.73(4)$ \\
\hline $\mathrm{Fe} 3$ - $-\mathrm{A} 18-\mathrm{A} 17$ & $132.55(3)$ \\
\hline $\mathrm{Fe}^{\mathrm{xiv}}-\mathrm{A} 18-\mathrm{Al} 7$ & $117.58(3)$ \\
\hline $\mathrm{Fe}^{\mathrm{xiii}-\mathrm{A} 18-\mathrm{Al} 7}$ & $56.75(4)$ \\
\hline $\mathrm{Fe} 1-\mathrm{Al} 8-\mathrm{Al} 7$ & $54.20(4)$ \\
\hline $\mathrm{Al} 13^{\mathrm{iv}}-\mathrm{A} 18-\mathrm{Al} 7$ & $80.44(4)$ \\
\hline $\mathrm{Al} 13^{\mathrm{xiv}}-\mathrm{Al} 8-\mathrm{Al} 7$ & $107.52(4)$ \\
\hline $\mathrm{A} 19-\mathrm{A} 18-\mathrm{A} 17$ & $59.73(4)$ \\
\hline $\mathrm{A} 19^{\mathrm{xv}}-\mathrm{A} 18-\mathrm{A} 17$ & $110.75(5)$ \\
\hline $\mathrm{Al} 7^{\mathrm{xiii}}-\mathrm{A} 18-\mathrm{Al} 7$ & $91.06(6)$ \\
\hline $\mathrm{Fe} 3^{\mathrm{v}}-\mathrm{A} 18-\mathrm{A} 112^{\mathrm{v}}$ & $53.42(4)$ \\
\hline $\mathrm{Fe} 3^{\mathrm{xiv}}-\mathrm{A} 18-\mathrm{A} 112^{\mathrm{v}}$ & $57.72(4)$ \\
\hline $\mathrm{Fe}^{\mathrm{xiii}}-\mathrm{A} 18-\mathrm{A} 112^{\mathrm{v}}$ & $116.18(3)$ \\
\hline $\mathrm{Fe} 1-\mathrm{A} 18-\mathrm{A} 112^{\mathrm{v}}$ & $133.06(3)$ \\
\hline $\mathrm{Al} 13^{\mathrm{iv}}-\mathrm{Al} 8-\mathrm{Al} 12^{\mathrm{v}}$ & $59.29(4)$ \\
\hline $\mathrm{Al} 13^{\mathrm{xiv}}-\mathrm{A} 18-\mathrm{A} 112^{\mathrm{v}}$ & $112.13(5)$ \\
\hline $\mathrm{A} 19-\mathrm{A} 18-\mathrm{A} 112^{\mathrm{v}}$ & $81.49(4)$ \\
\hline $\mathrm{A} 19^{\mathrm{xv}}-\mathrm{A} 18-\mathrm{Al} 12^{\mathrm{v}}$ & $107.25(4)$ \\
\hline $\mathrm{Al} 7^{\mathrm{xii}}-\mathrm{Al} 8-\mathrm{A} 112^{\mathrm{v}}$ & $166.33(4)$ \\
\hline $\mathrm{Al} 7-\mathrm{A} 18-\mathrm{A} 112^{\mathrm{v}}$ & $90.10(3)$ \\
\hline $\mathrm{Fe} 3^{\mathrm{v}}-\mathrm{Al} 8-\mathrm{Al} 12^{\mathrm{xiv}}$ & $57.72(4)$ \\
\hline $\mathrm{Fe} 3^{\text {xiv }}-\mathrm{Al} 8-\mathrm{Al} 12^{\mathrm{xiv}}$ & $53.42(4)$ \\
\hline $\mathrm{Fe}^{\mathrm{xiii}}-\mathrm{Al} 8-\mathrm{Al} 12^{\mathrm{xiv}}$ & $133.06(3)$ \\
\hline $\mathrm{Fe} 1-\mathrm{A} 18-\mathrm{Al} 12^{\mathrm{xiv}}$ & $116.18(3)$ \\
\hline $\mathrm{Al}_{11} 3^{\mathrm{iv}}-\mathrm{A} 18-\mathrm{A} 112^{\mathrm{xiv}}$ & $112.13(5)$ \\
\hline $\mathrm{Al} 13^{\mathrm{xiv}}-\mathrm{A} 18-\mathrm{A} 112^{\mathrm{xiv}}$ & $59.29(4)$ \\
\hline A19-Al8-Al12 ${ }^{\mathrm{xiv}}$ & $107.25(4)$ \\
\hline $\mathrm{A} 19^{\mathrm{xv}}-\mathrm{A} 18-\mathrm{A} 112^{\mathrm{xiv}}$ & $81.49(4)$ \\
\hline $\mathrm{Al} 7^{\mathrm{xii}}-\mathrm{A} 18-\mathrm{A} 112^{\mathrm{xiv}}$ & $90.10(3)$ \\
\hline $\mathrm{Al} 7-\mathrm{Al} 8-\mathrm{Al1} 2^{\mathrm{xiv}}$ & $166.33(4)$ \\
\hline $\mathrm{Al} 12^{\mathrm{v}}-\mathrm{Al} 8-\mathrm{A} 112^{\mathrm{xiv}}$ & $91.97(6)$ \\
\hline $\mathrm{Fe} 1-\mathrm{A} 19-\mathrm{Fe} 4^{\mathrm{ii}}$ & $151.13(5)$ \\
\hline $\mathrm{Fe} 1-\mathrm{A} 19-\mathrm{A} 111^{\mathrm{xiv}}$ & $146.65(5)$ \\
\hline $\mathrm{Fe} 4^{\mathrm{ii}}-\mathrm{A} 19-\mathrm{A} 111^{\mathrm{xiv}}$ & $58.23(4)$ \\
\hline $\mathrm{Fe} 1-\mathrm{A} 19-\mathrm{A} 113^{\mathrm{xiv}}$ & $63.91(3)$ \\
\hline $\mathrm{Fe} 4^{\mathrm{ii}}-\mathrm{A} 19-\mathrm{Al} 13^{\mathrm{xiv}}$ & $136.48(5)$ \\
\hline $\mathrm{Al11}{ }^{\mathrm{xiv}}-\mathrm{A} 19-\mathrm{Al} 13^{\mathrm{xiv}}$ & $105.24(5)$ \\
\hline $\mathrm{Fe} 1-\mathrm{Al} 9-\mathrm{Fe} 3^{\mathrm{xiv}}$ & $100.39(4)$ \\
\hline $\mathrm{Fe} 4^{\mathrm{ii}}-\mathrm{A} 19-\mathrm{Fe} 3^{\text {xiv }}$ & $108.44(4)$ \\
\hline $\mathrm{Al}_{11}{ }^{\mathrm{xiv}}-\mathrm{A} 19-\mathrm{Fe}^{\mathrm{xiv}}$ & $54.24(4)$ \\
\hline $\mathrm{Al} 13^{\mathrm{xiv}}-\mathrm{A} 19-\mathrm{Fe}^{\mathrm{xiv}}$ & $54.11(3)$ \\
\hline $\mathrm{Fe} 1-\mathrm{A} 19-\mathrm{A} 18$ & $55.93(4)$ \\
\hline $\mathrm{Fe}_{4}{ }^{\mathrm{ii}}-\mathrm{A} 19-\mathrm{A} 18$ & $145.71(5)$ \\
\hline $\mathrm{A} 111^{\text {xiv }}-\mathrm{A} 19-\mathrm{A} 18$ & $90.89(5)$ \\
\hline $\mathrm{Al} 13^{\mathrm{xiv}}-\mathrm{A} 19-\mathrm{Al} 8$ & $60.99(3)$ \\
\hline $\mathrm{Fe}^{\mathrm{xiv}}-\mathrm{A} 19-\mathrm{A} 18$ & $52.90(4)$ \\
\hline $\mathrm{Fe} 1-\mathrm{A} 19-\mathrm{Al} 6^{\mathrm{iii}}$ & $115.83(5)$ \\
\hline $\mathrm{Fe} 4^{\mathrm{ii}}-\mathrm{A} 19-\mathrm{A} 16^{\mathrm{iii}}$ & $57.17(3)$ \\
\hline
\end{tabular}

\begin{tabular}{|c|c|}
\hline $\mathrm{A} 112-\mathrm{Fe} 3-\mathrm{A} 18^{\mathrm{xxiv}}$ & $72.15(2)$ \\
\hline $\mathrm{A} 111-\mathrm{Fe} 3-\mathrm{A} 18^{\mathrm{xxiv}}$ & $102.61(3)$ \\
\hline $\mathrm{A} 110-\mathrm{Fe} 3-\mathrm{A} 18^{\mathrm{xiv}}$ & $125.58(2)$ \\
\hline $\mathrm{Al} 12-\mathrm{Fe} 3-\mathrm{Al} 8^{\mathrm{xiv}}$ & $72.15(2)$ \\
\hline $\mathrm{A} 111-\mathrm{Fe} 3-\mathrm{A} 18^{\mathrm{xiv}}$ & $102.61(3)$ \\
\hline $\mathrm{A} 18^{\mathrm{xxiv}}-\mathrm{Fe} 3-\mathrm{A} 18^{\mathrm{xiv}}$ & $108.80(5)$ \\
\hline $\mathrm{A} 110-\mathrm{Fe} 3-\mathrm{A} 113^{\mathrm{iii}}$ & $71.29(4)$ \\
\hline $\mathrm{A} 112-\mathrm{Fe} 3-\mathrm{A} 113^{\mathrm{iii}}$ & $69.48(4)$ \\
\hline $\mathrm{A} 111-\mathrm{Fe} 3-\mathrm{A} 113^{\mathrm{iii}}$ & $116.75(4)$ \\
\hline $\mathrm{A} 18^{\mathrm{xxiv}}-\mathrm{Fe} 3-\mathrm{A} 113^{\mathrm{iii}}$ & $67.99(3)$ \\
\hline $\mathrm{A} 18^{\mathrm{xiv}}-\mathrm{Fe} 3-\mathrm{A} 113^{\mathrm{iii}}$ & $140.41(4)$ \\
\hline $\mathrm{Al10}-\mathrm{Fe} 3-\mathrm{Al13}$ & $71.29(4)$ \\
\hline Al12-Fe3-Al13 & $69.48(4)$ \\
\hline $\mathrm{Al11}-\mathrm{Fe} 3-\mathrm{A} 113$ & $116.75(4)$ \\
\hline $\mathrm{A} 18^{\mathrm{xxi}}-\mathrm{Fe} 3-\mathrm{Al} 13$ & $140.41(4)$ \\
\hline $\mathrm{Al} 8^{\mathrm{xiv}}-\mathrm{Fe} 3-\mathrm{A} 113$ & $67.99(3)$ \\
\hline $\mathrm{Al} 13^{\mathrm{iii}}-\mathrm{Fe} 3-\mathrm{A} 113$ & $89.56(5)$ \\
\hline $\mathrm{Al} 10-\mathrm{Fe} 3-\mathrm{Al} 12^{\mathrm{xx}}$ & $125.76(5)$ \\
\hline $\mathrm{Al} 12-\mathrm{Fe} 3-\mathrm{Al} 12^{\mathrm{xx}}$ & $110.71(4)$ \\
\hline $\mathrm{A} 111-\mathrm{Fe} 3-\mathrm{A} 112^{\mathrm{xx}}$ & $59.53(5)$ \\
\hline $\mathrm{A} 18^{\mathrm{xxiv}}-\mathrm{Fe} 3-\mathrm{A} 112^{\mathrm{xx}}$ & $69.24(2)$ \\
\hline $\mathrm{A} 18^{\mathrm{xiv}}-\mathrm{Fe} 3-\mathrm{A} 112^{\mathrm{xx}}$ & $69.24(2)$ \\
\hline $\mathrm{A} 113^{\mathrm{iii}}-\mathrm{Fe} 3-\mathrm{A} 112^{\mathrm{xx}}$ & $134.53(3)$ \\
\hline $\mathrm{A} 113-\mathrm{Fe} 3-\mathrm{A} 112^{\mathrm{xx}}$ & $134.53(3)$ \\
\hline $\mathrm{A} 110-\mathrm{Fe} 3-\mathrm{A} 19^{\mathrm{xvi}}$ & $65.51(3)$ \\
\hline $\mathrm{Al} 12-\mathrm{Fe} 3-\mathrm{A} 19^{\mathrm{xvi}}$ & $122.33(2)$ \\
\hline $\mathrm{A} 111-\mathrm{Fe} 3-\mathrm{A} 19^{\mathrm{xvi}}$ & $59.82(2)$ \\
\hline $\mathrm{A} 18^{\mathrm{xxiv}}-\mathrm{Fe} 3-\mathrm{A} 19^{\mathrm{xvi}}$ & $63.74(3)$ \\
\hline $\mathrm{A} 18^{\mathrm{xiv}}-\mathrm{Fe} 3-\mathrm{A} 19^{\mathrm{xvi}}$ & $155.76(4)$ \\
\hline $\mathrm{Al} 13^{\mathrm{iii}}-\mathrm{Fe} 3-\mathrm{A} 19^{\mathrm{xvi}}$ & $60.83(3)$ \\
\hline $\mathrm{A} 113-\mathrm{Fe} 3-\mathrm{A} 19^{\mathrm{xvi}}$ & $133.31(4)$ \\
\hline $\mathrm{A} 112^{\mathrm{xx}}-\mathrm{Fe} 3-\mathrm{A} 19^{\mathrm{xvi}}$ & $86.83(3)$ \\
\hline $\mathrm{A} 110-\mathrm{Fe} 3-\mathrm{A} 19^{\mathrm{xiv}}$ & $65.51(3)$ \\
\hline $\mathrm{Al} 12-\mathrm{Fe} 3-\mathrm{A} 19^{\mathrm{xiv}}$ & $122.33(2)$ \\
\hline $\mathrm{A} 111-\mathrm{Fe} 3-\mathrm{A} 19^{\mathrm{xiv}}$ & $59.82(2)$ \\
\hline $\mathrm{A} 18^{\mathrm{xxiv}}-\mathrm{Fe} 3-\mathrm{A} 19^{\mathrm{xiv}}$ & $155.76(4)$ \\
\hline $\mathrm{Al} 18^{\mathrm{xiv}}-\mathrm{Fe} 3-\mathrm{A} 19^{\mathrm{xiv}}$ & $63.74(3)$ \\
\hline 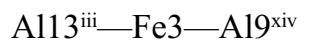 & $133.31(4)$ \\
\hline $\mathrm{Al} 13-\mathrm{Fe} 3-\mathrm{A} 19^{\mathrm{xiv}}$ & $60.83(3)$ \\
\hline $\mathrm{A} 112^{\mathrm{xx}}-\mathrm{Fe} 3-\mathrm{A} 19^{\mathrm{xiv}}$ & $86.83(3)$ \\
\hline $\mathrm{A} 19^{\mathrm{xvi}}-\mathrm{Fe} 3-\mathrm{A} 19^{\mathrm{xiv}}$ & $112.78(5)$ \\
\hline $\mathrm{A} 110-\mathrm{Fe} 3-\mathrm{Fe} 3^{\mathrm{xx}}$ & $178.25(5)$ \\
\hline $\mathrm{A} 112-\mathrm{Fe} 3-\mathrm{Fe} 3^{\mathrm{xx}}$ & $58.22(4)$ \\
\hline $\mathrm{A} 111-\mathrm{Fe} 3-\mathrm{Fe} 3^{\mathrm{xx}}$ & $112.02(5)$ \\
\hline $\mathrm{A} 18^{\mathrm{xxi}}-\mathrm{Fe} 3-\mathrm{Fe} 3^{\mathrm{xx}}$ & $54.40(2)$ \\
\hline $\mathrm{A} 18^{\mathrm{xiv}}-\mathrm{Fe} 3-\mathrm{Fe} 3^{\mathrm{xx}}$ & $54.40(2)$ \\
\hline $\mathrm{A} 113^{\mathrm{iii}}-\mathrm{Fe} 3-\mathrm{Fe} 3^{\mathrm{xx}}$ & $109.88(3)$ \\
\hline $\mathrm{A} 113-\mathrm{Fe} 3-\mathrm{Fe}^{\mathrm{xx}}$ & $109.88(3)$ \\
\hline
\end{tabular}




\begin{tabular}{|c|c|}
\hline $\mathrm{A} 111^{\mathrm{xiv}}-\mathrm{A} 19-\mathrm{Al} 6^{\mathrm{iii}}$ & $59.01(4)$ \\
\hline $\mathrm{Al} 13^{\mathrm{xiv}}-\mathrm{A} 19-\mathrm{Al} 6^{\mathrm{iii}}$ & $153.12(5)$ \\
\hline $\mathrm{Fe} 3^{\mathrm{xiv}}-\mathrm{A} 19-\mathrm{Al}^{\mathrm{iii}}$ & $102.03(4)$ \\
\hline $\mathrm{Al} 8-\mathrm{A} 19-\mathrm{Al}^{\mathrm{iii}}$ & $95.65(4)$ \\
\hline $\mathrm{Fe} 1-\mathrm{A} 19-\mathrm{Al} 7$ & $55.00(4)$ \\
\hline $\mathrm{Fe} 4^{\mathrm{ii}}-\mathrm{A} 19-\mathrm{Al} 7$ & $111.69(5)$ \\
\hline $\mathrm{Al} 11^{\mathrm{xiv}}-\mathrm{A} 19-\mathrm{Al} 7$ & $109.06(5)$ \\
\hline $\mathrm{Al} 13^{\mathrm{xiv}}-\mathrm{A} 19-\mathrm{Al} 7$ & $111.81(5)$ \\
\hline $\mathrm{Fe}^{\mathrm{xiv}}-\mathrm{A} 19-\mathrm{A} 17$ & $109.71(4)$ \\
\hline $\mathrm{A} 18-\mathrm{A} 19-\mathrm{A} 17$ & $61.47(4)$ \\
\hline $\mathrm{Al6}{ }^{\mathrm{iii}}-\mathrm{A} 19-\mathrm{Al} 7$ & $60.87(4)$ \\
\hline $\mathrm{Fe} 1-\mathrm{A} 19-\mathrm{A} 113$ & $114.71(4)$ \\
\hline $\mathrm{Fe} 4$ ii-Al9-Al13 & $56.10(3)$ \\
\hline $\mathrm{A} 111^{\mathrm{xiv}}-\mathrm{A} 19-\mathrm{A} 113$ & $96.02(5)$ \\
\hline $\mathrm{A} 113^{\text {xiv }}-\mathrm{A} 19-\mathrm{A} 113$ & $90.47(4)$ \\
\hline $\mathrm{Fe}^{\mathrm{xiv}}-\mathrm{A} 19-\mathrm{Al} 13$ & $110.70(4)$ \\
\hline A18-A19-Al13 & $151.44(4)$ \\
\hline $\mathrm{A} 16{ }^{\mathrm{iii}}-\mathrm{A} 19-\mathrm{A} 113$ & $111.67(4)$ \\
\hline A17-A19-A113 & $139.54(5)$ \\
\hline $\mathrm{Fe} 1-\mathrm{Al} 19-\mathrm{A} 110$ & $53.78(4)$ \\
\hline $\mathrm{Fe} 4$ ii-Al9-Al10 & $112.26(5)$ \\
\hline $\mathrm{A} 111^{\mathrm{xiv}}-\mathrm{A} 19-\mathrm{A} 110$ & $151.50(5)$ \\
\hline $\mathrm{A} 113^{\mathrm{xiv}}-\mathrm{A} 19-\mathrm{A} 110$ & $61.46(4)$ \\
\hline $\mathrm{Fe}^{\text {xiv }}-\mathrm{A} 19-\mathrm{A} 110$ & $115.11(4)$ \\
\hline Al8-A19-Al10 & $102.02(5)$ \\
\hline $\mathrm{Al} 66^{\mathrm{iii}}-\mathrm{A} 19-\mathrm{A} 110$ & $142.43(5)$ \\
\hline $\mathrm{Al} 7-\mathrm{A} 19-\mathrm{A} 110$ & $99.43(4)$ \\
\hline Al13-A19-Al10 & $61.11(4)$ \\
\hline $\mathrm{Fe} 1-\mathrm{A} 19-\mathrm{Al} 10^{\mathrm{xiv}}$ & $126.00(5)$ \\
\hline $\mathrm{Fe}^{\mathrm{ii}}-\mathrm{A} 19-\mathrm{A} 110^{\mathrm{xiv}}$ & $75.74(4)$ \\
\hline $\mathrm{A} 111^{\mathrm{xiv}}-\mathrm{A} 19-\mathrm{A} 110^{\mathrm{xiv}}$ & $58.55(4)$ \\
\hline $\mathrm{A} 113^{\mathrm{xiv}}-\mathrm{A} 19-\mathrm{A} 110^{\mathrm{xiv}}$ & $62.57(4)$ \\
\hline $\mathrm{Fe}^{\mathrm{xiv}}-\mathrm{A} 19-\mathrm{A} 110^{\mathrm{xiv}}$ & $51.36(3)$ \\
\hline $\mathrm{A} 18-\mathrm{A} 19-\mathrm{A} 110^{\mathrm{xiv}}$ & $101.98(5)$ \\
\hline 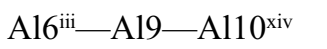 & $114.86(5)$ \\
\hline $\mathrm{Al} 7-\mathrm{A} 19-\mathrm{A} 110^{\mathrm{xiv}}$ & $160.66(5)$ \\
\hline Al13-Al9-Al10 ${ }^{\mathrm{xiv}}$ & $59.66(4)$ \\
\hline $\mathrm{A} 110-\mathrm{A} 19-\mathrm{A} 110^{\mathrm{xiv}}$ & $93.66(3)$ \\
\hline $\mathrm{Fe} 1-\mathrm{A} 19-\mathrm{Al}^{\mathrm{i}}$ & $101.02(4)$ \\
\hline $\mathrm{Fe} 4^{\mathrm{ii}}-\mathrm{A} 19-\mathrm{A} 12^{\mathrm{i}}$ & $50.12(3)$ \\
\hline $\mathrm{A} 111^{\mathrm{xiv}}-\mathrm{A} 19-\mathrm{A} 12^{\mathrm{i}}$ & $104.35(4)$ \\
\hline $\mathrm{Al} 13^{\mathrm{xiv}}-\mathrm{A} 19-\mathrm{A} 12^{\mathrm{i}}$ & $142.15(5)$ \\
\hline $\mathrm{Fe} 3^{\mathrm{xiv}}-\mathrm{A} 19-\mathrm{A} 12^{\mathrm{i}}$ & $158.17(4)$ \\
\hline $\mathrm{A} 18-\mathrm{A} 19-\mathrm{A} 12^{\mathrm{i}}$ & 141.03 \\
\hline $\mathrm{Al} 6^{\mathrm{iii}}-\mathrm{A} 19-\mathrm{Al} 2^{\mathrm{i}}$ & $64.45(4)$ \\
\hline $\mathrm{Al} 7-\mathrm{A} 19-\mathrm{Al} 2^{\mathrm{i}}$ & $79.61(4)$ \\
\hline $\mathrm{A} 113-\mathrm{A} 19-\mathrm{Al} 2^{\mathrm{i}}$ & $63.36(4)$ \\
\hline $\mathrm{A} 110-\mathrm{A} 19-\mathrm{A} 12^{\mathrm{i}}$ & $81.42(5)$ \\
\hline
\end{tabular}

$\mathrm{A} 112^{\mathrm{xx}}-\mathrm{Fe} 3-\mathrm{Fe} 3^{\mathrm{xx}}$

52.49 (4)

A19 ${ }^{\mathrm{xvi}}-\mathrm{Fe} 3-\mathrm{Fe}^{\mathrm{xx}}$

A19 ${ }^{x i v}-\mathrm{Fe} 3-\mathrm{Fe}^{\text {xx }}$

Al14iii-Fe2-Al14

Al14iii_-Fe2-Al12

Al14-Fe2-Al12

Al14iii_-Fe2-Al15

A114-Fe2-Al15

Al12-Fe2-Al15

$\mathrm{A} 114^{\mathrm{iii}}-\mathrm{Fe} 2-\mathrm{A} 15^{\mathrm{i}}$

$\mathrm{Al14}-\mathrm{Fe} 2-\mathrm{Al} 5^{\mathrm{i}}$

$\mathrm{Al12}-\mathrm{Fe} 2-\mathrm{A} 15^{\mathrm{i}}$

Al15- $\mathrm{Fe} 2-\mathrm{Al} 5^{\mathrm{i}}$

$\mathrm{A} 114^{\mathrm{iii}}-\mathrm{Fe} 2-\mathrm{Al} 6^{\mathrm{xxix}}$

Al14-Fe2-Al6 ${ }^{\text {xxix }}$

Al12-Fe2-Al6 $6^{\text {xix }}$

$\mathrm{Al15}-\mathrm{Fe} 2-\mathrm{Al} 6^{\mathrm{xxix}}$

$\mathrm{A} 15^{\mathrm{i}}-\mathrm{Fe} 2-\mathrm{A} 16^{\mathrm{xxix}}$

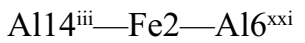

$\mathrm{A} 114-\mathrm{Fe} 2-\mathrm{A} 16^{\mathrm{xxi}}$

Al12-Fe2-Al6 ${ }^{\text {xxi }}$

Al15-Fe2-A16 ${ }^{\text {xxi }}$

$\mathrm{A} 15^{\mathrm{i}}-\mathrm{Fe} 2-\mathrm{Al}^{\mathrm{xxi}}$

$\mathrm{A} 16^{\mathrm{xxix}}-\mathrm{Fe} 2-\mathrm{A} 16^{\text {xxi }}$

Al14 ${ }^{\mathrm{iii}}-\mathrm{Fe} 2-\mathrm{A} 115^{\mathrm{xxii}}$

Al14-Fe2-Al15 ${ }^{\text {xxii }}$

$\mathrm{A} 112-\mathrm{Fe} 2-\mathrm{A} 115^{\text {xxii }}$

Al15-Fe2-Al15 ${ }^{\text {xxii }}$

$\mathrm{A} 15^{\mathrm{i}}-\mathrm{Fe} 2-\mathrm{A} 115^{\mathrm{xxii}}$

Al6 ${ }^{\text {xix }}-\mathrm{Fe} 2-\mathrm{A} 115^{\text {xxii }}$

Al6 ${ }^{\text {xxi }-F e 2-A 115 ~} 5^{\text {xxi }}$

$\mathrm{A} 114^{\mathrm{iii}}-\mathrm{Fe} 2-\mathrm{A} 111^{\mathrm{xx}}$

$\mathrm{A} 114-\mathrm{Fe} 2-\mathrm{A} 111^{\mathrm{xx}}$

$\mathrm{Al12}-\mathrm{Fe} 2-\mathrm{A} 111^{\mathrm{xx}}$

$\mathrm{Al15}-\mathrm{Fe} 2-\mathrm{A} 111^{\mathrm{xx}}$

$\mathrm{A} 15^{\mathrm{i}}-\mathrm{Fe} 2-\mathrm{A} 111^{\mathrm{xx}}$

$\mathrm{A} 16^{\mathrm{xxix}}-\mathrm{Fe} 2-\mathrm{A} 111^{\mathrm{xx}}$

$\mathrm{A} 16^{\mathrm{xxi}}-\mathrm{Fe} 2-\mathrm{A} 111^{\mathrm{xx}}$

Al15 ${ }^{\mathrm{xxi}}-\mathrm{Fe} 2-\mathrm{A} 111^{\mathrm{xx}}$

$\mathrm{Al14} 4^{\mathrm{iii}}-\mathrm{Fe} 2-\mathrm{Al} 3^{\text {xxii }}$

$\mathrm{Al14}-\mathrm{Fe} 2-\mathrm{A} 13^{\text {xxiii }}$

Al12-Fe2-Al3 ${ }^{\text {xxii }}$

Al15-Fe2-Al3 ${ }^{\text {xiii }}$

$\mathrm{Al} 5^{\mathrm{i}}-\mathrm{Fe} 2-\mathrm{Al} 3^{\text {xxiii }}$

$\mathrm{A} 16^{\text {xxix }}-\mathrm{Fe} 2-\mathrm{A} 13^{\text {xxiii }}$

$\mathrm{A} 16^{\mathrm{xxi}}-\mathrm{Fe} 2-\mathrm{A} 13^{\mathrm{xxii}}$

$\mathrm{A} 115^{\mathrm{xxi}}-\mathrm{Fe} 2-\mathrm{Al} 3^{\text {xxiii }}$

$\mathrm{A} 111^{\mathrm{xx}}-\mathrm{Fe} 2-\mathrm{A} 13^{\mathrm{xxiii}}$
113.77 (3)

113.77 (3)

$92.50(5)$

$69.30(3)$

$69.30(3)$

$116.82(3)$

$116.82(3)$

$170.01(5)$

73.17 (4)

73.17 (4)

$124.50(5)$

$65.49(5)$

$69.83(3)$

150.51 (4)

82.17 (3)

92.49 (3)

$120.56(3)$

150.52 (4)

69.83 (3)

82.17 (3)

$92.49(3)$

$120.56(3)$

$114.53(5)$

132.97 (3)

132.97 (3)

$111.05(5)$

$58.96(5)$

$124.44(5)$

$63.93(3)$

$63.93(3)$

107.31 (3)

107.31 (3)

56.22 (4)

113.79 (5)

$179.28(5)$

59.29 (3)

$59.29(3)$

54.83 (4)

131.08 (4)

59.40 (3)

123.62 (3)

59.35 (3)

61.16 (3)

149.49 (4)

60.24 (3)

89.55 (3)

$118.56(3)$ 


\begin{tabular}{|c|c|}
\hline $\mathrm{A} 110^{\mathrm{xiv}}-\mathrm{A} 19-\mathrm{A} 12^{\mathrm{i}}$ & $116.65(5)$ \\
\hline $\mathrm{Fe} 1-\mathrm{A} 110-\mathrm{Fe} 3$ & $165.79(8)$ \\
\hline Fe1-Al10-Al11 & $136.67(7)$ \\
\hline $\mathrm{Fe} 3-\mathrm{Al10}-\mathrm{Al11}$ & $57.54(4)$ \\
\hline $\mathrm{Fe} 1-\mathrm{Al} 10-\mathrm{A} 14^{\mathrm{x} v i i i}$ & $59.48(5)$ \\
\hline $\mathrm{Fe} 3-\mathrm{Al} 10-\mathrm{Al} 4^{\mathrm{xiiii}}$ & $134.73(7)$ \\
\hline $\mathrm{A} 111-\mathrm{A} 110-\mathrm{A} 14^{\mathrm{xviii}}$ & $77.19(6)$ \\
\hline $\mathrm{Fe} 1-\mathrm{A} 110-\mathrm{Al} 13^{\mathrm{xiv}}$ & $62.40(3)$ \\
\hline $\mathrm{Fe} 3-\mathrm{A} 110-\mathrm{Al13} 3^{\mathrm{xiv}}$ & $122.55(4)$ \\
\hline $\mathrm{A} 111-\mathrm{A} 110-\mathrm{A} 113^{\mathrm{xiv}}$ & $95.06(5)$ \\
\hline $\mathrm{A} 14^{\mathrm{xviii}}-\mathrm{A} 110-\mathrm{A} 113^{\mathrm{xiv}}$ & $56.79(3)$ \\
\hline $\mathrm{Fe} 1-\mathrm{Al} 10-\mathrm{Al13} 3^{\mathrm{xvi}}$ & $62.40(3)$ \\
\hline $\mathrm{Fe} 3-\mathrm{A} 110-\mathrm{Al13} 3^{\mathrm{xvi}}$ & $122.55(4)$ \\
\hline $\mathrm{A} 111-\mathrm{A} 110-\mathrm{A} 113^{\mathrm{xvi}}$ & $95.06(5)$ \\
\hline $\mathrm{A} 14^{\mathrm{xvii}}-\mathrm{A} 110-\mathrm{A} 113^{\mathrm{xvi}}$ & $56.79(3)$ \\
\hline $\mathrm{A} 113^{\mathrm{xiv}}-\mathrm{A} 110-\mathrm{A} 113^{\mathrm{xvi}}$ & $107.92(6)$ \\
\hline $\mathrm{Fe} 1-\mathrm{A} 110-\mathrm{A} 19^{\mathrm{iii}}$ & $55.59(4)$ \\
\hline $\mathrm{Fe} 3-\mathrm{Al} 10-\mathrm{A} 19^{\mathrm{iii}}$ & $114.23(5)$ \\
\hline $\mathrm{A} 111-\mathrm{A} 110-\mathrm{A} 19^{\mathrm{iii}}$ & $142.71(3)$ \\
\hline $\mathrm{A} 14^{\mathrm{xviii}}-\mathrm{A} 110-\mathrm{A} 19^{\mathrm{iii}}$ & $101.28(5)$ \\
\hline $\mathrm{A} 113^{\mathrm{xiv}}-\mathrm{A} 110-\mathrm{A} 19^{\mathrm{iii}}$ & $115.53(6)$ \\
\hline $\mathrm{A} 113^{\mathrm{xvi}}-\mathrm{A} 110-\mathrm{A} 19^{\mathrm{iii}}$ & $56.75(3)$ \\
\hline $\mathrm{Fe} 1-\mathrm{Al} 10-\mathrm{A} 19$ & $55.59(4)$ \\
\hline $\mathrm{Fe} 3-\mathrm{Al10}-\mathrm{Al} 9$ & $114.23(5)$ \\
\hline $\mathrm{A} 111$-Al10 - Al9 & $142.71(3)$ \\
\hline A14 ${ }^{\text {viii }-A 110-A 19 ~}$ & $101.28(5)$ \\
\hline $\mathrm{A} 113^{\mathrm{xiv}}-\mathrm{A} 110-\mathrm{A} 19$ & $56.75(3)$ \\
\hline Al13 xvi_Al10-Al9 & $115.53(6)$ \\
\hline $\mathrm{A} 19^{\mathrm{iii}}-\mathrm{A} 110-\mathrm{A} 19$ & $74.54(6)$ \\
\hline $\mathrm{Fe} 1-\mathrm{Al} 10-\mathrm{A} 19^{\mathrm{xvi}}$ & $121.76(4)$ \\
\hline $\mathrm{Fe} 3-\mathrm{A} 110-\mathrm{A} 19^{\mathrm{xvi}}$ & $63.13(3)$ \\
\hline $\mathrm{A} 111-\mathrm{A} 110-\mathrm{A} 19^{\mathrm{xvi}}$ & $56.91(3)$ \\
\hline $\mathrm{Al} 4^{\mathrm{xviii}}-\mathrm{A} 110-\mathrm{A} 19^{\mathrm{xvi}}$ & $93.59(4)$ \\
\hline $\mathrm{Al} 13^{\mathrm{xiv}}-\mathrm{A} 110-\mathrm{A} 19^{\mathrm{xvi}}$ & $144.87(7)$ \\
\hline $\mathrm{Al} 13^{\mathrm{xvi}}-\mathrm{A} 110-\mathrm{A} 19^{\mathrm{xvi}}$ & $59.84(3)$ \\
\hline $\mathrm{A} 19^{\mathrm{iii}}-\mathrm{A} 110-\mathrm{A} 19^{\mathrm{xvi}}$ & $86.34(3)$ \\
\hline $\mathrm{A} 19-\mathrm{A} 110-\mathrm{A} 19^{\mathrm{xvi}}$ & $157.66(6)$ \\
\hline $\mathrm{Fe} 1-\mathrm{Al} 10-\mathrm{A} 19^{\mathrm{xiv}}$ & $121.76(4)$ \\
\hline $\mathrm{Fe} 3-\mathrm{Al} 10-\mathrm{Al} 9^{\mathrm{xiv}}$ & $63.13(3)$ \\
\hline $\mathrm{A} 111-\mathrm{A} 110-\mathrm{A} 19^{\mathrm{xiv}}$ & $56.91(3)$ \\
\hline $\mathrm{A} 14^{\mathrm{xviii}}-\mathrm{A} 110-\mathrm{A} 19^{\mathrm{xiv}}$ & $93.59(4)$ \\
\hline $\mathrm{A} 113^{\mathrm{xiv}}-\mathrm{A} 110-\mathrm{A} 19^{\mathrm{xiv}}$ & $59.84(3)$ \\
\hline $\mathrm{A} 113^{\mathrm{xvi}}-\mathrm{A} 110-\mathrm{A} 19^{\mathrm{xiv}}$ & $144.87(7)$ \\
\hline $\mathrm{A} 19^{\mathrm{iii}}-\mathrm{A} 110-\mathrm{A} 19^{\mathrm{xiv}}$ & $157.66(6)$ \\
\hline $\mathrm{A} 19-\mathrm{A} 110-\mathrm{A} 19^{\mathrm{xiv}}$ & $86.34(3)$ \\
\hline $\mathrm{A} 19^{\mathrm{xvi}}-\mathrm{A} 110-\mathrm{A} 19^{\mathrm{xiv}}$ & $109.45(6)$ \\
\hline $\mathrm{Fe} 1-\mathrm{Al} 10-\mathrm{Al} 13^{\mathrm{iii}}$ & $114.78(5)$ \\
\hline $\mathrm{Fe} 3-\mathrm{Al} 10-\mathrm{Al} 13^{\mathrm{iii}}$ & $55.16(3)$ \\
\hline
\end{tabular}

$\mathrm{A} 114^{\mathrm{iii}-\mathrm{Fe} 2-\mathrm{A} 13^{\text {xxiv }}}$

$\mathrm{Al14}-\mathrm{Fe} 2-\mathrm{Al} 3^{\text {xxiv }}$

$\mathrm{Al12}-\mathrm{Fe} 2-\mathrm{Al}^{\text {xii }}$

Al15-Fe2-Al3 $3^{\text {xiv }}$

$\mathrm{A} 15^{\mathrm{i}}-\mathrm{Fe} 2-\mathrm{Al} 3^{\text {xiv }}$

$\mathrm{A} 16^{\text {xxix }}-\mathrm{Fe} 2-\mathrm{A} 13^{\text {xiv }}$

$\mathrm{A} 16^{\text {xxi }-\mathrm{Fe} 2-\mathrm{Al} 3^{\text {xxiv }}}$

Al15 ${ }^{\text {xii }-F e 2-A 13 ~}{ }^{\text {xiv }}$

Al11 ${ }^{\mathrm{xx}}-\mathrm{Fe} 2-\mathrm{Al}^{\text {xxiv }}$

$\mathrm{Al} 3^{\text {xiii }-\mathrm{Fe} 2-\mathrm{A} 13^{\text {xxiv }}}$

Al14iii-Cu2-Al14

$\mathrm{A} 114{ }^{\mathrm{iii}}-\mathrm{Cu} 2-\mathrm{A} 112$

Al14-Cu2-Al12

Al14iii-Cu2-Al15

Al14-Cu2-Al15

Al12-Cu2-Al15

$\mathrm{A} 114^{\mathrm{iii}}-\mathrm{Cu} 2-\mathrm{A} 15^{\mathrm{i}}$

$\mathrm{A} 114-\mathrm{Cu} 2-\mathrm{A} 15^{\mathrm{i}}$

$\mathrm{Al} 12-\mathrm{Cu} 2-\mathrm{Al} 5^{\mathrm{i}}$

$\mathrm{A} 115-\mathrm{Cu} 2-\mathrm{Al} 5^{\mathrm{i}}$

$\mathrm{A} 114$ iii- $\mathrm{Cu} 2-\mathrm{A} 16^{\text {xxix }}$

Al14-Cu2-A16 $6^{\text {xxix }}$

Al12-Cu2-Al6 $6^{\text {xix }}$

Al15-Cu2-Al6 ${ }^{\text {xix }}$

$\mathrm{A} 15^{\mathrm{i}}-\mathrm{Cu} 2-\mathrm{A} 16^{\text {xix }}$

$\mathrm{A} 114^{\mathrm{iii}}-\mathrm{Cu} 2-\mathrm{A} 16^{\mathrm{xxi}}$

$\mathrm{A} 114-\mathrm{Cu} 2-\mathrm{A} 16^{\mathrm{xxi}}$

$\mathrm{A} 112-\mathrm{Cu} 2-\mathrm{Al}^{\mathrm{xxi}}$

$\mathrm{A} 115-\mathrm{Cu} 2-\mathrm{Al}^{\mathrm{xxi}}$

$\mathrm{A} 15-\mathrm{Cu} 2-\mathrm{A} 16^{\mathrm{xxi}}$

$\mathrm{Al} 6^{\mathrm{xxix}}-\mathrm{Cu} 2-\mathrm{Al} 6^{\mathrm{xxi}}$

$\mathrm{A} 114^{\mathrm{iii}}-\mathrm{Cu} 2-\mathrm{A} 115^{\mathrm{xxii}}$

$\mathrm{A} 114-\mathrm{Cu} 2-\mathrm{A} 115^{\mathrm{xxi}}$

$\mathrm{A} 112-\mathrm{Cu} 2-\mathrm{A} 115^{\mathrm{xii}}$

$\mathrm{A} 115-\mathrm{Cu} 2-\mathrm{A} 115^{\mathrm{xxii}}$

$\mathrm{A} 15^{\mathrm{i}}-\mathrm{Cu} 2-\mathrm{A} 115^{\mathrm{xxii}}$

$\mathrm{A} 16^{\text {xxix }}-\mathrm{Cu} 2-\mathrm{A} 115^{\text {xxii }}$

$\mathrm{A} 16^{\mathrm{xxi}}-\mathrm{Cu} 2-\mathrm{A} 115^{\mathrm{xxii}}$

$\mathrm{A} 114^{\mathrm{iii}}-\mathrm{Cu} 2-\mathrm{A} 111^{\mathrm{xx}}$

$\mathrm{Al14}-\mathrm{Cu} 2-\mathrm{A} 111^{\mathrm{xx}}$

$\mathrm{A} 112-\mathrm{Cu} 2-\mathrm{A} 111^{\mathrm{xx}}$

$\mathrm{A} 115-\mathrm{Cu} 2-\mathrm{A} 111^{\mathrm{xx}}$

$\mathrm{A} 15^{\mathrm{i}}-\mathrm{Cu} 2-\mathrm{A} 111^{\mathrm{xx}}$

$\mathrm{A} 16^{\mathrm{xxix}}-\mathrm{Cu} 2-\mathrm{A} 111^{\mathrm{xx}}$

$\mathrm{A} 16^{\mathrm{xxi}}-\mathrm{Cu} 2-\mathrm{A} 111^{\mathrm{xx}}$

$\mathrm{A} 115^{\mathrm{xxi}}-\mathrm{Cu} 2-\mathrm{A} 111^{\mathrm{xx}}$

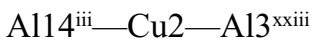

$\mathrm{Al14}-\mathrm{Cu} 2-\mathrm{A} 13^{\mathrm{xxiii}}$
$59.40(3)$

131.08 (4)

$123.62(3)$

59.35 (3)

$61.16(3)$

$60.24(3)$

149.49 (4)

89.55 (3)

$118.56(3)$

$107.65(5)$

$92.50(5)$

$69.30(3)$

$69.30(3)$

$116.82(3)$

$116.82(3)$

$170.01(5)$

73.17 (4)

73.17 (4)

124.50 (5)

$65.49(5)$

$69.83(3)$

150.51 (4)

82.17 (3)

$92.49(3)$

120.56 (3)

150.52 (4)

$69.83(3)$

82.17 (3)

92.49 (3)

$120.56(3)$

114.53 (5)

132.97 (3)

132.97 (3)

111.05 (5)

$58.96(5)$

124.44 (5)

63.93 (3)

63.93 (3)

$107.31(3)$

107.31 (3)

56.22 (4)

$113.79(5)$

179.28 (5)

59.29 (3)

$59.29(3)$

54.83 (4)

131.08 (4)

59.40 (3) 


\begin{tabular}{|c|c|c|c|}
\hline $\mathrm{Al} 11-\mathrm{Al} 10-\mathrm{Al} 13^{\mathrm{iii}}$ & $99.00(5)$ & $\mathrm{A} 112-\mathrm{Cu} 2-\mathrm{Al} 3^{\mathrm{xxiii}}$ & $123.62(3)$ \\
\hline $\mathrm{Al} 4^{\mathrm{xviii}}-\mathrm{Al} 10-\mathrm{Al} 13^{\mathrm{iii}}$ & $142.36(3)$ & $\mathrm{A} 115-\mathrm{Cu} 2-\mathrm{Al} 3^{\mathrm{xxiii}}$ & $59.35(3)$ \\
\hline $\mathrm{Al} 13^{\mathrm{xiv}}-\mathrm{Al} 10-\mathrm{Al} 13^{\mathrm{iii}}$ & $158.67(6)$ & $\mathrm{A} 15^{\mathrm{i}}-\mathrm{Cu} 2-\mathrm{A} 13^{\mathrm{xxiii}}$ & $61.16(3)$ \\
\hline $\mathrm{A} 113^{\mathrm{xvi}}-\mathrm{A} 110-\mathrm{A} 113^{\mathrm{iii}}$ & $86.81(3)$ & $\mathrm{A} 16^{\mathrm{xxix}}-\mathrm{Cu} 2-\mathrm{A} 13^{\mathrm{xxiii}}$ & $149.49(4)$ \\
\hline $\mathrm{A} 19^{\mathrm{iii}}-\mathrm{A} 110-\mathrm{A} 113^{\mathrm{iii}}$ & $59.38(4)$ & $\mathrm{A} 16^{\mathrm{xxi}}-\mathrm{Cu} 2-\mathrm{A} 13^{\mathrm{xxiii}}$ & $60.24(3)$ \\
\hline $\mathrm{A} 19-\mathrm{Al} 10-\mathrm{A} 113^{\mathrm{iii}}$ & $103.29(6)$ & $\mathrm{A} 115^{\mathrm{xxii}}-\mathrm{Cu} 2-\mathrm{A} 13^{\mathrm{xxiii}}$ & $89.55(3)$ \\
\hline $\mathrm{A} 19^{\mathrm{xvi}}-\mathrm{A} 110-\mathrm{A} 113^{\mathrm{iii}}$ & $56.00(3)$ & $\mathrm{A} 111^{\mathrm{xx}}-\mathrm{Cu} 2-\mathrm{A} 13^{\mathrm{xxiii}}$ & $118.56(3)$ \\
\hline $\mathrm{A} 19^{\mathrm{xiv}}-\mathrm{A} 110-\mathrm{A} 113^{\mathrm{iii}}$ & $115.94(6)$ & $\mathrm{A} 114^{\mathrm{iii}}-\mathrm{Cu} 2-\mathrm{A} 13^{\mathrm{xxiv}}$ & $59.40(3)$ \\
\hline $\mathrm{Fe} 1-\mathrm{A} 110-\mathrm{Al} 13$ & $114.78(5)$ & $\mathrm{Al} 14-\mathrm{Cu} 2-\mathrm{Al} 3^{\mathrm{xxiv}}$ & $131.08(4)$ \\
\hline $\mathrm{Fe} 3-\mathrm{A} 110-\mathrm{Al} 13$ & $55.16(3)$ & $\mathrm{A} 112-\mathrm{Cu} 2-\mathrm{A} 13^{\mathrm{xxiv}}$ & $123.62(3)$ \\
\hline $\mathrm{Al} 11-\mathrm{Al} 10-\mathrm{Al} 13$ & $99.00(5)$ & $\mathrm{Al} 15-\mathrm{Cu} 2-\mathrm{Al} 3^{\mathrm{xxiv}}$ & $59.35(3)$ \\
\hline $\mathrm{A} 14^{\mathrm{xviii}}-\mathrm{A} 110-\mathrm{A} 113$ & $142.36(3)$ & $\mathrm{A} 15^{\mathrm{i}}-\mathrm{Cu} 2-\mathrm{A} 13^{\mathrm{xxiv}}$ & $61.16(3)$ \\
\hline $\mathrm{A} 113^{\mathrm{xiv}}-\mathrm{A} 110-\mathrm{A} 113$ & $86.81(3)$ & $\mathrm{A} 16^{\mathrm{xxix}}-\mathrm{Cu} 2-\mathrm{A} 13^{\mathrm{xxiv}}$ & $60.24(3)$ \\
\hline $\mathrm{A} 113^{\mathrm{xvi}}-\mathrm{A} 110-\mathrm{A} 113$ & $158.67(6)$ & $\mathrm{Al} 6^{\mathrm{xxi}}-\mathrm{Cu} 2-\mathrm{Al} 3^{\mathrm{xxiv}}$ & $149.49(4)$ \\
\hline Al9 $9^{\mathrm{iii}}-\mathrm{A} 110-\mathrm{A} 113$ & $103.29(6)$ & $\mathrm{A} 115^{\mathrm{xxii}}-\mathrm{Cu} 2-\mathrm{A} 13^{\text {xxiv }}$ & $89.55(3)$ \\
\hline $\mathrm{Al} 9-\mathrm{Al10}-\mathrm{Al13}$ & $59.38(4)$ & $\mathrm{A} 111^{\mathrm{xx}}-\mathrm{Cu} 2-\mathrm{A} 13^{\mathrm{xxiv}}$ & $118.56(3)$ \\
\hline $\mathrm{A} 19^{\mathrm{xvi}}-\mathrm{A} 110-\mathrm{A} 113$ & $115.94(6)$ & $\mathrm{A} 13^{\mathrm{xxiii}}-\mathrm{Cu} 2-\mathrm{A} 13^{\mathrm{xxiv}}$ & $107.65(5)$ \\
\hline Al9 $9^{\mathrm{xiv}}-\mathrm{Al10}-\mathrm{Al13}$ & $56.00(3)$ & & \\
\hline
\end{tabular}

Symmetry codes: (i) $-x+1,-y+1,-z+1$; (ii) $-x+1, y,-z+1$; (iii) $x,-y+1, z$; (iv) $x+1 / 2,-y+3 / 2, z$; (v) $x+1 / 2, y+1 / 2, z$; (vi) $-x+3 / 2,-y+3 / 2,-z+1$; (vii) $x$, $-y+2, z$; (viii) $x+1 / 2, y+1 / 2, z+1$; (ix) $-x+1,-y+2,-z+1$; (x) $x+1 / 2, y-1 / 2, z$; (xi) $-x+1 / 2,-y+1 / 2,-z$; (xii) $-x+1 / 2,-y+1 / 2,-z+1$; (xiii) $-x+1,-y+1,-z$; (xiv) $-x+1 / 2,-y+3 / 2,-z$; (xv) $-x+1, y,-z$; (xvi) $-x+1 / 2, y-1 / 2,-z$; (xvii) $x-1 / 2,-y+3 / 2, z-1$; (xviii) $x-1 / 2, y-1 / 2, z-1$; (xix) $x, y, z-1 ;$ (xx) $-x,-y+1,-z$; (xxi) $x-1 / 2, y+1 / 2, z$; (xxii) $-x,-y+1,-z+1$; (xxiii) $x-1 / 2,-y+3 / 2, z$; (xxiv) $x-1 / 2, y-1 / 2, z$; (xxv) $-x+3 / 2, y-1 / 2,-z+1$; (xxvi) $-x+1 / 2, y+1 / 2,-z$; (xxvii) $x$, $y, z+1 ;($ xxviii) $-x+1 / 2, y+1 / 2,-z+1 ;$ (xxix) $x-1 / 2,-y+1 / 2, z$. 